Sobre a fibra especial e o teorema de RislerTeissier para filtrações

Pedro Henrique Apoliano Albuquerque Lima 


\title{
Sobre a fibra especial e o teorema de Risler-Teissier para filtrações
}

\author{
Pedro Henrique Apoliano Albuquerque Lima
}

Orientador: Prof. Dr. Victor Hugo Jorge Pérez

Tese apresentada ao Instituto de Ciências Matemáticas e de Computação - ICMC-USP, como parte dos requisitos para obtenção do título de Doutor em Ciências - Matemática. VERSÃO REVISADA.

USP - São Carlos

Abril de 2013 
Ficha catalográfica elaborada pela Biblioteca Prof. Achille Bassi e Seção Técnica de Informática, ICMC/USP, com os dados fornecidos pelo(a) autor(a)

\begin{tabular}{|c|c|}
\hline \multirow[t]{3}{*}{ A732s } & $\begin{array}{l}\text { Apoliano Albuquerque Lima, Pedro Henrique } \\
\text { Sobre a fibra especial e o teorema de Risler- } \\
\text { Teissier para filtrações / Pedro Henrique Apoliano } \\
\text { Albuquerque Lima; orientador Victor Hugo Jorge } \\
\text { Pérez. -- São Carlos, } 2013 \text {. } \\
\quad 77 \text { p. }\end{array}$ \\
\hline & $\begin{array}{l}\text { Tese (Doutorado - Programa de Pós-Graduação em } \\
\text { Matemática) -- Instituto de Ciências Matemáticas e } \\
\text { de Computação, Universidade de São Paulo, } 2013 \text {. }\end{array}$ \\
\hline & $\begin{array}{l}\text { 1. Regularidade de Castelnuovo-Mumford da fibra } \\
\text { especial. 2. Propriedade da fibra especial ser } \\
\text { Gorenstein. 3. O teorema de Risler-Teissier para } \\
\text { filtraçóes. I. Jorge Pérez, Victor Hugo, orient. II. } \\
\text { Título. }\end{array}$ \\
\hline
\end{tabular}


Os professores revelam o sentido e a meta a seus alunos. A habilidade e a força, o status e a estatura da humanidade são moldados e promovidos relativamente à qualidade e ao caráter dos seus professores. Os professores devem dedicar seu aprendizado e sua sabedoria à grande tarefa de elevar os alunos aos níveis mais altos de conhecimento e ação. As virtudes que eles ajudam a inculcar em seus alunos são essenciais para a melhoria da sociedade. O caráter é a marca de cada pessoa. Quando virtudes são profundamente enraizadas no coração, todos na sociedade podem brilhar em plena glória.

Sathya Sai Baba 



\section{Agradecimentos}

Ao meu amado Deus, Mestre e Pai, Sathya Sai Baba. "Como posso agradecer a vida, sem ser grato a você, grande tesouro da minha alma". Eu jamais teria concluído este trabalho, se não fosse a sua orientação, ajuda e principalmente o seu amor. Muito obrigado meu Swamiji.

Aos meus grandes mestres Jesus Cristo, Ganesha, Yogananda, Vana Maharaj, Narayana Maharaj e o meu caro Maharajaji. Também não esquecerei de minhas amadas mães divinas, virgem Maria e Eswarama, e a todos os outros grandes santos da humanidade.

Gostaria de agradecer a meus pais, Ana Maria Albuquerque e Luiz Gerson, por todo o amor e solidariedade que eles tem me dado desde o meu nascimento. Vocês me ajudaram muito neste doutorado, e com toda a certeza, eu jamais teria terminado este trabalho sem vocês. Amo-os muito, meus pais queridos. Eu jamais os esquecerei, o amor atravessa montanhas.

Ao meu professor, orientador e mesmo diria "pai acadêmico", Victor Hugo Jorge Pérez. Realmente o seu apoio foi decisivo neste curso. Obrigado por todo o apoio e amizade sincera que você me prestou.

Gostaria de agradecer a meus irmãos Luiz Gerson Júnior e Francisco Jander pelo o apoio moral e pelo o exemplo de dedicação ao trabalho. Também não posso esquecer de minhas cunhadas amadas e irmãs do meu coração, Samia e Laideane. Além de meus sobrinhos queridos, João Victor, Mariana e Ana Júlia, que tanto os amo.

A minha Madrinha Conceição, ou mais carinhosamente "tia Bebel", que sempre foi muito amorosa, mãe e amiga. Também aos meus padrinhos Gerson e Meire, sempre lembram de mim, muito obrigado. E também a toda a minha família maior, abrangendo tios e primos. Saudades de vocês.

A minha amada amiga Jaqueline Godoy Mesquita, que realmente foi decisiva na minha vida. Palavras são realmente muito pouco para agradecer o que você fez por mim. Também agradeço a família dela, Maria Aparecida, Marcos, Cynthia, Nicolas, Luis Mesquita, tia Patrícia 
e os irmãos Lucas e Alice, por sempre me acolherem e me ajudarem nos momentos de alegria e dificuldade. Em especial, a "dona querida", Maria Aparecida, por sempre ter sido tão doce, dedicada e torcedora do meu sucesso. Momentos difíceis muito passamos, mas o amor de Baba foi maior.

Ao meu querido amigo David Lopes, que sempre me apoiou nos momentos mais difíceis. Um dia veio a São Carlos somente para visitar-me. Muito obrigado meu irmão.

A minha grande amiga Iris de Oliveira, que sempre se demonstrou muito companheira e preocupada com meu bem-estar. Me ajudou tanto, muito obrigado.

Gostaria de agradecer de coração, e tão profundamente, aos meus amados amigos Michelângelo e Lucas, que sempre me ajudaram e me apoiaram. Como poderei retribuir?

Agradeço ao meu amigo muito sincero, Renato Alejandro, que sempre me ajudou e me apoiou. Muito obrigado meu caro amigo.

Aos meus grandes amigos e colegas acadêmicos, Poya, Masoud, Matheus Bortolan, Eder Rítis e Luis Renato que sempre me prestaram todo o tipo de ajuda. Em especial, agradeço ao Paulo, por sempre ter demonstrado ser muito compreensivo com a minha ignorância no latex. Enfim, a todos os meus amigos acadêmicos que de forma direta ou indireta contribuíram na minha jornada de trabalho.

Aos professores Raimundo Nonato, Alexandre Fernandes, Miguel Frasson, Maria Aparecida Ruas, Daniel Levcovitz, Eduardo Tengan, Alexandre Nolasco, Márcia Federson, Ali Tahzibi, Orizide Manzoli, Hildebrando Munhoz, Marcelo Saia, Roberta Wik e a todos os outros, sempre muito companheiros e amigos.

Agradeço também aos professores A. V. Jayanthan e R. Nanduri por ajudar-me em minha dúvidas.

A todos os funcionários da secretaria de pós-graduação, tais como Ana Paula e Laura, sempre tão prestativas e atenciosas.

A todos os funcionários do ICMC.

A CAPES e ao CNPq pelo apoio financeiro. 


\section{Resumo}

Seja $(R, \mathfrak{m})$ um anel Noetheriano local e $R \supset I_{1} \supset I_{2} \supset \cdots$ uma filtração de ideais de $R$. Podemos então construir a álgebra graduada $F(\mathfrak{F}):=\oplus_{n \geq 0} I_{n} / \mathfrak{m} I_{n}$, chamada de fibra especial. Esta tese objetiva a pesquisa deste anel. Investigamos sobre a sua propriedade de ser Gorenstein e a sua regularidade de Castelnuovo-Mumford. Outro objetivo, é generalizarmos o teorema de Risler-Teissier (sobre multiplicidades mistas) para o caso de filtrações de Hilbert. 



\section{Abstract}

Let $(R, \mathfrak{m})$ be a Noetherian local ring and $R \supset I_{1} \supset I_{2} \supset \cdots$ a filtration of ideals in $R$. We may then construct the graded algebra $F(\mathfrak{F}):=\oplus_{n \geq 0} I_{n} / \mathfrak{m} I_{n}$, which is called fiber cone. This thesis has the goal to research about this graded ring. We investigate its Gorenstein property and its Castelnuovo-Mumford regularity. Another aim is to generalize the Risler-Teissier's theorem (about mixed multiplicities) for the case of Hilbert filtration. 



\section{Sumário}

1 Preliminares 1

1.1 Boa filtração: propriedades e exemplos . . . . . . . . . . . . . . . . . 1

1.2 O módulo *canônico de um anel graduado . . . . . . . . . . . . . . . . . . 7

1.3 Regularidade de Castelnuovo-Mumford . . . . . . . . . . . . . . . . . . 9

2 Regularidade da álgebra de Rees e do anel graduado associado 15

3 Regularidade da fibra especial para boas filtrações 21

4 A Propriedade da fibra especial ser Gorenstein para o caso de filtrações 35

4.1 Descrição do módulo canônico da fibra especial . . . . . . . . . . . . . . . . 36

4.2 A propriedade da fibra especial de dimensão um ser Cohen-Macaulay . . . . . 40

4.3 A propriedade da fibra especial ser Gorenstein . . . . . . . . . . . . . . . . . 45

5 O Teorema de Risler-Teissier para boas filtrações

$\begin{array}{ll}\text { Apêndice } & 61\end{array}$

A Extensão analítica e cohomologia local 63

A.1 Extensão analítica . . . . . . . . . . . . . . . . . . . . . . 63

A.2 Cohomologia Local . . . . . . . . . . . . . . . . . . 65 



\section{Introdução}

Seja $(R, \mathfrak{m})$ um anel Noetheriano local e $R \supset I \supset I^{2} \supset \cdots$ a filtração $I$-adica. Temos assim, importantes álgebras graduadas como a álgebra de Rees $R(I):=\oplus_{n \geq 0} I^{n} t^{n}$, o anel graduado associado $G(I):=\oplus_{n \geq 0} I^{n} / I^{n+1}$ e a fibra especial $F(I):=\oplus_{n \geq 0} I^{n} / \mathfrak{m} I^{n}=R(I) / \mathfrak{m} R(I)$. Estas álgebras podem ser naturalmente generalizadas para o caso de uma filtração de ideais em geral, ou seja, $\mathfrak{F}: R \supset I_{1} \supset I_{2} \supset \cdots$, onde $I_{i}$ são ideais de $R$. Esta tese possui três objetivos. Estudar a regularidade de Castelnuovo-Mumford da fibra especial e a sua propriedade ser Gorenstein. O terceiro e último, é mostrar o teorema de Risler-Teissier [Te] para o caso de filtrações de Hilbert.

Nos artigos [CZ], [CZ2] e [CZ3], Cortadellas e Zarzuela, estudaram a profundidade da fibra especial fazendo uso de certos módulos graduados associados a filtrações de módulos. Jayanthan e Nanduri, em [JN], usaram alguns resultados desses artigos para estudar a regularidade (de Castelnuovo-Mumford) da fibra especial.

A regularidade da álgebra de Rees e do anel graduado associado são bem conhecidas, como pode ser verificado nos trabalhos [HZ], [O], [H], [T], [JU], etc. No artigo [HZ], por exemplo, Hoa e Zarzuela obtêm muitos resultados entre o número de redução e os $a$-invariantes de boas filtrações. Ooishi, em [O], prova que a regularidade de uma álgebra de Rees e do anel graduado associado de um ideal $I$ são iguais. Esta fórmula foi também descoberta por Johnson e Ulrich em [JU]. Depois, em [T], Trung mostrou que existe uma relação entre os $a$-invariantes da álgebra de Rees e do anel graduado associado, e então eles provaram a igualdade $\operatorname{reg} F(I)=\operatorname{reg} G(I)$. Foi possível mostrar no presente trabalho a mesma igualdade para o caso de boas filtrações.

No caso $I$-adico, Jayanthan e Nanduri em [JN] encontram, sob certas condições, relações entre a regularidade da fibra especial e a regularidade do anel graduado associado. Além disso, eles obtêm que de fato a igualdade entre elas pode ocorrer. Daremos neste trabalho uma teoria análoga sobre a regularidade da fibra especial, da álgebra de Rees e do anel graduado associado para o caso de boas filtrações. Mostramos que para este último caso, a regularidade da fibra 
especial comporta-se similarmente ao caso $I$-adico. Temos também encontrado outros resultados em que a igualdade $\operatorname{reg} F(\mathfrak{F})=\operatorname{reg} G(\mathfrak{F})$ é verdadeira.

Com relação ao segundo objetivo, nos perguntamos quando as álgebras acima citadas são Gorenstein ou Cohen-Macaulay, e outras de suas propriedades. Esta resposta é dada em diversos artigos, como por exemplo [HRZ], [GN], [HZ], [TVZ], [I], [HKU1], [HKU2] ou [JPV]. Em [HRZ], é determinado, sob certas classes de ideais, o expoente $n \geq 0$ para o qual $R\left(I^{n}\right)$ e $G\left(I^{n}\right)$ sejam Gorenstein. Goto e Nishida investigam em [GN], a propriedade da álgebra de Rees simbólica ser Cohen-Macaulay ou Gorenstein com respeito a ideais primos de dimensão um em anéis locais de Cohen-Macaulay, e então eles generalizam para o caso mais geral da álgebra de Rees $R(\mathfrak{F})$ e o anel graduado associado $G(\mathfrak{F})$ com respeito a filtrações gerais $\mathfrak{F}$ de ideais em anéis locais Noetherianos arbitrários. Além disso, em [HZ], Zarzuela e Hoa dão algumas consequências que generalizam resultados bem conhecidos no caso de filtrações $I$-adicas. Por exemplo, eles mostram, sob certas condições, que a propriedade de $R(\mathfrak{F})$ ser Gorenstein, onde $\mathfrak{F}$ é uma boa filtração, implica na propriedade de $G(\mathfrak{F})$ ser Gorenstein. Trung, Viêt e Zarzuela, em [TVZ], estendem os resultados de Ikeda em [I] para álgebra de Rees de filtrações. No artigo [HKU1], Heinzer, Kim e Ulrich dão um critério, em termos de ideais, para que o anel graduado $G(I)$ seja Gorenstein. Depois, eles generalizaram em [HKU2] estes mesmos resultados para o caso de filtrações de Hilbert. Jayanthan, Puthenpurakal e Verma, em [JPV], estudam a propriedade de $F(I)$ ser Gorenstein para ideais m-primários $I$ de multiplicidade quase minimal.

Neste trabalho, estamos interessados na propriedade da fibra especial ser Gorenstein para o caso de filtrações. No artigo [JN], Jayanthan e Nanduri, encontram (para o caso I-adico) descrições, em termos de ideais, para o módulo canônico da fibra especial. Eles também mostram que se ambos, o anel graduado associado e a fibra especial forem anéis de Cohen-Macaulay, então as regularidades dos seus módulos canônicos são iguais. Além disso, quando $F(I)$ é Cohen-Macaulay e $G(I)$ é Gorenstein, eles dão um critério para dizer se a fibra especial é Gorenstein. Um dos nossos objetivos neste trabalho é dar uma teoria análoga sobre a propriedade da fibra especial ser Gorenstein no caso de filtrações em geral. Nós mostramos que esses resultados podem ser generalizados para o caso de filtrações de Hilbert. Vale ressaltar que as multiplicidades mistras são ferramentas muito importantes para o estudo da propriedade da fibra especial ser um anel de Gorenstein, como pode ser visto por exemplo no artigo [JPV]. Neste, 
os autores obtêm resultados sob o caso de filtrações adicas. Inicialmente tinhamos a intenção de generalizar este artigo para o caso de filtrações de Hilbert. No entanto, vimos que para esse intento ainda se fazia necessário a generalização, para o caso de filtrações de Hilbert, de alguns resultados clássicos, como por exemplo, o teorema de Rees [Sw] e também o teorema de RislerTeissier [Te]. Dessa forma, para a conclusão de nosso trabalho, fizemos um estudo a respeito das multiplicidades mistas afim de verificar que o teorema de Risler-Teissier, que diz respeito a essas multiplicidades, permanece ainda válido para o caso de filtrações de Hilbert.

As multiplicidades mistas tiveram início em 1957 com o artigo de Bhattacharya [Ba], onde ele descreveu polinômios de Hilbert para dois ideais e os seus coeficientes de maior grau. O nome multiplicidades mistas apareceu primeiramente no artigo de Teissier [Te], publicado em 1973, no qual ele e Risler estudaram, usando elementos superficiais, o polinômio de Hilbert e seus coeficientes, agora para um número finito qualquer de ideais. Teissier mostra em [Te] por exemplo, que em um anel Noetheriano local $(R, \mathfrak{m})$ com ideais $I_{1}, \ldots, I_{k} \mathfrak{m}$-primários, as multiplicidades mistas com respeito a tais ideais são um inteiro positivo. Além disso, ele mostra que essas multiplicidades são iguais a multiplicidade de um ideal gerado por um sequência superficial com respeito aos ideais citados.

Agora fazemos um cronograma dos principais resultados deste trabalho. No Capítulo 1 damos uma teoria básica necessária para o bom estendimento dos resultados principais, tais como as definições e propriedades elementares de boa filtração e redução minimal. Também damos as principais propriedades do módulo canônico de um anel graduado e de sua regularidade de Castelnuovo-Mumford. No capítulo 2, tratamos das relações entre os $a$-invariantes cohomológicos do anel graduado associado e da álgebra de Rees, e depois mostramos no Corolário 2.4 que a regularidade dos mesmos são iguais no caso de boas filtrações. No capítulo 3, mostramos no Teorema 3.3, que se a extensão analítica é um, então a regularidade da fibra especial é limitada superiormente pela regularidade do anel graduado associado. Temos generalizado esse resultado mostrando que se a profundidade depth $F(\mathfrak{F})$ é limitada inferiormente por $s-1$, onde $s$ é a extensão analítica de $\mathfrak{F}$, então $\operatorname{reg} F(\mathfrak{F}) \leq \operatorname{reg} G(\mathfrak{F})$. Mostramos também no Teorema 3.10, que no caso em que a extensão analítica $s$ é arbitrária, pode ocorrer também que a regularidade da fibra especial seja limitada inferiormente pela regularidade do anel graduado associado. Então, se além disso, limitarmos inferiormente a profundidade da fibra especial por 
$s-1$, então teremos a igualdade entre as regularidades. No capítulo 4, para o caso de filtrações de Hilbert, damos na Proposição 4.1, uma descrição para o módulo canônico da fibra especial e também mostramos uma nova versão para a caracterização da sua propriedade de ser Gorenstein, como pode ser visto no Teorema 4.13. Finalmente, no capítulo 5, definimos as multiplicidades mistas com respeito a uma quantidade finita de filtrações de Hilbert, e depois mostramos que o teorema de Risler-Teissier permanece ainda válido neste caso; ver Teorema 5.12. 


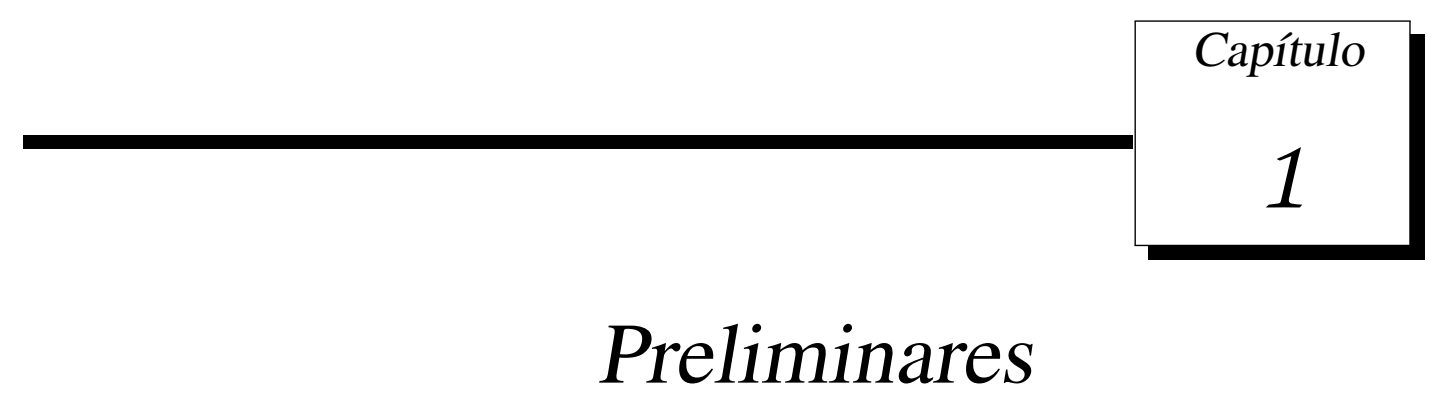

Como preliminares, introduzimos ao leitor os conceitos básicos que serão utilizados no desenrolar deste trabalho, tais como a definição de diferentes tipos de filtrações e suas propriedades. Necessitaremos também de uma pequena revisão das propriedades de um módulo canônico graduado. Finalmente, definimos a regularidade de Castelnuovo-Mumford e falamos um pouco sobre sua aplicabilidade geométrica.

Durante este trabalho, sempre supomos que todos os anéis em questão são comutativos e com unidade.

\subsection{Boa filtração: propriedades e exemplos}

Começemos com a definição dos três tipos de filtração que serão tratados nesse trabalho.

Definição 1.1. Seja $R$ um anel e $I$ um ideal de $R$. Uma sequência $\mathfrak{F}=\left(I_{n}\right)_{n \geq 0}$ de ideais de $R$ é chamada uma filtração de $R$ se $I_{0}=R \supset I_{1} \supseteq I_{2} \supseteq I_{3} \supseteq \cdots, I_{1} \neq R$ e $I_{i} I_{j} \subseteq I_{i+j}$ para todo $i, j \geq 0$. Além disso, dizemos que $\mathfrak{F}$ é uma $I$-boa filtração se $I I_{i} \subseteq I_{i+1}$ para todo $i \geq 0$ e $I_{n+1}=I I_{n}$ para todo inteiro positivo $n$ suficientemente grande. Uma filtração $\mathfrak{F}$ é chamada uma boa filtração se ela é uma $I$-boa filtração para algum ideal $I$ de $R$. Observe que $\mathfrak{F}$ é uma boa filtração se e somente se ela é uma $I_{1}$-boa filtração. Uma filtração $\mathfrak{F}$ que seja $I_{1}$-boa e tal que $I_{1}$ é m-primário, é chamada de filtração de Hilbert .

Comentário 1.2. Por [B, Corolário III.3.1.4], F é uma I-boa filtração se e somente se $I I_{i} \subseteq I_{i+1}$ para todo $i \geq 0$ e se existe um inteiro $r$ tal que $I_{n} \subseteq I^{n-r}$ para todo $n$ (onde $I_{n}=I^{n}:=R$ para $n \leq 0)$. 
Exemplo 1.3. A I-adica filtração $\mathfrak{F}=\left(I^{n}\right)_{n \geq 0}$ é um exemplo trivial de boas filtrações.

Existem diversos exemplos de boas filtrações que não sejam $I$-adicas. Vejamos algumas bastante conhecidas.

Exemplo 1.4. Seja $I$ um ideal de um anel Noetheriano $R$ que contenha um elemento não divisor de zero. Ratliff e Rush, em [RR], introduziram o seguinte ideal associado a $I$ :

$$
\widetilde{I}=\bigcup_{i \geq 1}\left(I^{i+1}: I^{i}\right)
$$

chamado o ideal de Ratliff-Rush associado a I (ou fecho de Ratliff-Rush). Ele é o maior ideal tendo a propriedade que $(\widetilde{I})^{n}=I^{n}$ para todo $n$ suficientemente grande, como pode ser visto em [RR, Teorema 2.1]. É fácil de mostrar que para cada inteiro positivo $k$,

$$
\widetilde{I^{k}}=\bigcup_{i \geq 1}\left(I^{i+k}: I^{i}\right)
$$

Além disso, por [RR, Comentário 2.3.2], $\widetilde{I^{k}}=I^{k}$ para $k$ suficientemente grande. Portanto $\mathfrak{F}=$ $\left(\widetilde{I_{n}}\right)_{n \geq 0}$ é uma $I$-boa filtração. Em particular, se $R$ é local com maximal $\mathfrak{m}$ e $I$ é um ideal $\mathfrak{m}$ primário, então $\mathfrak{F}=\left(\widetilde{I_{n}}\right)_{n \geq 0}$ é uma filtração de Hilbert.

Antes de dar início ao próximo exemplo relembremos a definição de função e polinômio de Hilbert-Samuel. Se $\mathfrak{F}$ é uma filtração de Hilbert num anel Noetheriano local $(R, \mathfrak{m})$ de dimensão $d$, a função $H_{\mathfrak{F}}(n)=\ell\left(R / I_{n}\right)$ é chamada a função de Hilbert-Samuel de $\mathfrak{F}$. Para $n$ suficientemente grande, $\ell\left(R / I_{n}\right)$ é um polinômio $P_{\mathfrak{F}}(n)$ de grau $d$, chamado o polinômio de Hilbert-Samuel $d e \mathfrak{F}$. Como no caso de filtrações adicas, existem inteiros únicos $e_{0}(\mathfrak{F}), \ldots, e_{d}(\mathfrak{F})$, chamados coeficientes de Hilbert de $\mathfrak{F}$, tais que

$$
P_{\mathfrak{F}}(n)=\sum_{i=0}^{d}(-1)^{i} e_{i}(\mathfrak{F})\left(\begin{array}{c}
n+d-1-i \\
d-i
\end{array}\right)
$$

para $n \in \mathbb{Z}$. O coeficiente líder $e_{0}(\mathfrak{F})$ é um inteiro positivo chamado de multiplicidade (de Hilbert-Samuel) de $\mathfrak{F}$. Usaremos a notação $e(I)=e_{R}(I)=e_{0}(I)$, onde $I$ é um ideal m-primário de $R$, para denotar a multiplicidade de Hilbert-Samuel da filtração $I$-adica. 
Exemplo 1.5. Seja $R$ um anel Noetheriano local de dimensão $d$ com ideal maximal $\mathfrak{m}$ e $I$ um ideal m-primário. Se $R$ é formalmente equidimensional, então o fecho integral de $I$, denotado por $\bar{I}$, é o único e maior ideal contendo $I$ para o qual $e_{0}(I)=e_{0}(\bar{I})$; ver [NR]. Já é conhecido que $R$ é um anel analiticamente não-ramificado se e somente se para todo ideal $I \subseteq \mathfrak{m}$, exista um inteiro $k=k(I)$ tal que $\overline{I^{n}} \subseteq I^{n-k}$; ver por exemplo [R, Teorema 5.32]. Logo, pelo Comentário $1.2,\left(\overline{I^{n}}\right)_{n \geq 0}$ é uma $I$-boa filtração se e somente se $R$ é analiticamente não-ramificado.

O ideal de Ratliff-Rush $\widetilde{I}$ de $I$ é o único e maior ideal contendo $I$ tal que $e_{i}(I)=e_{i}(\widetilde{I})$ para todo $0 \leq i \leq d(\operatorname{ver}[\mathrm{RR}$, Teorema 2.1]).

Mais geralmente, se $R$ é formalmente equidimensional e tal que $R / \mathfrak{m}$ seja um corpo infinito, é mostrado em [S, Teorema 1] que para cada inteiro $0 \leq k \leq d$, existe um único e maior ideal $I_{\{k\}}$ contendo $I$ e contido no fecho integral $\bar{I}$ de $I$ com a propriedade que

$$
e_{i}\left(I_{\{k\}}\right)=e_{i}(I) \text { para } 0 \leq i \leq k
$$

Dessa forma, temos uma cadeia de ideais

$$
I=I_{\{d+1\}} \subseteq I_{\{d\}} \subseteq \cdots \subseteq I_{\{1\}} \subseteq I_{\{0\}}=\bar{I}
$$

Para cada $k$, o ideal $I_{\{k\}}$ é chamado o $k$-ésimo ideal coeficiente de (ou $e_{k}$-ideal associado a) $I$. É claro que se $I$ contém um elemento regular, $I_{\{d\}}$ é o fecho de Ratliff-Rush de $I$.

Agora seja $\mathfrak{F}_{k}:=\left(\left(I^{n}\right)_{\{k\}}\right)_{n \geq 0}$ para cada $k \in\{0,1, \ldots, d+1\}$. Então temos uma cadeia de filtrações

$$
\mathfrak{F}_{d+1} \subseteq \mathfrak{F}_{d} \subseteq \cdots \subseteq \mathfrak{F}_{1} \subseteq \mathfrak{F}_{0}
$$

onde $\mathfrak{F}_{d+1}=\left(I^{n}\right)_{n \geq 0}$ é a filtração $I$-adica e $\mathfrak{F}_{0}=\left(\overline{I^{n}}\right)_{n \geq 0}$ é a filtração dada pelo fecho integral das potências de $I$. Além disso, se $I$ contém um elemento não divisor de zero, então $\mathfrak{F}_{d}$ é a filtração dada por ideais de Ratliff-Rush associado a potências de $I$. Quando $k=1$, $\mathfrak{F}_{1}$ é chamada de filtração obtida pelo fecho $e_{1}$. Se em adição, $R$ é analiticamente não-ramificado, então para cada $k$, a filtração $\mathfrak{F}_{k}$ é $I$-boa.

Além do fecho integral de um ideal, temos um outro importante fecho, chamado fecho tight. Este último pode ser usado, por exemplo, para provar o Teorema de Briançon-Skoda sobre fecho 
integral de ideais em anéis regulares. Além disso, o fecho tight também é usado para provar várias conjecturas na teoria de homologia.

Exemplo 1.6. Seja $R$ um anel Noetheriano de característica prima $p>0$. Seja $R^{o}=R \backslash \bigcup\{\mathfrak{p} \mid \mathfrak{p} \in$ $\operatorname{Min}(R)\}$, onde $\operatorname{Min}(R)$ denota o conjunto de ideais primos minimais de $R$. Então, se $I$ é um ideal de $R$, o fecho tight de I é definido como sendo o conjunto

$$
I^{*}:=\left\{x \in R \mid \exists c \in R^{o}, \text { para o qual } c x^{q} \in I^{[q]} \text { para todo } q \text { suficientemente grande }\right\}
$$

onde $I^{[q]}$ denota o ideal gerado pelas $q$-ésimas potências de todos os elementos de $I$. Em adição, se $R$ é um anel local de maximal $\mathfrak{m}$ contendo um corpo que seja analiticamente não-ramificado e formalmente equidimensional, então $\left(\left(I^{n}\right)^{*}\right)_{n \geq 0}$ é uma $I$-boa filtração. Se em adição, $I$ é um ideal m-primário, então $\left(\left(I^{n}\right)^{*}\right)_{n \geq 0}$ é uma filtração de Hilbert.

Seja $(R, \mathfrak{m})$ um anel local Noetheriano. Dada qualquer filtração $\mathfrak{F}$, podemos construir os seguintes anéis graduados

$$
\begin{gathered}
R(\mathfrak{F})=R \oplus I_{1} t \oplus I_{2} t^{2} \oplus \cdots, R^{\prime}(\mathfrak{F})=\bigoplus_{n \in \mathbb{Z}} I_{n} t^{n} \subseteq R\left[t, t^{-1}\right], \\
G(\mathfrak{F})=R^{\prime}(\mathfrak{F}) /\left(t^{-1}\right) R^{\prime}(\mathfrak{F})=R / I_{1} \oplus I_{1} / I_{2} \oplus I_{2} / I_{3} \oplus \cdots .
\end{gathered}
$$

Chamamos $R(\mathfrak{F})$ de álgebra de Rees com respeito a $\mathfrak{F}, R^{\prime}(\mathfrak{F})$ de álgebra estendida de Rees com respeito a $\mathfrak{F}$ e $G(\mathfrak{F})$ o anel graduado associado com respeito a $\mathfrak{F}$. Denotamos também o ideal irrelevante de $G(\mathfrak{F})$ por $G(\mathfrak{F})_{+}=\oplus_{n \geq 1} I_{n} / I_{n+1}$. Se $\mathfrak{F}$ é uma filtração $I$-adica, isto é, $\mathfrak{F}=\left(I^{n}\right)_{n \geq 0}$ para algum ideal $I$, denotamos a álgebra de Rees $R(\mathfrak{F})$ por $R(I)$ e o anel graduado associado $G(\mathfrak{F})$ por $G(I)$. Uma filtração $\mathfrak{F}$ é chamada Noetheriana se $R(\mathfrak{F})$ é um anel Noetheriano. Uma boa filtração é uma filtração Noetheriana. Filtrações Noetherianas satisfazem $\cap_{n \geq 0} I_{n}=0$. Adaptando a prova de [M, Teorema 15.7], pode-se mostrar que se $\mathfrak{F}$ é Noetheriana, então $\operatorname{dim} G(\mathfrak{F})=\operatorname{dim} R$. Geralmente denotamos $\operatorname{depth} G(\mathfrak{F})$ para $\operatorname{depth}_{\mathfrak{M}} G(\mathfrak{F})$, onde $\mathfrak{M}=\mathfrak{m} / I_{1} \oplus I_{1} / I_{2} \oplus I_{2} / I_{3} \cdots$ é o único ideal maximal homogêneo de $G(\mathfrak{F})$. Se $\mathfrak{F}$ é uma filtração de Hilbert, então $G(\mathfrak{F})$ é um $G\left(I_{1}\right)$-módulo finito.

Para qualquer filtração $\mathfrak{F}=\left(I_{n}\right)_{n \geq 0}$ e qualquer ideal $J$ de $R$, denotamos $\mathfrak{F} / J$ como sendo a filtração $\left(\left(I_{n}+J\right) / J\right)_{n \geq 0}$ no anel $R / J$. Além disso, se $\mathfrak{F}$ é uma filtração Noetheriana (resp. de 
Hilbert) então $\mathfrak{F} / J$ é uma filtração Noetheriana (resp. de Hilbert).

Uma reducão de uma filtração $\mathfrak{F}$ é um ideal $J \subseteq I_{1}$ tal que $J I_{n}=I_{n+1}$ para $n$ suficientemente grande. Também sabemos que $J \subseteq I_{1}$ é uma redução de $\mathfrak{F}$ se e somente se $R(\mathfrak{F})$ é um $R\left(I_{1}\right)$ módulo finito. Por [B, Teorema III.3.1.1 e Corolário III.3.1.4], $R(\mathfrak{F})$ é um $R\left(I_{1}\right)$-módulo finito se e somente se existe um inteiro $k$ tal que $I_{n} \subseteq\left(I_{1}\right)^{n-k}$ para todo $n$. Uma redução minimal de $\mathfrak{F}$ é uma redução de $\mathfrak{F}$ que seja minimal com respeito a inclusão. Por [HZ, Proposição 2.6], têm-se que reduções minimais de $\mathfrak{F}$ sempre existem. Uma boa filtração $\mathfrak{F}$ é chamada equimúltipla se $I_{1}$ é equimúltipla, isto é, $s\left(I_{1}\right)=$ ht $I_{1}$, onde $s\left(I_{1}\right)=\operatorname{dim} F\left(I_{1}\right)$. Filtrações de Hilbert são exemplos de filtrações equimúltiplas.

Se $J$ é uma redução da filtração $I$-adica, dizemos simplesmente que $J$ é uma redução de $I$. Por [NR], reduções minimais de ideais sempre existem. Se $R(\mathfrak{F})$ é um $R\left(I_{1}\right)$-módulo, então $J$ é uma redução de $\mathfrak{F}$ se e somente se $J$ é uma redução de $I_{1}$. Logo, se $R / \mathfrak{m}$ é infinito, reduções minimais de filtrações de Hilbert sempre existem e são geradas por $\operatorname{dim} R$ elementos. Para reduções minimais $J$ de $\mathfrak{F}$, primeiramente definimos $r_{J}(\mathfrak{F}):=\min \left\{n \in \mathbb{Z} \mid I_{n}=J I_{n-1}\right\}$. O número de Redução de $\mathfrak{F}$ é definido como sendo $r(\mathfrak{F})=\min \left\{r_{J}(\mathfrak{F}) \mid J\right.$ é uma redução minimal de $\left.\mathfrak{F}\right\}$.

Seja $\mathfrak{F}$ uma filtração Noetheriana. Para qualquer elemento $x \in I_{1}$, denotamos $x^{*}$ como sendo a imagem de $x$ em $G(\mathfrak{F})_{1}=I_{1} / I_{2}$ e $x^{o}$ como sendo a imagem de $x$ em $F(\mathfrak{F})_{1}=I_{1} / \mathfrak{m} I_{1}$. Se $x^{*}$ é um elemento regular de $G(\mathfrak{F})$, então $x$ é um elemento regular de $R$ e por [HZ, Lema 3.4], $G(\mathfrak{F} /(x)) \cong G(\mathfrak{F}) /\left(x^{*}\right)$.

Definição 1.7. Um elemento $x \in I_{1}$ é chamado superficial para $\mathfrak{F}$ se existe um inteiro $c$ tal que $\left(I_{n+1}: x\right) \cap I_{c}=I_{n}$ para todo $n \geq c$. Uma sequência $x_{1}, \ldots, x_{k}$ é chamada uma sequência superficial para $\mathfrak{F}$ se $x_{1}$ é um elemento superficial para $\mathfrak{F}$ e $x_{i}$ é um elemento superficial para $\mathfrak{F} /\left(x_{1}, \ldots, x_{i-1}\right)$, onde $2 \leq i \leq k$.

Definição 1.8. Dado um elemento $x \in I_{1}$, sua imagem $x^{o} \in G(\mathfrak{F})$ é dita ser superficial (ou filterregular) para $G(\mathfrak{F})$ se $x \in I_{1}$ é um elemento superficial para $\mathfrak{F}$.

Em geral, se $f_{1}, \ldots, f_{r}$ é uma sequência de elementos homogêneos de uma álgebra graduada Noetheriana $S=\oplus_{n \geq 0} S_{n}$ sobre um anel $S_{0}$, ela é chamada uma sequência filter-regular de $S$, se $f_{i} \notin \mathfrak{p}$ para todos os primos $\mathfrak{p} \in \operatorname{Ass}\left(S /\left(f_{1}, \ldots, f_{i-1}\right)\right)$ tais que $S_{+} \nsubseteq \mathfrak{p}, i=1, \ldots, r$.

Proposição 1.9. [HZ, Comentário 2.10] Um elemento $x \in R$ é superficial para $\mathfrak{F}$ se e somente 
se $\left(0:_{G(\mathfrak{F})} x^{*}\right)_{n}=0$ para todo $n$ suficientemente grande, e equivalentemente se $x^{*} \notin \mathfrak{p}$ para qualquer ideal primo homogêneo $\mathfrak{p} \in \operatorname{Ass} G(\mathfrak{F})$ tal que $G(\mathfrak{F})_{+} \nsubseteq \mathfrak{p}$.

Demonstração. Se $x \in I_{1}$ é um elemento superficial para $\mathfrak{F}$, existe um inteiro $c$ satisfazendo $\left(I_{n+1}: x\right) \cap I_{c}=I_{n}$, para todo $n \geq c$. Assim, para $n \geq c,\left(I_{n+2}: x\right) \cap I_{n} \subseteq\left(I_{n+2}: x\right) \cap I_{c}=I_{n+1}$. Isso significa que $\left(0:_{G(\mathfrak{F})} x^{*}\right)_{n}=0$ para $n \geq c$.

Definição 1.10. Dado um elemento $x \in I_{1}$, a imagem $x^{o}$ é dita ser superficial (ou filter-regular) para $F(\mathfrak{F})$ se existe $c>0$ tal que $\left(\mathfrak{m} I_{n+1}: x\right) \cap I_{n}=\mathfrak{m} I_{n}$ para todo $n>c$.

É fácil mostrar que $x^{o}$ é superficial em $F(\mathfrak{F})$ se e somente se $\left(0:_{F(\mathfrak{F})} x^{*}\right)_{n}=0$ para todo $n$ suficientemente grande.

Lema 1.11. Seja $(R, \mathfrak{m})$ um anel local e $\mathfrak{F}$ uma boa filtração. Se a $\in I_{1}$ então a imagem $a^{o} \in$ $I_{1} / \mathfrak{m} I_{1}$ em $F(\mathfrak{F})$ é um não divisor de zero em $F(\mathfrak{F})$ se e somente se $\left(\mathfrak{m} I_{n+1}: a\right) \cap I_{n}=\mathfrak{m} I_{n}$ para todo $n \geq 0$.

Demonstração. Se $b \in\left(\mathfrak{m} I_{n+1}: a\right) \cap I_{n}$ então $b a \in \mathfrak{m} I_{n+1}$, de modo que $\left(b+\mathfrak{m} I_{n}\right) a^{o}=0$. Se $a^{o}$ é um não divisor de zero em $F(\mathfrak{F})$ então $b+\mathfrak{m} I_{n}=0$, isto é, $b \in \mathfrak{m} I_{n}$.

Agora, suponha $\left(\mathfrak{m} I_{n+1}: a\right) \cap I_{n}=\mathfrak{m} I_{n}$ para todo $n \geq 0$. Seja $b \in I_{n}$ tal que $\left(b+\mathfrak{m} I_{n}\right) a^{o}=0$. Então $b a \in \mathfrak{m} I_{n+1}$, de modo que $b \in\left(\mathfrak{m} I_{n+1}: a\right) \cap I_{n}=\mathfrak{m} I_{n}$. Portanto $b+\mathfrak{m} I_{n}=0$.

Proposição 1.12. [HM, p. 67] Seja $\mathfrak{F}$ uma filtração Noetheriana e assuma que grade $I_{1} \geq k$. Se $x_{1}, \ldots, x_{k}$ é uma sequência superficial para $\mathfrak{F}$, então $x_{1}, \ldots, x_{k}$ é uma sequência regular.

Demonstração. Por indução sobre $k$, é suficiente provar o caso $k=1$. Suponhamos que $u x=0$, onde $x \in I_{1}$. Existe um inteiro positivo $c$ tal que para $n \geq c$, temos $\left(I_{1}\right)^{c} u \subseteq \cap_{n \geq c}\left(\left(I_{n+1}: x\right) \cap I_{c}\right)=$ $\cap_{n \geq c} I_{n}=0$. Assim $u=0$.

Proposição 1.13. [HM, Lema 2.1] Seja $\mathfrak{F}$ uma filtração Noetheriana e $x_{1}, \ldots, x_{k}$ uma sequência superficial para $\mathfrak{F}$. Se $\operatorname{grade} G(\mathfrak{F})_{+} \geq k$, então $x_{1}^{*}, \ldots, x_{k}^{*}$ é uma sequência regular.

Demonstração. É suficiente provar o caso $k=1$. Se $y \in\left(0: x_{1}^{*}\right)$ é homogêneo então $y G(\mathfrak{F})_{+}^{n}=$ 0 para $n$ suficientemente grande (desde que $x_{1}$ é superficial). Usando [BS, Teorema 6.2.7], obtemos

$$
\left(0: x_{1}^{*}\right) \subseteq H_{G(\mathfrak{F})_{+}}^{0}(G(\mathfrak{F}))=0 .
$$


Um outra prova para a Proposição acima pode ser encontrada apartir de [P, Teorema 8].

O próximo resultado é um análogo da proposição anterior e por esta razão a prova será omitida.

Proposição 1.14. Sejam $\mathfrak{F}$ uma filtração Noetheriana e $x_{1}, \ldots, x_{k}$ uma sequência superficial para F. Se $\operatorname{depth} F(\mathfrak{F}) \geq k$ então $x_{1}^{o}, \ldots, x_{k}^{o}$ é uma sequência regular.

\subsection{O módulo *anônico de um anel graduado}

A maior parte dos objetos associados a uma variedade diferenciável suave e a uma variedade algébrica suave são seus fibrados tangentes e cotangentes, e os seus vários tensores derivados. O fibrado canônico, bastante conhecido em geometria algébrica, por exemplo, é a maior potência exterior do fibrado cotangente. Além disso, se a variedade é afim, então as seções do fibrado canônico formam um módulo sobre o anel de coordenadas da variedade algébrica chamada de módulo canônico. Nesta seção, apresentamos as propriedades algébricas básicas do módulo canônico.

Seja $R$ um anel Noetheriano, $M$ um $R$-módulo finito e $\mathfrak{p} \in \operatorname{Spec}(R)$. O número (finito) $\mu_{i}(\mathfrak{p}, M)=\operatorname{dim}_{k(\mathfrak{p})} \operatorname{Ext}_{R_{\mathfrak{p}}}^{i}\left(k(\mathfrak{p}), M_{\mathfrak{p}}\right)=\operatorname{dim}_{k(\mathfrak{p})}\left(\operatorname{Ext}_{R}^{i}(R / \mathfrak{p}, M)\right)_{\mathfrak{p}}$ é chamado o i-ésimo número de Bass de $M$ com respeito a $\mathfrak{p}$, onde $k(\mathfrak{p})=R_{\mathfrak{p}} / \mathfrak{p} R_{\mathfrak{p}}$ é o corpo residual do anel local $R_{\mathfrak{p}}$.

Definição 1.15. Seja $(R, \mathfrak{m})$ um anel local de Cohen-Macaulay de dimensão $d$. Um $R$-módulo finito $C$ é um módulo canônico de $R$ se

$$
\mu_{i}(\mathfrak{m}, C)= \begin{cases}0 & \text { se } i \neq d \\ 1 & \text { se } i=d\end{cases}
$$

Logo $C$ é um módulo canônico de $R$ se e somente se

$$
\operatorname{Ext}_{R}^{i}(R / \mathfrak{m}, C) \cong\left\{\begin{array}{cc}
0 & \text { para } i \neq d \\
R / \mathfrak{m} & \text { para } i=d
\end{array}\right.
$$

Isto é uma motivação para a definição de módulo *canônico sobre um anel *local de CohenMacaulay, onde * relata que estamos trabalhando em termos homogêneos. Primeiramente dare- 
mos a definição de anel *local.

Definição 1.16. Seja $R$ um anel graduado. Um ideal graduado $\mathfrak{m}$ de $R$ é chamado * maximal, se todo ideal graduado contendo propriamente $\mathfrak{m}$ for igual a $R$. Dizemos que $R$ é ${ }^{*}$ local se ele tem um único ideal $\mathfrak{m}$ *maximal. Um anel ${ }^{*}$ local com ideal *maximal $\mathfrak{m}$ será denotado por $(R, \mathfrak{m})$.

De forma similar ao caso usual, podemos definir a dimensão no caso homogêneo de um anel graduado.

Definição 1.17. Se $(R, \mathfrak{m})$ é * local, definimos a altura de $\mathfrak{m}$ como sendo a definição de *dimensão de $R$, denotada por * $\operatorname{dim} R$.

Essa * dimensão pode ser caracterizada, já que a *dimensão de $R$ é igual ao supremo de todos os números $h$ para os quais exista uma cadeia de ideais primos graduados $\mathfrak{p}_{0} \subset \mathfrak{p}_{1} \subset \cdots \subset \mathfrak{p}_{h}$ em $R$; ver [BH, Teorema 1.58].

Agora podemos definir o módulo *canônico sobre um anel *local de Cohen-Macaulay.

Se $(R, \mathfrak{m})$ é um anel *local de Cohen-Macaulay de *dimensão $d$, um $R$-módulo finito graduado $\omega_{R}$ é dito ser um módulo ${ }^{*}$ canônico de $R$ se existem isomorfismos homogêneous

$$
{ }^{*} \operatorname{Ext}_{R}^{i}\left(R / \mathfrak{m}, \omega_{R}\right) \cong\left\{\begin{array}{cc}
0 & \text { para } i \neq d \\
R / \mathfrak{m} & \text { para } i=d
\end{array}\right.
$$

Observação 1.18. O módulo * canônico $\omega_{R}$ de um anel * local $R$ é também um módulo canônico de $R$ e é unicamente determinado a menos de isomorfismos homogêneos; ver [BH, Proposição 3.6.9].

Exemplo 1.19. Sejam $R=k\left[x_{1}, \ldots, x_{n}\right]$ o anel dos polinômios sobre o corpo $k$, onde $k=R / m$ é o corpo residual de $R$. Seja $\mathfrak{m}=\left(x_{1}, \ldots, x_{n}\right)$ o ideal maximal graduado de $R$. É bem conhecido que o complexo de Koszul de $x_{1}, \ldots, x_{n}$ dá uma resolução minimal graduada livre de $k$ da forma

$$
0 \rightarrow R(-n) \rightarrow R(-n+1)^{\left(\begin{array}{c}
n \\
n-1
\end{array}\right)} \rightarrow \cdots \rightarrow R(-2)^{\left(\begin{array}{c}
n \\
2
\end{array}\right)} \rightarrow R(-1)^{\left(\begin{array}{l}
n \\
1
\end{array}\right)} \rightarrow R \rightarrow k \rightarrow 0 .
$$

Daí concluímos que ${ }^{*} \operatorname{Ext}_{R}^{i}(R / \mathfrak{m}, R)=0$ para $i \neq n$ e ${ }^{*} \operatorname{Ext}_{R}^{n}(R / \mathfrak{m}, R)=(R / \mathfrak{m})(n)$. Dessa forma, o módulo * canônico de $R$ é $R(-n)$. 
Definição 1.20. Seja $(R, \mathfrak{m})$ um anel *local positivamente graduado de Cohen-Macaulay com módulo *anônico $\omega_{R}$. Define-se o a-invariante de $R$ como sendo

$$
a(R):=-\min \left\{n \in \mathbb{Z}:\left(\omega_{R}\right)_{n} \neq 0\right\}
$$

É também possível mostrar que $a(R)=\sup \left\{n:\left[H_{\mathfrak{m}}^{d}(R)\right]_{n} \neq 0\right\}$, onde $d$ é a ${ }^{*}$ dimensão de $R$ (ver [BS, Definição e Comentários 13.4.7]).

Agora, relatamos alguns resultados bastante conhecidos a respeito do módulo *canônico. Por exemplo, $R$ é Gorenstein se e somente se $\omega_{R} \cong R(q)$ para algum inteiro $q \in \mathbb{Z}$, como pode ser visto em [BH, Proposição 3.6.11]. Se $R$ é Gorenstein, este $q$ é único e tal que $a(R)=q$. Além disso, por [BH, Proposição 3.6.12], se $(R, \mathfrak{m}) \rightarrow(S, \mathfrak{n})$ é um homomorfismo finito de anéis * locais de Cohen-Macaulay, então $\omega_{S} \cong \operatorname{Ext}_{R}^{r}\left(S, \omega_{R}\right)$, onde $r={ }^{*} \operatorname{dim} R-{ }^{*} \operatorname{dim} S$. É também conhecido (ver por exemplo [HIO, Corolário 36.11]) que se $R$ é um anel graduado arbitrário definido sobre um anel local, então $\omega_{R}$ é um $R$-módulo graduado de Cohen-Macaulay de dimensão *injetiva finita e tal que depth $\omega_{R}=\operatorname{dim} R$. Para ver a definição de dimensão *injetiva, referenciamos o livro de Bruns e Herzog [BH].

\subsection{Regularidade de Castelnuovo-Mumford}

Seja $R=\bigoplus_{n \geq 0} R_{n}$ um anel graduado finitamente gerado sobre um anel Noetheriano $R_{0}$ por elementos de grau um. Para qualquer $R$-módulo graduado $M$, denotamos por $M_{n}$, a componente homogênea de grau $n$ de $M$ e seja

$$
a(M):= \begin{cases}\max \left\{n \mid M_{n} \neq 0\right\} & \text { se } M \neq 0 \\ -\infty & \text { se } M=0\end{cases}
$$

Seja $R_{+}$o ideal gerado pelos elementos homogêneous de grau positivo de $R$. Para $i \geq 0$ e qualquer $R$-módulo graduado $M$, seja

$$
a_{i}(M):=a\left(H_{R_{+}}^{i}(M)\right)
$$

onde $H_{R_{+}}^{i}(M)$ denota o também chamado $i$-ésimo módulo de cohomologia local de $M$ com respeito ao ideal irrelevante $R_{+}$(ver apêndice). A regularidade de Castelnuovo-Mumford (ou 
simplesmente regularidade) de $M$ é definida como sendo o número

$$
\operatorname{reg}(M):=\max \left\{a_{i}(M)+i \mid i \geq 0\right\}
$$

Além disso, a regularidade de Castelnuovo-Mumford (ou simplesmente regularidade) $\operatorname{reg}^{l}(M)$ de $M$ no nível $l$ é definida por

$$
\operatorname{reg}^{l}(M):=\max \left\{a_{i}(M)+i \mid i \geq l\right\}
$$

É claro que $\operatorname{reg}^{0}(M)=\operatorname{reg}(M)$ e $\operatorname{reg}^{l}(M) \leq \operatorname{reg}^{l-1}(M) \leq \ldots \leq \operatorname{reg}^{0}(M)=\operatorname{reg}(M)$. Além disso, desde que $H_{R_{+}}^{i}(M(t))=\left(H_{R_{+}}^{i}(M)\right)(t)\left(\operatorname{ver}\left[\mathrm{BS}\right.\right.$, Comentário 13.1.9]), temos $\operatorname{reg}^{l}(M(t))=\operatorname{reg}^{l}(M)-$ $t$ para todo $t \in \mathbb{Z}$. Para o caso em que $M=R$, a regularidade reg $R$ é um importante invariante do anel graduado $R$ (ver [EG] e [O]).

Proposição 1.21. Assuma que $R=\bigoplus_{n \geq 0} R_{n}$ é positivamente graduado e seja

$$
0 \rightarrow L \rightarrow M \rightarrow N \rightarrow 0
$$

uma sequência exata de R-módulos graduados finitos e homomorfismo homogêneous. Mostre que

(1) $\operatorname{reg}(L) \leq \max \{\operatorname{reg}(M), \operatorname{reg}(N)+1\}$;

(2) $\operatorname{reg}^{l}(M) \leq \max \left\{\operatorname{reg}^{l}(L), \operatorname{reg}^{l}(N)\right\}$;

(3) $\operatorname{reg}^{l}(N) \leq \max \left\{\operatorname{reg}^{l+1}(L)-1, \operatorname{reg}^{l}(M)\right\}$. Em particular, $\operatorname{reg}(N) \leq \max \{\operatorname{reg}(L)-1, \operatorname{reg}(M)\}$.

Demonstração. Segue fazendo uso do Comentário A.13 (ver apêndice).

Embora este trabalho dê importância à parte algébrica da regularidade de CastelnuovoMumford, também gostaríamos de relatar alguns resultados que testificam sua importância geométrica. Para ver mais detalhes referenciamos [BS, Capítulo 14] e sugerimos [H] para a teoria básica a respeito de geometria algébrica.

Seja $k$ um corpo algebricamente fechado e $r \in \mathbb{N}$. Denotemos $\mathbb{A}^{r+1}(k)$ como sendo o $(r+1)$ espaço afim sobre $k$, ou seja, os elementos da forma $\left(c_{0}, \ldots, c_{r}\right)$, tal que $c_{i} \in k$ para $0 \leq i \leq r$. 
Também definimos o conjunto

$$
\left\{\left(c_{0}: \cdots: c_{r}\right):\left(c_{0}, \ldots, c_{r}\right) \in \mathbb{A}^{r+1}(k) \backslash\{0\}\right\} / \sim,
$$

onde $\left(c_{0}: \cdots: c_{r}\right) \sim\left(c_{0}^{\prime}: \cdots: c_{r}^{\prime}\right)$ se somente se existe $\lambda \neq 0$ tal que $c_{i}=\lambda c_{i}^{\prime}$ para todo $i$. Este conjunto é denotado por $\mathbb{P}^{r}(k)$ e chamado de $r$-espaço projetivo sobre $k$. Este conjunto é dotado com a topologia de Zariski, ou seja, seus conjuntos fechados, chamados de conjuntos algébricos projetivos, são da forma

$$
V_{\mathbb{P}^{r}(k)}(I):=\left\{\left(c_{0}: \cdots: c_{r}\right) \in \mathbb{P}^{r}(k): f\left(c_{0}, \ldots, c_{r}\right)=0 \text { para todo } f \in I\right\},
$$

onde $I$ é um ideal homogêneo próprio de $k\left[X_{0}, \ldots X_{r}\right]$. É claro que $I$ é sempre finitamente gerado. Um subconjunto fechado irredutível de $\mathbb{P}^{r}(k)$ é chamado uma variedade projetiva. Além disso, um subconjunto aberto não-vazio de uma variedade projetiva é chamado de variedade quasiprojetiva.

Dado um subconjunto fechado $V=V_{\mathbb{P}^{r}(k)}(I) \subseteq \mathbb{P}^{r}(k)$, o cone afim sobre $V$, denotado por Cone $(V) \subseteq \mathbb{A}^{r+1}(k)$, é definido como sendo

$$
\operatorname{Cone}(V)=\left\{\left(c_{0}, \ldots, c_{r}\right) \in \mathbb{A}^{r+1}(k) \backslash\{0\}:\left(c_{0}: \cdots: c_{r}\right) \in V\right\} \cup\{0\}=V_{\mathbb{A}^{r+1}(k)}(I),
$$

ao qual é um cone algébrico afim em $\mathbb{A}^{r+1}(k)$, ou seja, um subconjunto fechado $V(J) \subseteq \mathbb{A}^{r+1}(k)$ tal que $J \subseteq k\left[x_{0}, \ldots, x_{r}\right]$ é um ideal homogêneo.

Definição 1.22. Seja $V \subset \mathbb{P}^{r}(k)$ uma variedade projetiva. Seja $I_{\mathbb{P}^{r}(k)}(V):=I_{\mathbb{A}^{r+1}(k)}(\operatorname{Cone}(V))$ o ideal primo homogêneo de $k\left[X_{0}, \ldots, X_{r}\right]$. A regularidade de Castelnuovo-Mumford de $V$, denotada por reg $(V)$, é definida como sendo

$$
\operatorname{reg}(V):=\operatorname{reg}\left(I_{\mathbb{P}^{r}(k)}(V)\right) .
$$

Seja $d \in \mathbb{N}$. Considere a aplicação

$$
\begin{aligned}
\varphi: \mathbb{P}^{1}(k) & \longrightarrow \mathbb{P}^{d}(k) \\
(x: y) & \longmapsto\left(x^{d}: x^{d-1} y: \cdots: x y^{d-1}, y^{d}\right) .
\end{aligned}
$$


A imagem $\varphi\left(\mathbb{P}^{1}(k)\right)$ é chamada a curva racional normal no d-espaço projetivo, e denotada por $C^{d}$. Se $d=3, C^{3}$ é também chamada cúbica retorcida.

Agora tome

$$
\mathscr{I}=\mathscr{I}_{r}^{d}=\left\{\left(v_{0}, \ldots, v_{r}\right) \in \mathbb{Z}^{r+1}: v_{i} \geq 0 \text { e } v_{0}+\ldots+v_{r}=d\right\}
$$

Considere a aplicação

$$
\begin{aligned}
\alpha: k\left[Y_{v}: v \in \mathscr{I}\right] & \longrightarrow k\left[X_{0}, \ldots X_{r}\right] \\
Y_{\left(v_{0}, \ldots, v_{r}\right)} & \longmapsto X_{0}^{v_{0}} \ldots X_{r}^{v_{r}},
\end{aligned}
$$

onde $k\left[Y_{v}: v \in \mathscr{I}\right]$ é o anel dos polinômios nas variáveis independentes $\left(Y_{v}\right)_{v \in \mathscr{I}}$ sobre o corpo $k$. A imagem $\alpha\left(k\left[Y_{v}: v \in \mathscr{I}\right]\right)$, denotada por $k\left[X_{0}, \ldots X_{r}\right]^{(d)}$, é chamada o $d$-ésimo subanel Veroneseano de $k\left[X_{0}, \ldots X_{r}\right]$.

O seguinte resultado dá uma aplicação da regularidade de Castelnuovo-Mumford à geometria algébrica.

Comentário 1.23. [BS, Exercício 1.23] Seja $\phi: k\left[Y_{0}, \ldots, Y_{d}\right] \longrightarrow k\left[X_{0}, X_{1}\right]^{(d)}$ um homomorfismo de k-álgebras definido por $\phi\left(Y_{0}\right)=X_{0}^{d}, \phi\left(Y_{1}\right)=X_{0}^{d-1} X_{1}, \ldots, \phi\left(Y_{d-1}\right)=X_{0} X_{1}^{d-1}$ e $\phi\left(Y_{d}\right)=X_{1}^{d}$. $\operatorname{Logo} \operatorname{Ker} \phi=I_{\mathbb{P}^{d}(k)}\left(C^{d}\right)$.

(1) Calculando-se reg $\left(C^{d}\right)$ pode-se concluir que $I_{\mathbb{P}^{d}(k)}\left(C^{d}\right)$ pode ser gerado por quadráticas;

(2) Calcula-se então a dimensão da componente de grau 2 de $I_{\mathbb{P}^{d}(k)}\left(C^{d}\right)$, como um espaço vetorial sobre $k$;

(3) Pode-se concluir que $I_{\mathbb{P d}_{(k)}}\left(C^{d}\right)$ pode ser gerado pelos determinantes das matrizes menores $2 \times 2$ da matriz

$$
\left(\begin{array}{cccccc}
Y_{0} & Y_{1} & Y_{2} & \ldots & Y_{d-2} & Y_{d-1} \\
Y_{1} & Y_{2} & Y_{3} & \ldots & Y_{d-1} & Y_{d}
\end{array}\right)
$$

O Comentário 1.24 relaciona a regularidade de Castelnuovo-Mumford definida acima e a resolução minimal graduada de $M$ que está definida logo abaixo. Para mais detalhes, recomendamos $[\mathrm{BH}]$ e $[\mathrm{BS}]$.

Construamos agora uma sequência exata livre sobre $M$ que será chamada resolução minimal graduada de $M$. 
De fato, seja $M$ um módulo graduado finito sobre um anel ${ }^{*}$ local $(R, \mathfrak{m})$. Seja $g_{1}, \ldots, g_{n}$ um conjunto minimal de geradores homogêneos de $M$. Considere a seguinte soma direta $F_{0}=$ $\bigoplus_{i=1}^{n} R\left(-\operatorname{deg} g_{i}\right)$ que é gerada por elementos $e_{i}$ tais que deg $e_{i}=\operatorname{deg} g_{i}$. O $R_{0}$-módulo $F_{0}$ é livre de posto $n$. Além disso, podemos construir um morfismo sobrejetivo $\varphi_{0}$ de módulos graduados que faz corresponder $e_{i}$ sobre $g_{i}$. Veja que Ker $\varphi_{0}$ é um submódulo graduado de $F_{0}$. Afirmamos que Ker $\varphi_{0} \subseteq \mathfrak{m} F_{0}$. De fato, se ocorresse o contrário, então existe um elemento homogêneo $u \in \operatorname{Ker} \varphi_{0}$ tal que $u \notin \mathfrak{m} F_{0}$. Dessa forma, um dos coeficientes $a_{i}$ na decomposição $u=\sum_{i=1}^{n} a_{i} e_{i}$, que denotaremos por $a_{j}$, não pertence a m. Desde que todos os $a_{i}$ são homogêneos, temos que em particular $a_{j}$ é uma unidade (ver [BH, Lemma 1.5.7]). Daí, aplicando $\varphi_{0}$ a $u$, segue que o sistema de geradores dado não é minimal, o que é uma contradição. Se localizarmos a aplicação $\varphi_{0}$ em $\mathfrak{m}$, obtemos que $n=\mu\left(M_{\mathfrak{m}}\right)$. Donde segue que todo conjunto minimal de geradores homogêneos tem o mesmo número de elementos. Portanto, dando continuidade ao processo acima, obtemos o que é chamada de resolução minimal graduada livre de $M$. É fácil de mostrar que uma tal resolução é única a menos de isomorfismos homogêneos.

Comentário 1.24. [BS, Exercício 15.3.7] Seja $R:=k\left[X_{1}, \ldots, X_{n}\right]$ o anel de polinômios sobre um corpo $k$ em $n$ indeterminadas (que é graduado de forma natural). Note que $R e^{*}$ local com um único ideal ${ }^{*}$ maximal $\mathfrak{m}:=\left(X_{1}, \ldots, X_{n}\right)$. Seja $M$ um $R$-módulo graduado não nulo finito tal que $\operatorname{proj} \operatorname{dim} M=p$. Seja

$$
0 \rightarrow F_{p} \stackrel{f_{p}}{\rightarrow} F_{p-1} \rightarrow \ldots \rightarrow F_{1} \stackrel{f_{1}}{\rightarrow} F_{0} \stackrel{f_{0}}{\rightarrow} M \rightarrow 0
$$

uma resolução minimal graduada livre de $M$, tal que $F_{j}=\oplus_{i=1}^{b_{j}} R\left(a_{i}^{(j)}\right)$, onde $b_{0}, \ldots, b_{p} \in \mathbb{N} e$ para cada $j=0, \ldots, p, a_{1}^{(j)} \geq \ldots \geq a_{b_{j}}^{(j)}\left(i=1, \ldots, b_{j}\right)$. Então

(1) $\operatorname{reg}\left(F_{0}\right)=-a_{b_{0}}^{(0)}$;

(2) $-a_{b_{0}}^{(0)} \leq \operatorname{reg}(M)$;

(3) Se $p>0$ e $L:=\operatorname{Ker} f_{0}$, então $\operatorname{reg}(L) \leq \operatorname{reg}(M)+1$;

(4) $\operatorname{reg}(M)=\max \left\{-a_{b_{j}}^{(j)}-j: j=0, \ldots, p\right\}$.

No próximo exemplo vemos um caso particular em que os números $-a_{i}^{(j)}$ acima pertencem a um intervalo de comprimento igual a regularidade reg $M$. 
Exemplo 1.25. Consideramos a situação acima no caso em que $R=k\left[x_{1}, \ldots, x_{n}\right]$ é o anel dos polinômios sobre um corpo $k$, I um ideal graduado próprio de $R$ e $M=R / I$. Fazendo uso do fato que $\operatorname{Ker} f_{j} \subseteq \mathfrak{m} F_{j}$ para $j=0, \ldots, p$, podemos concluir que $b_{0}=1, a_{1}^{(0)}=0$ e que $-a_{1}^{(j+1)} \geq$ $-a_{1}^{(j)}+1$ para $j=0, \ldots, p-1$. Temos então que $-a_{1}^{(j)} \geq j$ para $j=0, \ldots, p$. Pelo Comentário 1.24(iv), temos que $-a_{b_{(j)}}^{j} \leq \operatorname{reg}(R / I)+j$ para $j=0, . ., p$. Portanto,

$$
-a_{i}^{(j)} \in\{r \in \mathbb{N} \cup\{0\} \mid j \leq r \leq \operatorname{reg}(R / I)+j\}
$$

para $j=0, \ldots, p$, onde $1 \leq i \leq b_{j}$.

Exemplo 1.26. Seja $R=k\left[x_{1}, \ldots, x_{n}\right]$ o anel dos polinômios sobre um corpo $k$ e $\mathfrak{m}$ seu ideal maximal graduado. O Comentário 1.24(4) e o Exemplo 1.19, dão que a regularidade de CastelnuovoMumford do corpo k é nula. Na verdade, para o anel $R=R_{0}\left[x_{1}, \ldots, x_{n}\right]$, onde $R_{0}$ é qualquer anel Noetheriano, temos $\operatorname{reg} R=0$ (ver [BS, Exercício 15.2.11]). 


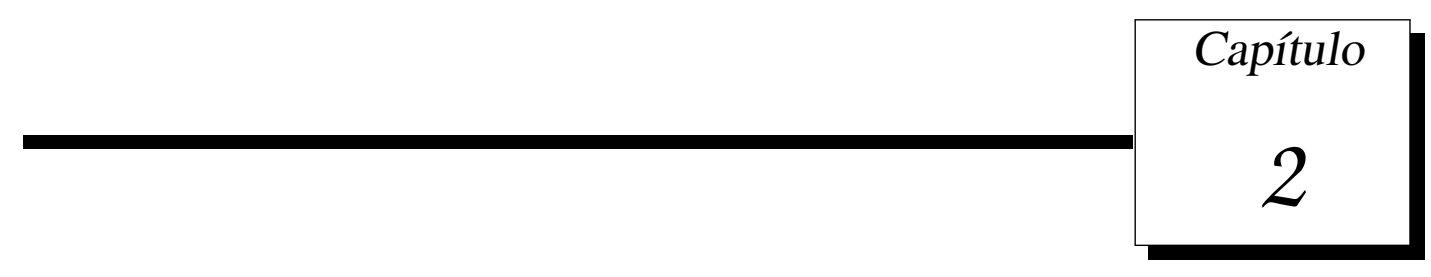

Regularidade da álgebra de Rees e do anel graduado associado para o caso de boas filtrações

Este capítulo trata das relações entre os invariantes cohomológicos do anel graduado associado e da álgebra de Rees. Mais especificamente, veremos que mesmo no caso de boas filtrações, os $a$-invariantes do anel graduado associado limitam superiormente os $a$-invariantes da álgebra de Rees, podendo também haver a igualdade sob certas condições.

Oishi prova em [O] que a regularidade de Castelnuovo-Mumford do anel graduado associado e da álgebra de Rees são iguais, isto é, $\operatorname{reg} G(I)=\operatorname{reg} R(I)$. Johnson e Ulrich, em [JU], redescobriram esta igualdade. Depois disso, no artigo [T], Trung mostra que existe uma relação próxima caracterizando o anulamento homogêneo da cohomologia da álgebra de Rees e do anel graduado associado, e então ele também mostra a igualdade acima. Neste capítulo, generalizamos resultados do artigo de Trung para o caso de filtrações, mostrando por exemplo que a igualdade acima, obtida inicialmente por Oishi, permanece válida no caso de boas filtrações em geral.

Seja $\mathfrak{F}$ uma filtração sobre um anel $R$. Consideramos tal anel como um anel graduado concentrado no grau zero. Agora considere as seguintes sequências exatas

$$
\begin{gathered}
0 \rightarrow R(\mathfrak{F})_{+} \rightarrow R(\mathfrak{F}) \rightarrow R \rightarrow 0 ; \\
0 \rightarrow R(\mathfrak{F})_{+}(1) \rightarrow R(\mathfrak{F}) \rightarrow G(\mathfrak{F}) \rightarrow 0 .
\end{gathered}
$$


Lema 2.1. Seja $\mathfrak{F}$ uma filtração sobre um anel $R$. Temos que

(1) se $i=0,1$, então $H_{R(\mathfrak{F})_{+}}^{i}(R(\mathfrak{F}))_{n}=0$ para $n \geq \max \left\{0, a_{i}(G(\mathfrak{F}))+1\right\}$,

(2) se $i \geq 2$, então $H_{R(\mathfrak{F})_{+}}^{i}(R(\mathfrak{F}))_{n}=0$ para $n \geq a_{i}(G(\mathfrak{F}))+1$.

Demonstração. Denotamos $H^{i}(\cdot)=H_{R(\mathfrak{F})_{+}}^{i}(\cdot)$. Como $H^{0}(R)=R$ e também $H^{i}(R)=0$ para $i \geq 1$, pela sequência exata (2.1) temos

$$
\begin{gathered}
H^{i}\left(R(\mathfrak{F})_{+}\right)_{n} \simeq H^{i}(R(\mathfrak{F}))_{n} \text { para } n=0, i \geq 2 \\
\text { e } H^{i}\left(R(\mathfrak{F})_{+}\right)_{n} \simeq H^{i}(R(\mathfrak{F}))_{n} \text { para } n \neq 0, i \geq 0 .
\end{gathered}
$$

Como $H_{G(\mathfrak{F})_{+}}^{i}(G(\mathfrak{F}))=H_{R(\mathfrak{F})_{+}}^{i}(G(\mathfrak{F}))$, a sequência exata (2.2) induz a sequência exata longa

$$
\cdots \rightarrow H^{i}\left(R(\mathfrak{F})_{+}\right)_{n+1} \rightarrow H^{i}(R(\mathfrak{F}))_{n} \rightarrow H^{i}(G(\mathfrak{F}))_{n} \rightarrow H^{i+1}\left(R(\mathfrak{F})_{+}\right)_{n+1} \rightarrow \cdots
$$

Então temos aplicações sobrejetivas

$$
\begin{gathered}
H^{i}(R(\mathfrak{F}))_{n+1} \rightarrow H^{i}(R(\mathfrak{F}))_{n} \text { para } n \geq \max \left\{0, a_{i}(G(\mathfrak{F}))+1\right\}, \text { se } i=0,1 \\
\text { e } H^{i}(R(\mathfrak{F}))_{n+1} \rightarrow H^{i}(R(\mathfrak{F}))_{n} \text { para } n \geq a_{i}(G(\mathfrak{F}))+1, \text { se } i \geq 2
\end{gathered}
$$

Pela Proposição A.22 temos $H^{i}(R(\mathfrak{F}))_{n}=0$ para $n \gg 0$, e então concluímos que

$$
\begin{gathered}
H^{i}(R(\mathfrak{F}))_{n}=0 \text { para } n \geq \max \left\{0, a_{i}(G(\mathfrak{F}))+1\right\}, \text { se } i=0,1 \\
\text { e } H^{i}(R(\mathfrak{F}))_{n}=0 \text { para } n \geq a_{i}(G(\mathfrak{F}))+1, \text { se } i \geq 2 .
\end{gathered}
$$

Teorema 2.2. Seja $\mathfrak{F}$ uma filtração sobre um anel R. Então

(1) $a_{i}(R(\mathfrak{F})) \leq a_{i}(G(\mathfrak{F}))$, para $i \neq 1$;

(2) $a_{i}(R(\mathfrak{F}))=a_{i}(G(\mathfrak{F}))$, se $a_{i}(G(\mathfrak{F})) \geq a_{i+1}(G(\mathfrak{F}))$ e $i \neq 1$;

(3) As afirmações (1) e (2) são verdadeiras para $i=1$, se $H_{G(\mathfrak{F})_{+}}^{1}(G(\mathfrak{F})) \neq 0$ ou $I_{1} \subseteq \sqrt{0}$;

(4) $a_{1}(R(\mathfrak{F}))=-1$, se $H_{G(\mathfrak{F})_{+}}^{1}(G(\mathfrak{F}))=0$ e $I_{1} \not \subset \sqrt{0}$; 
Demonstração. Denotamos $H^{i}(\cdot)=H_{R(\mathfrak{F})_{+}}^{i}(\cdot)$. Pelo Lema acima, $a_{i}(R(\mathfrak{F})) \leq a_{i}(G(\mathfrak{F}))$ para $i \geq 2$. Para $i=0$, temos dois casos. Se $H^{0}(G(\mathfrak{F}))=0$ então $a_{0}(G(\mathfrak{F}))=-\infty$. Assim, usando o Lema 2.1, temos $H^{0}(R(\mathfrak{F}))_{n}=0$ para $n \geq 0$. Mas desde que $H^{0}(R(\mathfrak{F}))$ é um ideal de $R(\mathfrak{F})$, temos que $H^{0}(R(\mathfrak{F}))=0$. Segue então que $a_{0}(R(\mathfrak{F}))=a_{0}(G(\mathfrak{F}))$. Se $H^{0}(G(\mathfrak{F})) \neq 0$, desde que $H^{0}(G(\mathfrak{F})) \subseteq G(\mathfrak{F})$ é um ideal, temos $a_{0}(G(\mathfrak{F})) \geq 0$. Assim, pelo Lema 2.1,

$$
H^{0}(R(\mathfrak{F}))_{n}=0 \text { para } n \geq a_{0}(G(\mathfrak{F}))+1 .
$$

Isso implica que $a_{0}(R(\mathfrak{F})) \leq a_{0}(G(\mathfrak{F}))$. Temos provado (1).

Agora mostremos (2). Usando (1), é suficiente mostrar que $a_{i}(R(\mathfrak{F})) \geq a_{i}(G(\mathfrak{F}))$. Dessa forma podemos supor que $a_{i}(G(\mathfrak{F})) \neq-\infty$. Se $i=0$, temos $H^{0}(G(\mathfrak{F})) \neq 0$, e então $a_{0}(G(\mathfrak{F})) \geq 0$. Devido a (2.3), temos a sequência exata longa

$$
\cdots \rightarrow H^{0}(R(\mathfrak{F}))_{a_{0}(G(\mathfrak{F}))} \rightarrow H^{0}(G(\mathfrak{F}))_{a_{0}(G(\mathfrak{F}))} \rightarrow H^{1}(R(\mathfrak{F}))_{a_{0}(G(\mathfrak{F}))+1} \rightarrow \cdots
$$

Pelo Lema 2.1, temos que $a_{1}(R(\mathfrak{F})) \leq-1$ ou $a_{1}(R(\mathfrak{F})) \leq a_{1}(G(\mathfrak{F}))$. No primeiro caso, desde que $a_{0}(G(\mathfrak{F})) \geq 0>-1 \geq a_{1}(R(\mathfrak{F}))$, obtemos $H^{1}(R(\mathfrak{F}))_{a_{0}(G(\mathfrak{F}))+1}=0$. Como $H^{0}(G(\mathfrak{F}))_{a_{0}(G(\mathfrak{F}))} \neq$ 0 , segue que $H^{0}(R(\mathfrak{F}))_{a_{0}(G(\mathfrak{F}))} \neq 0$, ou seja, $a_{0}(R(\mathfrak{F})) \geq a_{0}(G(\mathfrak{F}))$. Para o segundo caso, fazendo uso da hipótese $a_{0}(G(\mathfrak{F})) \geq a_{1}(G(\mathfrak{F}))$, temos $a_{1}(R(\mathfrak{F})) \leq a_{0}(G(\mathfrak{F}))$, de modo então que $H^{1}(R(\mathfrak{F}))_{a_{0}(G(\mathfrak{F}))+1}=0$. Segue similarmente ao primeiro caso que $a_{0}(R(\mathfrak{F})) \geq a_{0}(G(\mathfrak{F}))$. Agora se $i \geq 1$, por (1), têm-se $a_{i+1}(G(\mathfrak{F})) \geq a_{i+1}(R(\mathfrak{F}))$. Pela hipótese, obtemos $a_{i+1}(R(\mathfrak{F})) \leq$ $a_{i}(G(\mathfrak{F}))$. Então

$$
H^{i+1}\left(R(\mathfrak{F})_{+}\right)_{a_{i}(G(\mathfrak{F}))+1} \simeq H^{i+1}(R(\mathfrak{F}))_{a_{i}(G(\mathfrak{F}))+1}=0
$$

Dessa forma, a sequência exata (2.2) dá uma aplicação sobrejetiva

$$
H^{i}(R(\mathfrak{F}))_{a_{i}(G(\mathfrak{F}))} \rightarrow H^{i}(G(\mathfrak{F}))_{a_{i}(G(\mathfrak{F}))}
$$

Desde que $H^{i}(G(\mathfrak{F}))_{a_{i}(G(\mathfrak{F}))} \neq 0$, obtemos $H^{i}(R(\mathfrak{F}))_{a_{i}(G(\mathfrak{F}))} \neq 0$. Ou seja, $a_{i}(R(\mathfrak{F})) \geq a_{i}(G(\mathfrak{F}))$. Agora provemos (3). Se $H_{G(\mathfrak{F})_{+}}^{1}(G(\mathfrak{F})) \neq 0$, por [T, Corolário 2.3(iii)], temos $a_{1}(G(\mathfrak{F}))+$ $1 \geq 0$. Usando o Lema 2.1, conseguimos que $H^{1}(R(\mathfrak{F}))_{n}=0$ para $n \geq a_{1}(G(\mathfrak{F}))+1$. Segue então que $a_{1}(R(\mathfrak{F})) \leq a_{1}(G(\mathfrak{F}))$. Se $I_{1} \subseteq \sqrt{0}$, temos $R(\mathfrak{F})_{+} \subseteq \sqrt{0}$. Desde que $\operatorname{ara}\left(R(\mathfrak{F})_{+}\right)=$ 
$\operatorname{ara}\left(\sqrt{R(\mathfrak{F})_{+}}\right)=\operatorname{ara}(\sqrt{0})=0$, por [BS, Corolário 3.3.3], temos

$$
H^{i}(R(\mathfrak{F}))=0 \text { e } H^{i}(G(\mathfrak{F}))=0 \text {, para } i>0 .
$$

Em particular, $a_{1}(R(\mathfrak{F}))=a_{1}(G(\mathfrak{F}))$.

Agora, provemos (4). Assuma que $H^{1}(G(\mathfrak{F}))=0$ e $I_{1} \not \subset \sqrt{0}$. Então $a_{1}(G(\mathfrak{F}))=-\infty$. Pelo Lema 2.1, $a_{1}(R(\mathfrak{F})) \leq-1$. Suponha que $a_{1}(R(\mathfrak{F}))<-1$. Então $H^{1}(R(\mathfrak{F}))_{-1}=0$. É claro que $H^{0}(G(\mathfrak{F}))_{-1}=0$, uma vez que $H^{0}(G(\mathfrak{F})) \subseteq G(\mathfrak{F})$ é um ideal. A sequência exata (2.2), dá que $H^{1}\left(R(\mathfrak{F})_{+}\right)_{0}=0$. Pela sequência exata $(2.1)$, obtemos uma sequência exata

$$
H^{0}\left(R(\mathfrak{F})_{+}\right)_{0} \rightarrow H^{0}(R(\mathfrak{F}))_{0} \rightarrow H^{0}(R) \rightarrow 0
$$

Mas $H^{0}\left(R(\mathfrak{F})_{+}\right) \subseteq R(\mathfrak{F})_{+}$e $\left(R(\mathfrak{F})_{+}\right)_{0}=0, \operatorname{logo} H^{0}\left(R(\mathfrak{F})_{+}\right)_{0}=0$. É fácil mostrar a igualdade $H^{0}(R(\mathfrak{F}))_{0}=H_{I_{1}}^{0}(R)$. Também sabemos que $H^{0}(R)=R$. A sequência exata acima dá que $H_{I_{1}}^{0}(R)=R$, e isto implica que $I_{1}^{n}=0$ para algum $n \geq 1$, o que é uma contradição. Portanto $a_{1}(R(\mathfrak{F}))=-1$.

Corolário 2.3. Defina $s:=\max \left\{i: H_{G(\mathfrak{F})_{+}}^{i}(G(\mathfrak{F})) \neq 0\right\}$. Então

(1) $a_{s}(R(\mathfrak{F}))=a_{s}(G(\mathfrak{F}))$;

(2) $s=\max \left\{i: H_{R(\mathfrak{F})_{+}}^{i}(R(\mathfrak{F})) \neq 0\right\}$, se $I_{1} \subseteq \sqrt{0}$ ou $s \geq 1$.

Demonstração. Para $i \geq s$, temos $a_{i+1}(G(\mathfrak{F}))=-\infty$. Neste caso, sempre temos $a_{i}(G(\mathfrak{F})) \geq$ $a_{i+1}(G(\mathfrak{F}))$.

Se $i \neq 1$, pelo Teorema 2.2, temos $a_{i}(R(\mathfrak{F}))=a_{i}(G(\mathfrak{F}))$. Suponha então $s>1$. Assim $a_{s}(R(\mathfrak{F}))=a_{s}(G(\mathfrak{F}))$. Se $H_{R(\mathfrak{F})_{+}}^{s}(R(\mathfrak{F}))=0$, têm-se $a_{s}(R(\mathfrak{F}))=-\infty$, o que gera uma contradição sobre a hipótese de $s$. Logo

$$
H_{R(\mathfrak{F})_{+}}^{s}(R(\mathfrak{F})) \neq 0
$$

Se existe $j>s$ tal que $H_{R(\mathfrak{F})_{+}}^{j}(R(\mathfrak{F})) \neq 0$, pelo Teorema 2.2(2), $a_{j}(G(\mathfrak{F}))=a_{j}(R(\mathfrak{F}))$, o que é uma contradição, já que $H_{G(\mathfrak{F})_{+}}^{j}(G(\mathfrak{F}))=0$. Portanto se $s>1$, o resultado segue. Se $s=1$, $H_{G(\mathfrak{F})_{+}}^{1}(G(\mathfrak{F})) \neq 0$. Pelo Teorema 2.2(3), $a_{1}(R(\mathfrak{F}))=a_{1}(G(\mathfrak{F}))$. Se $H_{R(\mathfrak{F})_{+}}^{1}(R(\mathfrak{F}))=0$ então 
$a_{1}(R(\mathfrak{F}))=-\infty$, o que leva a uma contradição. $\operatorname{Logo} H_{R(\mathfrak{F})_{+}}^{1}(R(\mathfrak{F})) \neq 0$. Se existe $j>1$ tal que $H_{R(\mathfrak{F})_{+}}^{j}(R(\mathfrak{F})) \neq 0$, o Teorema 2.2(2) nos leva a uma contradição.

Agora iremos provar o último caso. Suponha que $s=0$ e $I_{1} \subseteq \sqrt{0}$. Pelo Teorema 2.2(2), $a_{0}(R(\mathfrak{F}))=a_{0}(G(\mathfrak{F}))$. Se $H_{R(\mathfrak{F})_{+}}^{0}(R(\mathfrak{F}))=0$ então $a_{0}(R(\mathfrak{F}))=-\infty$, o que não pode ocorrer.

Corolário 2.4. Seja $R$ um anel Noetheriano local e $\mathfrak{F}$ uma boa filtração. Então

$$
\operatorname{reg} R(\mathfrak{F})=\operatorname{reg} G(\mathfrak{F})
$$

Demonstração. Pelo Teorema 2.2(1), $a_{i}(R(\mathfrak{F}))+i \leq a_{i}(G(\mathfrak{F}))+i$, para $i \neq 1$. Lembre que por [HZ, Proposição 3.2], reg $G(\mathfrak{F}) \geq 0$. Pelo Teorema 2.2(3)(4),

$$
a_{1}(R(\mathfrak{F}))+1 \leq a_{1}(G(\mathfrak{F}))+1 \text { ou } a_{1}(R(\mathfrak{F}))+1=0 \leq \operatorname{reg} G(\mathfrak{F})
$$

Em ambos os casos, temos $\operatorname{reg} R(\mathfrak{F}) \leq \operatorname{reg} G(\mathfrak{F})$.

Agora vamos provar a outra desigualdade. Seja $i$ maximal tal que

$$
a_{i}(G(\mathfrak{F}))+i=\operatorname{reg} G(\mathfrak{F})
$$

Isso implica que $a_{i}(G(\mathfrak{F}))+i \geq a_{i+1}(G(\mathfrak{F}))+i+1$, ou seja $a_{i}(G(\mathfrak{F}))>a_{i+1}(G(\mathfrak{F}))$. Se tivermos $H_{G(\mathfrak{F})_{+}}^{i}(G(\mathfrak{F}))=0$, então $a_{i}(G(\mathfrak{F}))=-\infty$, o que é uma contradição. Logo

$$
H_{G(\mathfrak{F})_{+}}^{i}(G(\mathfrak{F})) \neq 0
$$

Se $i \neq 1$, temos $a_{i}(R(\mathfrak{F}))=a_{i}(G(\mathfrak{F}))$. Se $i=1$, pelo Teorema 2.2(3), $a_{1}(R(\mathfrak{F}))=a_{1}(G(\mathfrak{F}))$, já que $H_{G(\mathfrak{F})_{+}}^{1}(G(\mathfrak{F})) \neq 0$. Logo $a_{i}(R(\mathfrak{F}))=a_{i}(G(\mathfrak{F}))$, para $i \geq 0$. Donde concluímos que

$$
\operatorname{reg} G(\mathfrak{F})=a_{i}(R(\mathfrak{F}))+i \leq \operatorname{reg} R(\mathfrak{F})
$$





\section{- \\ Regularidade da fibra especial para boas filtrações}

A regularidade de Castelnuovo-Mumford é um importante invariante na geometria algébrica, pois faz conexões entre a teoria de cohomologia local e os syzygies de módulos graduados finitos sobre anéis de polinômios (sobre um corpo).

No capítulo passado encontramos relações entre os $a$-invariantes cohomológicos da regularidade do anel graduado associado $G(\mathfrak{F})$ e da álgebra de Rees $R(\mathfrak{F})$. É natural perguntar por exemplo, sob que condições teremos também a igualdade entre a regularidade da fibra especial $F(\mathfrak{F})$ e da álgebra de Rees $R(\mathfrak{F})$. No artigo [CZ3], Cortadellas e Zarzuela provam, para um ideal $I$ tal que $h(I)=s(I)$ (onde $h(I)$ denota a altura do ideal $I$ e $s(I)$ denota a extensão analítica do ideal $I$ ), que se a profundidade do anel graduado associado $G(I)$ e da fibra especial $F(I)$ são limitadas inferiormente por $s-1$, então as regularidades dessas álgebras são iguais. No artigo [JN], Jayanthan e Nanduri generalizam esse resultado, mostrando que a igualdade é verdadeira para ideais em geral. Neste capítulo, mostramos que este resultado permanece válido no caso de boas filtrações e além disso, temos acrescentado novos resultados a este teoria.

Neste capítulo, $R$ será um anel local Noetheriano de dimensão $d>0$ com corpo residual infinito $R / \mathfrak{m}$ e $\mathfrak{F}$ uma boa filtração. Considere as filtrações

$$
\begin{aligned}
& \mathscr{F}: R \supset \mathfrak{m} \supset \mathfrak{m} I_{1} \supset \mathfrak{m} I_{2} \supset \ldots \\
& \mathfrak{F}: R \supset I_{1} \supset I_{2} \supset I_{3} \supset \ldots .
\end{aligned}
$$

Durante este capítulo, assumimos que $I_{n+1} \subseteq \mathfrak{m} I_{n}$, para todo $n$. Esta suposição é bastante natural. 
Por exemplo, se depth $F(\mathfrak{F})>0$, então essa inclusão é válida devido a [CZ, Lema 3.1].

Desde que existe $k$ inteiro positivo tal que $\mathfrak{m} I_{k+n-1}=\mathfrak{m} I_{k-1} I_{n}$, para $n \geq k$, não é difícil mostrar que $R(\mathscr{F})$ é um $R(\mathfrak{F})$-módulo finito. Assim, temos duas sequências exatas curtas

$$
\begin{gathered}
0 \rightarrow R(\mathfrak{F}) \rightarrow R(\mathscr{F}) \rightarrow \mathfrak{m} G(\mathfrak{F})(-1) \rightarrow 0 \\
0 \rightarrow \mathfrak{m} G(\mathfrak{F}) \rightarrow G(\mathfrak{F}) \rightarrow F(\mathfrak{F}) \rightarrow 0
\end{gathered}
$$

de $R(\mathfrak{F})$-módulos finitos. Note que $\mathfrak{m} G(\mathfrak{F})$ é um $R(\mathfrak{F})$-submódulo finito já que $G(\mathfrak{F})$ é um $R(\mathfrak{F})$ módulo Noetheriano. Neste capítulo, sempre consideramos $s:=s(\mathfrak{F})=\operatorname{dim} F(\mathfrak{F})>0$.

Lema 3.1. Sejam $\underline{x}=x_{1}, \ldots, x_{s}$ geradores de uma redução minimal de $\mathfrak{F}$. Denote $\underline{x}^{k}=\left(x_{1}^{k}, \ldots, x_{s}^{k}\right)$. Temos que $a_{s}(R(\mathscr{F}))-1 \leq a_{s}(R(\mathfrak{F}))$.

Demonstração. É fácil mostrar que $\sqrt{R(\mathfrak{F})_{+}}=\sqrt{\left(x_{1} t, \ldots, x_{s} t\right)}$. Pela Proposição A.14 e [HIO, Corolário 35.21], obtemos

$$
\left[H_{R(\mathfrak{F})_{+}}^{s}(R(\mathfrak{F}))\right]_{n} \cong \lim _{\vec{k}} \frac{I_{s k+n}}{\left(\underline{x^{k}}\right) I_{(s-1) k+n}}
$$

$\mathrm{e}$

$$
\left[H_{R(\mathfrak{F})_{+}}^{s}(R(\mathscr{F}))\right]_{n} \cong \lim _{\vec{k}} \frac{\mathfrak{m} I_{s k+n-1}}{\left(\underline{x^{k}}\right) \mathfrak{m} I_{(s-1) k+n-1}}
$$

Agora, fazendo uso da propriedade de limite para sequências exatas, podemos obter que $a_{s}(R(\mathscr{F}))-1 \leq a_{s}(R(\mathfrak{F}))$.

Em [HZ], Hoa e Zarzuela mostram que se $\mathfrak{F}$ é uma boa filtração equimúltipla de um anel de Cohen-Macaulay e grade $G(\mathfrak{F})_{+} \geq s(\mathfrak{F})-1$, então a regularidade $\operatorname{reg} F(\mathfrak{F})$ da fibra especial é igual ao número de redução $r(\mathfrak{F})$. Abaixo, vemos o mesmo resultado mas sobre outras hipóteses. Consideramos o caso que a extensão analítica da filtração $\mathfrak{F}$ é um.

Lema 3.2. Sejam $(R, \mathfrak{m})$ um anel local Noetheriano e $\mathfrak{F}$ uma boa filtração tal que $s(\mathfrak{F})=1$. Se $J$ é uma redução minimal de $\mathfrak{F}$ e grade $I_{1} \geq 1$, então

$$
\operatorname{reg} F(\mathfrak{F})=r_{J}(\mathfrak{F})
$$


Demonstração. Pelo Teorema A.5, temos que $s(\mathfrak{F})=\mu(J)$ (onde $\mu(J)$ denota o número minimal de geradores do ideal $J$ ), e então por hipótese, $J=(a)$. Novamente, usando o Teorema A.5, obtemos que $a$ é analiticamente independente sobre $\mathfrak{F}$. Pelo comentário A.4, $F(J)$ é isomorfo a $k[x]$ (onde $k=R / \mathfrak{m}$ ), desde que $a$ também é analiticamente independente em $I_{1}$. Além disso, $J^{i} \cap I_{i} \mathfrak{m}=J^{i} \mathfrak{m}$ para todo $i$. Então o morfismo

$$
F(J) \hookrightarrow F(\mathfrak{F})
$$

é finito (desde que $R(\mathfrak{F})$ é um $R(J)$-módulo finito). Agora por [V, Exemplo 9.3.1] ou [GP, Teorema 2.6.1] (versão graduada),

$$
F(\mathfrak{F}) \simeq \bigoplus_{i=1}^{e} F(J)\left(-b_{i}\right) \bigoplus_{j=1}^{f}\left(F(J) / a^{c_{j}} F(J)\right)\left(-d_{j}\right)
$$

onde $c_{j} \geq 1$ para cada $j$. Assumamos que $b_{1} \leq \ldots \leq b_{e}$ e $d_{1} \leq \ldots \leq d_{f}$. Assim

$$
H_{F(\mathfrak{F})}(x)=\frac{x^{b_{1}}+\ldots+x^{b_{e}}+\left(1-x^{c_{1}}\right) x^{d_{1}}+\ldots+\left(1-x^{c_{f}}\right) x^{d_{f}}}{1-x}
$$

Observe que

$$
\begin{aligned}
& r_{J}(\mathfrak{F})=\max \left\{b_{e}, d_{f}\right\} \\
& \operatorname{reg} F(\mathfrak{F})=\max \left\{b_{e}, c_{j}+d_{j}-1\right\} .
\end{aligned}
$$

Por hipótese, $I_{1}$ contém um elemento regular. Note que desde que $J$ é uma redução de $I_{1}$, o elemento $a$ é também regular. Seja $r:=r_{J}(\mathfrak{F})$. Se $n \geq r$ então $I_{n}=a^{n-r} I_{r}$. Assim

$$
\frac{I_{n}}{\mathfrak{m} I_{n}}=\frac{a^{n-r} I_{r}}{a^{n-r} \mathfrak{m} I_{r}}
$$

e então temos uma aplicação

$$
\frac{I_{r}}{\mathfrak{m} I_{r}} \rightarrow \frac{I_{n}}{\mathfrak{m} I_{n}}
$$

definida pela multiplicação por $a^{n-r}$. É fácil de mostrar que esta aplicação é um isomorfismo. 
Logo, $\mu\left(I_{n}\right)=\mu\left(I_{r}\right)$ para $n \geq r$. Então

$$
H_{F(\mathfrak{F})}(x)=\sum_{n \geq 0} \mu\left(I_{n}\right) x^{n}=\frac{1+\left(\mu\left(I_{1}\right)-1\right) x+\ldots+\left(\mu\left(I_{r}\right)-\mu\left(I_{r-1}\right)\right) x^{r}}{1-x}
$$

Comparando ambas as expressões das séries de Hilbert, segue que $c_{j}+d_{j} \leq r$, e então $d_{f} \leq r-1$. Em particular, $r=r_{J}(\mathfrak{F})=b_{e}$. Portanto, por $(3.3), \operatorname{reg} F(\mathfrak{F})=r_{J}(\mathfrak{F})$.

No artigo [JN], Jayanthan e Nanduri mostram que se a extensão analítica é um, a regularidade da fibra especial $F(I)$ é limitada superiormente pela regularidade do anel graduado associado $G(I)$. O próximo teorema mostra que este resultado permanece verdadeiro para boas filtrações. Denotamos por $H^{i}(M)$, o $i$-ésimo módulo de cohomologia local $H_{R(\mathfrak{F})_{+}}^{i}(M)$ do módulo $M$ com respeito a $R(\mathfrak{F})_{+}$.

Antes de enunciar o Teorema 3.3, é importante dizer que podemos alterar o suporte $R(\mathfrak{F})_{+}$ do módulo de cohomologia por $G(\mathfrak{F})_{+}$, pois de fato, usando [HIO, Corolário 35.20], podemos concluir que $H_{R(\mathfrak{F})_{+}}^{i}(M)=H_{G(\mathfrak{F})_{+}}^{i}(M)$.

Teorema 3.3. Seja $(R, \mathfrak{m})$ um anel local Noetheriano e $\mathfrak{F}$ uma boa filtração tal que $s:=s(\mathfrak{F})=1$. Então $\operatorname{reg} F(\mathfrak{F}) \leq \operatorname{reg} G(\mathfrak{F})$. Além disso, se grade $I_{1}=1$, temos que $\operatorname{reg} F(\mathfrak{F})=\operatorname{reg} G(\mathfrak{F})=r(\mathfrak{F})$.

Demonstração. Desde que $s=1, \sqrt{R(\mathfrak{F})_{+}}=\sqrt{\left(x_{1} t\right)}$, onde $x_{1}$ gera uma redução minimal de $\mathfrak{F}$. Como $H_{R(\mathfrak{F})_{+}}^{i}(M)=0$ para $i>\operatorname{ara}\left(R(\mathfrak{F})_{+}\right)$e $\operatorname{ara}\left(R(\mathfrak{F})_{+}\right) \leq 1$, segue que $H^{i}(M)=0$ para $i>1$. Pela sequência (3.2), temos a seguinte sequência exata longa

$$
\begin{aligned}
0 & \rightarrow H^{0}(\mathfrak{m} G(\mathfrak{F})) \rightarrow H^{0}(G(\mathfrak{F})) \rightarrow H^{0}(F(\mathfrak{F})) \\
& \rightarrow H^{1}(\mathfrak{m} G(\mathfrak{F})) \rightarrow H^{1}(G(\mathfrak{F})) \rightarrow H^{1}(F(\mathfrak{F})) \rightarrow 0 .
\end{aligned}
$$

Assim $a_{0}(\mathfrak{m} G(\mathfrak{F})) \leq a_{0}(G(\mathfrak{F}))$ e $a_{1}(F(\mathfrak{F})) \leq a_{1}(G(\mathfrak{F}))$. Pela sequência exata (3.1), temos a sequência exata longa

$$
\begin{aligned}
0 & \rightarrow H^{0}(R(\mathfrak{F})) \rightarrow H^{0}(R(\mathscr{F})) \rightarrow H^{0}(\mathfrak{m} G(\mathfrak{F}))(-1) \\
& \rightarrow H^{1}(R(\mathfrak{F})) \rightarrow H^{1}(R(\mathscr{F})) \rightarrow H^{1}(\mathfrak{m} G(\mathfrak{F}))(-1) \rightarrow 0 .
\end{aligned}
$$


Dessa forma $a_{1}(\mathfrak{m} G(\mathfrak{F})(-1))=a_{1}(\mathfrak{m} G(\mathfrak{F}))+1 \leq a_{1}(R(\mathscr{F}))$ e pelo Lema 3.1, temos

$$
a_{1}(\mathfrak{m} G(\mathfrak{F})) \leq a_{1}(R(\mathfrak{F}))
$$

Desde que $s=1$, pelo comentário em [T, p. 2818], temos $H_{G(\mathfrak{F})_{+}}^{1}(G(\mathfrak{F})) \neq 0$. Assim, pelo Teorema 2.2, temos $a_{1}(R(\mathfrak{F})) \leq a_{1}(G(\mathfrak{F}))$, de modo que $a_{1}(\mathfrak{m} G(\mathfrak{F})) \leq a_{1}(G(\mathfrak{F}))$. Portanto

$$
\operatorname{reg} \mathfrak{m} G(\mathfrak{F}) \leq \operatorname{reg} G(\mathfrak{F})
$$

Usando a sequência exata (3.2) e o Proposição 1.21, obtemos

$$
\operatorname{reg} F(\mathfrak{F}) \leq \max \{\operatorname{reg} G(\mathfrak{F}), \operatorname{reg} \mathfrak{m} G(\mathfrak{F})-1\}=\operatorname{reg} G(\mathfrak{F})
$$

Agora, supomos que grade $I_{1}=1$. Pelo Lema 3.2, temos $\operatorname{reg} F(\mathfrak{F})=r_{J}(\mathfrak{F})$ para qualquer redução minimal $J$ de $\mathfrak{F}$. Por [HZ, Proposição 3.6], têm-se $\operatorname{reg} G(\mathfrak{F})=r_{J}(\mathfrak{F})$ e portanto $\operatorname{reg} F(\mathfrak{F})=\operatorname{reg} G(\mathfrak{F})=r(\mathfrak{F})$.

Corolário 3.4. Seja $(R, \mathfrak{m})$ um anel local Noetheriano e $\mathfrak{F}$ uma boa filtração equimúltipla tal que $s(\mathfrak{F}):=\operatorname{dim} F(\mathfrak{F})=1$. Se Ré Cohen-Macaulay, então $\operatorname{reg} F(\mathfrak{F})=\operatorname{reg} G(\mathfrak{F})=r(\mathfrak{F})$.

Demonstração. Desde que $R$ é Cohen-Macaulay e $\mathfrak{F}$ é equimúltipla, por [HZ, Lema 2.8] e [GP, Corolário 7.7.10], temos $s(\mathfrak{F})=s\left(I_{1}\right)=\operatorname{ht}\left(I_{1}\right)=$ grade $I_{1}$.

O teorema abaixo generaliza a primeira parte do Teorema 3.3 e como consequência, a conclusão adicional do Teorema 3.10 estará concluída.

Teorema 3.5. Seja $(R, \mathfrak{m})$ um anel Noetheriano local e $\mathfrak{F}$ uma boa filtração onde $s=s(\mathfrak{F})$. Se $\operatorname{depth} F(\mathfrak{F}) \geq s-1$ então $\operatorname{reg} F(\mathfrak{F}) \leq \operatorname{reg} G(\mathfrak{F})$.

Demonstração. Temos que $H^{i}(M)=0$ para $i>s$, onde $M$ é qualquer $R(\mathfrak{F})$-módulo. A sequência exata longa

$$
\cdots \rightarrow H^{s}(R(\mathfrak{F})) \rightarrow H^{s}(R(\mathscr{F})) \rightarrow H^{s}(\mathfrak{m} G(\mathfrak{F}))(-1) \rightarrow 0
$$


$\operatorname{diz}$ que $a_{s}(\mathfrak{m} G(\mathfrak{F})(-1))=a_{s}(\mathfrak{m} G(\mathfrak{F}))+1 \leq a_{s}(R(\mathscr{F}))$. Então pelo Lema 3.1 temos $a_{s}(\mathfrak{m} G(\mathfrak{F})) \leq$ $a_{s}(R(\mathfrak{F}))$. O caso $s=1$ já foi feito no Teorema 3.3. Suponhamos que $s \neq 1$. Pelo Teorema 2.2, temos $a_{s}(\mathfrak{m} G(\mathfrak{F})) \leq a_{s}(G(\mathfrak{F}))$. Temos também a seguinte sequência exata

$$
\begin{aligned}
& 0 \rightarrow H^{s-1}(\mathfrak{m} G(\mathfrak{F})) \rightarrow H^{s-1}(G(\mathfrak{F})) \rightarrow H^{s-1}(F(\mathfrak{F})) \rightarrow \\
& \rightarrow H^{s}(\mathfrak{m} G(\mathfrak{F})) \rightarrow H^{s}(G(\mathfrak{F})) \rightarrow H^{s}(F(\mathfrak{F})) \rightarrow 0 .
\end{aligned}
$$

Então $a_{s-1}(\mathfrak{m} G(\mathfrak{F})) \leq a_{s-1}(G(\mathfrak{F}))$. Podemos então concluir que $\operatorname{reg} \mathfrak{m} G(\mathfrak{F}) \leq \operatorname{reg} G(\mathfrak{F})$ e portanto

$$
\operatorname{reg} F(\mathfrak{F}) \leq \max \{\operatorname{reg} G(\mathfrak{F}), \operatorname{reg} \mathfrak{m} G(\mathfrak{F})-1\}=\operatorname{reg} G(\mathfrak{F})
$$

O Teorema 3.3 e o Teorema 3.5 sugerem a seguinte questão, a qual não fomos capazes de responder.

Questão 3.6. Seja $(R, \mathfrak{m})$ um anel Noetheriano local e $\mathfrak{F}$ uma boa filtração onde $s=s(\mathfrak{F})$. Perguntamos então: Se grade $I_{1}=s e \operatorname{depth} F(\mathfrak{F}) \geq s-1$ então $\operatorname{reg} F(\mathfrak{F})=\operatorname{reg} G(\mathfrak{F})$ ?

Proposição 3.7. Seja $(R, \mathfrak{m})$ um anel local Noetheriano de dimensão $d>0$ e $\mathfrak{F}$ uma boa filtração. Então existe uma redução minimal $J=\left(x_{1}, \ldots, x_{s}\right)$ de $\mathfrak{F}$, tal que $x_{1}^{o}, \ldots, x_{s}^{o} \in I_{1} / \mathfrak{m} I_{1}$ é uma sequência superficial para $F(\mathfrak{F})$ e $x_{1}^{*}, \ldots, x_{s}^{*} \in I_{1} / I_{2}$ é uma sequência superficial para $G(\mathfrak{F})$.

Demonstração. Recorrendo à indução, mostremos somente o caso em que $s=1$. Primeiramente, consideramos os ideais primos associados do anel $G(\mathfrak{F})$ e da fibra $F(\mathfrak{F})$, respectivamente, como sendo

$$
\operatorname{Ass}(G(\mathfrak{F}))=\left\{\mathfrak{p}_{1}, \ldots, \mathfrak{p}_{r}, \mathfrak{p}_{r+1}, \ldots, \mathfrak{p}_{s}\right\} \text { e } \operatorname{Ass}(F(\mathfrak{F}))=\left\{\mathfrak{q}_{1}, \ldots, \mathfrak{q}_{l}, \mathfrak{q}_{l+1}, \ldots, \mathfrak{q}_{m}\right\}
$$

e tais que para $n \gg 0, I_{n} / I_{n+1} \subseteq \mathfrak{p}_{i}$ para $r+1 \leq i \leq s$ e $I_{n} / \mathfrak{m} I_{n} \subseteq \mathfrak{q}_{j}$ para $l+1 \leq j \leq m$. Sabemos que o anel graduado associado $G(\mathfrak{F})$ e a fibra especial $F(\mathfrak{F})$ são ambos quocientes da álgebra de Rees estendida, que denotaremos por $\mathscr{R}=R(\mathfrak{F})\left(t^{-1}\right)$. Dessa forma, tomemos $\mathscr{P}=\left\{\mathfrak{p}_{1}^{\prime}, \ldots, \mathfrak{p}_{s}^{\prime}, \mathfrak{q}_{1}^{\prime}, \ldots, \mathfrak{q}_{m}^{\prime}\right\}$ como sendo as pré-imagens dos ideais primos $\mathfrak{p}_{1}, \ldots, \mathfrak{p}_{r}, \mathfrak{p}_{r+1}, \ldots, \mathfrak{p}_{s}$ 
em $G(\mathfrak{F})$ e $\mathfrak{q}_{1}, \ldots, \mathfrak{q}_{l}, \mathfrak{q}_{l+1}, \ldots, \mathfrak{q}_{m}$ em $F(\mathfrak{F})$, respectivamente. Desde que $R / \mathfrak{m}$ é infinito, um argumento de álgebra linear, nos dá que $\mathscr{R}_{1} \nsubseteq \mathfrak{m} I_{1} t \cup \mathfrak{p}_{1} \cup \cdots \cup \mathfrak{p}_{r} \cup \mathfrak{q}_{1} \cup \cdots \cup \mathfrak{q}_{l}$. Assim, seja $x \in \mathscr{R}_{1}$ tal que $x \notin \mathfrak{m} I_{1} t \cup \mathfrak{p}_{1} \cup \cdots \cup \mathfrak{p}_{r} \cup \mathfrak{q}_{1} \cup \cdots \cup \mathfrak{q}_{l}$. Agora mostremos que $0 \neq x^{o} \in F(\mathfrak{F})$ é superficial para $F(\mathfrak{F})$.

Precisamos mostrar que $\left(0: x^{o}\right) \cap F(\mathfrak{F})_{n}=0$ para $n \gg 0$. Seja $y \in\left(0: x^{o}\right)$. Agora consideremos a decomposição primária $(0)=N_{1} \cap \cdots \cap N_{m}$ de $(0)$ em $F(\mathfrak{F})$, tal que $N_{j}$ seja $\mathfrak{q}_{j}$-primário para $j=1, \ldots, m$. Donde segue que $y x^{o} \in N_{j}$ para $j=1, \ldots, l$ e $x^{o} \notin \mathfrak{q}_{j} \supseteq N_{j}$ para $j=1, \ldots, l$. Logo $y \in N_{j}$ para $j=1, . ., l$. Temos mostrado que $\left(0: x^{o}\right) \subseteq N_{1} \cap \cdots N_{l}$. Desde que $I_{n} / \mathfrak{m} I_{n} \subseteq \mathfrak{q}_{j}$ para $n \gg 0$, onde $l+1 \leq j \leq m$, concluímos que

$$
\left(0: x^{o}\right) \cap F(\mathfrak{F})_{n} \subseteq N_{1} \cap \cdots N_{l} \cap N_{l+1} \cap \cdots \cap N_{m}=(0),
$$

para $n$ suficientemente grande. Similarmente, mostra-se que $x^{*} \in G(\mathfrak{F})$ é superficial para $G(\mathfrak{F})$.

Lema 3.8. Seja $(R, \mathfrak{m})$ um anel local e $\mathfrak{F}$ um boa filtração tal que a $\in I_{1}$. Se a*é um elemento regular de $G(\mathfrak{F})$, então

$$
\frac{F(\mathfrak{F})}{a^{o} F(\mathfrak{F})} \cong F(\mathfrak{F} /(a))
$$

Demonstração. Desde que $a^{*}$ é um elemento regular de $G(\mathfrak{F})$, é fácil mostrar que $a R \cap I_{n}=a I_{n-1}$ para qualquer $n \geq 0$. Note que

$$
F(\mathfrak{F} /(a))=\bigoplus_{n \geq 0} \frac{\frac{I_{n}+a R}{a R}}{\frac{\mathfrak{m}}{a R}\left(\frac{I_{n}+a R}{a R}\right)}=\bigoplus_{n \geq 0} \frac{\frac{I_{n}+a R}{a R}}{\frac{\mathfrak{m} I_{n}+a R}{a R}} \simeq \bigoplus_{n \geq 0} \frac{I_{n}+a R}{\mathfrak{m} I_{n}+a R}
$$

A aplicação natural

$$
I_{n} \longrightarrow \frac{I_{n}+a R}{\mathfrak{m} I_{n}+a R}
$$

induz um isomorfismo

$$
\frac{I_{n}}{\mathfrak{m} I_{n}+\left(I_{n} \cap a R\right)} \simeq \frac{I_{n}+a R}{\mathfrak{m} I_{n}+a R}
$$

Note que

$$
\left(\frac{F(\mathfrak{F})}{a^{o} F(\mathfrak{F})}\right)_{n}=\frac{F(\mathfrak{F})_{n}}{\left(a^{o} F(\mathfrak{F})\right)_{n}}=\frac{\frac{I_{n}}{\mathfrak{m} I_{n}}}{\frac{a I_{n-1}+\mathfrak{m} I_{n}}{\mathfrak{m} I_{n}}} \simeq \frac{I_{n}}{a I_{n-1}+\mathfrak{m} I_{n}}=\frac{I_{n}}{\left(a R \cap I_{n}\right)+\mathfrak{m} I_{n}}
$$


o que conclui o resultado.

Antes dar início ao próximo resultado, relembremos a definição de multiplicidade de um módulo graduado finito.

Seja $M$ um $R$-módulo graduado finito, onde $R$ é um anel Noetheriano posivitamente graduado gerado por elementos de grau um e tal que $R_{0}$ seja um anel local Artiniano. Definimos a função de Hilbert do módulo $M$ como sendo $H(M, n)=\ell\left(M_{n}\right)$, para todo $n \in \mathbb{Z}$. É bem conhecido que para $n \gg 0$, a função $H(M, n)$ é um polinômio em $n$ de grau $d-1$. Esse polinômio, o qual denotamos por $P(M, n)$, pode ser escrito na forma

$$
P(M, n)=\sum_{n \geq 0}^{d-1}(-1)^{d-1-i} e_{d-1-i}\left(\begin{array}{c}
n+i \\
i
\end{array}\right)
$$

onde $e_{i} \in \mathbb{Z}$ para todo $i$. O inteiro $e_{0}:=e_{0}(M)$ é chamado a multiplicidade (de Hilbert) do módulo $M$.

O próximo resultado será de grande utilidade no próximo teorema. Perceba que o Lema abaixo, por indução, é na verdade válido para sequências superficiais da fibra especial $F(\mathfrak{F})$.

Lema 3.9. Se $x^{o} \in F(\mathfrak{F})$ é superficial então $\operatorname{dim} F(\mathfrak{F}) / x^{o} F(\mathfrak{F})=\operatorname{dim} F(\mathfrak{F})-1$.

Demonstração. Primeiramente consideramos a seguinte sequência exata

$$
0 \rightarrow\left(\mathfrak{m} I_{n}: x\right) \cap I_{n-1} / \mathfrak{m} I_{n-1} \rightarrow I_{n-1} / \mathfrak{m} I_{n-1} \stackrel{x}{\rightarrow} I_{n} / \mathfrak{m} I_{n} \rightarrow I_{n} /\left(\mathfrak{m} I_{n}+x I_{n-1}\right) \rightarrow 0
$$

Por aditividade, $\ell\left(I_{n} / \mathfrak{m} I_{n}\right)-\ell\left(I_{n-1} / \mathfrak{m} I_{n-1}\right)=\ell\left(I_{n} /\left(\mathfrak{m} I_{n}+x I_{n-1}\right)\right)-\ell\left(\left(\mathfrak{m} I_{n}: x\right) \cap I_{n-1} / \mathfrak{m} I_{n-1}\right)$, para todo $n \geq 1$. Note que $\left(F(\mathfrak{F}) / x^{o} F(\mathfrak{F})\right)_{n}=I_{n} /\left(\mathfrak{m} I_{n}+x I_{n-1}\right)$. Desde que $x^{o} \in F(\mathfrak{F})$ é superficial, temos $\left(\mathfrak{m} I_{n}: x\right) \cap I_{n-1}=\mathfrak{m} I_{n-1}$ para $n \gg 0$. Dessa forma, para $n$ suficientemente grande, temos a seguinte igualdade de polinômios de Hilbert:

$$
P\left(F(\mathfrak{F}) / x^{o} F(\mathfrak{F}), n\right)=P(F(\mathfrak{F}), n)-P(F(\mathfrak{F}), n-1)
$$

Concluímos então que $\operatorname{dim} F(\mathfrak{F}) / x^{o} F(\mathfrak{F})=\operatorname{dim} F(\mathfrak{F})-1$.

Agora é natural perguntar se a regularidade da fibra especial pode ser maior que a regularidade do anel graduado associado e sobre quais condições a igualdade ocorre. No artigo [CZ3], 
Cortadellas e Zarzuela provam para o caso de filtrações adicas, que se a profundidade da fibra especial $F(I)$, depth $F(I)$, e do anel graduado associado $G(I)$, depth $G(I)$, é no mínimo $s(I)-1$, suas regularidades são iguais. Em [JN], Jayanthan e Nanduri generalizam este resultado no caso $I$-adico. Verifiquemos agora que ele também permanece válido para o caso de boas filtrações.

Teorema 3.10. Seja $(R, \mathfrak{m})$ um anel local Noetheriano de Cohen-Macaulay e $\mathfrak{F}$ uma boa filtração tal que $s:=s(\mathfrak{F})$. Suponha que grade $I_{1}=s$ e $\operatorname{grade} G(\mathfrak{F})_{+} \geq s-1$. Então $\operatorname{reg} F(\mathfrak{F}) \geq \operatorname{reg} G(\mathfrak{F})$. Além disso, se depth $F(\mathfrak{F}) \geq s-1$ então

$$
\operatorname{reg} F(\mathfrak{F})=\operatorname{reg} G(\mathfrak{F})
$$

Demonstração. Se $s=1$, pelo Teorema 3.3, o resultado é verdadeiro. Então podemos supor $s \geq 2$. Pela Proposição 3.7, existem geradores $x_{1}, \ldots, x_{s}$ de uma redução minimal $J$ de $I_{1}$ tal que $x_{1}^{*}, \ldots, x_{s}^{*} \in I_{1} / I_{2}$ é uma sequência superficial para $G(\mathfrak{F})$ e $x_{1}^{o}, \ldots, x_{s}^{o} \in I_{1} / \mathfrak{m} I_{1}$ é uma sequência superficial para $F(\mathfrak{F})$. Por hipótese, temos que grade $G(\mathfrak{F})_{+} \geq s-1 . \operatorname{Logo} x_{1}^{*}, \ldots, x_{s-1}^{*}$ é $G(\mathfrak{F})$ regular devido a Proposição 1.13. Denotamos $\overline{\mathfrak{F}}=\frac{\mathfrak{F}+\left(x_{1}, \ldots, x_{s-1}\right)}{\left(x_{1}, \ldots, x_{s-1}\right)}$. Por [HZ, Lema 3.4], temos

$$
G(\overline{\mathfrak{F}}) \simeq G(\mathfrak{F}) /\left(x_{1}^{*}, \ldots, x_{s-1}^{*}\right)
$$

e pelo Lema 3.8,

$$
F(\overline{\mathfrak{F}}) \simeq F(\mathfrak{F}) /\left(x_{1}^{o}, \ldots, x_{s-1}^{o}\right)
$$

Como $x_{1}^{*}, \ldots, x_{s-1}^{*}$ é uma sequência regular, $\operatorname{reg} G(\overline{\mathfrak{F}})=\operatorname{reg} G(\mathfrak{F})$. Pelo Lema 3.9, $\operatorname{dim} F(\overline{\mathfrak{F}})=$ $\operatorname{dim} F(\mathfrak{F})-(s-1)=1$. Usando [HM, Proposição 3.5] e [BH, Proposição 1.2.10(d)], temos

$$
\operatorname{grade} \frac{I_{1}}{\left(x_{1}, \ldots, x_{s-1}\right)}=\operatorname{grade} I_{1}-(s-1)=1
$$

Logo, pelo Teorema 3.3, obtemos $\operatorname{reg} F(\overline{\mathfrak{F}})=\operatorname{reg} G(\overline{\mathfrak{F}})=\operatorname{reg} G(\mathfrak{F})$. Por [BS, Proposição 18.3.11], $\operatorname{reg} F(\overline{\mathfrak{F}}) \leq \operatorname{reg} F(\mathfrak{F})$, de modo que

$$
\operatorname{reg} G(\mathfrak{F}) \leq \operatorname{reg} F(\mathfrak{F})
$$

Se em adição depth $F(\mathfrak{F}) \geq s-1$, o resultado segue do Teorema 3.5. 
O corolário abaixo pode ser obtido como um caso particular do Teorema 3.10.

Corolário 3.11. Seja $\mathfrak{F}$ uma boa filtração equimúltipla onde $s=s(\mathfrak{F})$. Se $G(\mathfrak{F})$ é CohenMacaulay então $\operatorname{reg} G(\mathfrak{F}) \leq \operatorname{reg} F(\mathfrak{F})$. Se além disso, $\operatorname{depth} F(\mathfrak{F}) \geq s-1$ então $\operatorname{reg} F(\mathfrak{F})=$ $\operatorname{reg} G(\mathfrak{F})=a_{s}(G(\mathfrak{F}))+s$.

Demonstração. Use [HZ, Proposição 3.6 e Teorema 3.8].

Corolário 3.12. Seja $(R, \mathfrak{m})$ um anel Noetheriano local onde I é um ideal $\mathfrak{m}$-primário de multiplicidade mista minimal. Se G(I) é Cohen-Macaulay, então F(I) é Cohen-Macaulay se e somente se $\operatorname{reg} F(I) \leq 1$.

Demonstração. Segue por [JPV, Proposição 3.1].

Corolário 3.13. Seja $(R, \mathfrak{m})$ um anel Noetheriano local, onde I é um ideal equimúltiplo. Se $G(I)$ é Cohen-Macaulay, então F(I) é Cohen-Macaulay com multiplicidade minimal se e somente se $\operatorname{reg} F(I) \leq 1$.

Demonstração. Segue por [Vi, Corolário 3.10].

Exemplo 3.14. Um ideal de Sally, considerado em [JPV], é um ideal m-primário em um anel local $(R, \mathfrak{m})$ de Cohen-Macaulay tal que $\ell\left(I^{2} / J I\right)=1$ para qualquer redução minimal $J$. Para um tal ideal, podemos obter por [Ro, Corolário 1.7], que reg $F(I) \geq \operatorname{reg} G(I)$.

Proposição 3.15. Seja $(R, \mathfrak{m})$ um anel local Noetheriano e $\mathfrak{F}$ uma boa filtração. Se reg $R(\mathscr{F}) \leq$ $\operatorname{reg} R(\mathfrak{F})$, então

$$
\operatorname{reg} F(\mathfrak{F})=\operatorname{reg} G(\mathfrak{F})
$$

Demonstração. Aplicando a Proposição 1.21(3) à sequência exata (3.1), obtemos

$$
\operatorname{reg} \mathfrak{m} G(\mathfrak{F})(-1)=\operatorname{reg} \mathfrak{m} G(\mathfrak{F})+1 \leq \max \{\operatorname{reg} R(\mathfrak{F})-1, \operatorname{reg} R(\mathscr{F})\}
$$

Mas por hipótese, $\operatorname{reg} R(\mathscr{F}) \leq \operatorname{reg} R(\mathfrak{F})$ de modo que $\operatorname{reg} \mathfrak{m} G(\mathfrak{F})+1 \leq \operatorname{reg} R(\mathfrak{F})$. Agora novamente aplicando a Proposição 1.21(3) à sequência exata (3.2) e usando o fato que $\operatorname{reg} R(\mathfrak{F})=$ $\operatorname{reg} G(\mathfrak{F})$ (Corolário 2.4), temos que

$$
\operatorname{reg} F(\mathfrak{F}) \leq \max \{\operatorname{reg} \mathfrak{m} G(\mathfrak{F})-1, \operatorname{reg} G(\mathfrak{F})\} \leq \operatorname{reg} R(\mathfrak{F})
$$


Para a outra desigualdade, primeiramente apliquemos a Proposição 1.21(2) à sequência exata (3.2), e desde que $\operatorname{reg} \mathfrak{m} G(\mathfrak{F}) \leq \operatorname{reg} R(\mathfrak{F})-1$, temos

$$
\operatorname{reg} R(\mathfrak{F}) \leq \max \{\operatorname{reg} \mathfrak{m} G(\mathfrak{F}), \operatorname{reg} F(\mathfrak{F})\}=\operatorname{reg} F(\mathfrak{F})
$$

Proposição 3.16. Seja $(R, \mathfrak{m})$ um anel Noetheriano local e $\mathfrak{F}$ uma boa fitração, onde $s:=s(\mathfrak{F})$. Se $R(\mathscr{F})$ é um $R(\mathfrak{F})$-módulo de Cohen-Macaulay, então

$$
\operatorname{reg} F(\mathfrak{F})=\operatorname{reg} G(\mathfrak{F})
$$

Demonstração. Por [RS1, Proposição 4.2], temos que $\operatorname{dim}_{R(\mathfrak{F})} R(\mathscr{F}) \geq \operatorname{dim}_{R} R(\mathscr{F})$. Desde que $\operatorname{dim}_{R} R(\mathscr{F})=\operatorname{dim} R\left(\operatorname{ver}\left[\mathrm{RS} 2\right.\right.$, Lema 1.2.2]), segue que $\operatorname{depth}_{R(\mathfrak{F})} R(\mathscr{F}) \geq s$. Então $H^{i}(R(\mathscr{F}))=$ 0 para $i<s$, e pelo Lema 3.1, obtemos $\operatorname{reg} R(\mathscr{F}) \leq \operatorname{reg} R(\mathfrak{F})$. O resultado seguirá então da Proposição 3.15 .

O próximo resultado foi primeiramente encontrado por Jayanthan e Nanduri em [JN] para o caso $I$-adico, onde $I$ é um ideal m-primário. Abaixo, temos mostrado que esse resultado é de fato válido para qualquer ideal $I$ e também pode ser estendido ao caso de qualquer boa filtração.

Temos assumido desde o início do capítulo que $I_{n} \subseteq \mathfrak{m} I_{n-1}$, para $n \geq 0$. Em particular, se a igualdade é verdadeira para $n \gg 0$, podemos encontrar o seguinte:

Proposição 3.17. Seja $(R, \mathfrak{m})$ um anel local Noetheriano tal que grade $I_{1}>0$. Suponha que $I_{n}=\mathfrak{m} I_{n-1}$ para $n \gg 0$. Então

$$
\operatorname{reg} F(\mathfrak{F})=\operatorname{reg} G(\mathfrak{F})
$$

Demonstração. Existe um $n_{0}$ tal que $I_{n}=\mathfrak{m} I_{n-1}$ para $n \geq n_{0}$. É fácil mostrar que $R(\mathfrak{F})_{+}^{n_{0}} \mathfrak{m} G(\mathfrak{F})=$ 0 . Assim, desde que $\mathfrak{m} G(\mathfrak{F})$ é uma $R(\mathfrak{F})_{+}$-torsão,

$$
H_{R(\mathfrak{F})_{+}}^{i}(\mathfrak{m} G(\mathfrak{F}))=0
$$

para $i>0 \mathrm{e}$

$$
H_{R(\mathfrak{F})_{+}}^{0}(\mathfrak{m} G(\mathfrak{F}))=\mathfrak{m} G(\mathfrak{F})
$$


Se $\mathfrak{m} G(\mathfrak{F})=0$, temos que $\mathfrak{m} I_{n}=I_{n+1}$ para todo $n$. Logo $F(\mathfrak{F})=G(\mathfrak{F})$ e assim, a proposição segue trivialmente. Assuma que $\mathfrak{m} G(\mathfrak{F}) \neq 0$. Pela sequência exata (3.2), obtemos que

$$
0 \rightarrow H^{0}(\mathfrak{m} G(\mathfrak{F})) \rightarrow H^{0}(G(\mathfrak{F})) \rightarrow H^{0}(F(\mathfrak{F})) \rightarrow 0
$$

e $H^{i}(G(\mathfrak{F})) \cong H^{i}(F(\mathfrak{F}))$ para $i>0$. Assim $a_{0}(F(\mathfrak{F})) \leq a_{0}(G(\mathfrak{F}))$ e $a_{i}(G(\mathfrak{F}))=a_{i}(F(\mathfrak{F}))$ para $i>0$. Note que grade $G(\mathfrak{F})_{+}$é igual a zero. De fato, se grade $G(\mathfrak{F})_{+}>0$, pelo Teorema A.20, $H^{0}(G(\mathfrak{F}))=0$. Então, pela sequência exata acima, $H^{0}(\mathfrak{m} G(\mathfrak{F}))=0$, o que é uma contradição. Por hipótese e [HZ, Proposição 3.5],

$$
a_{0}(F(\mathfrak{F})) \leq a_{0}(G(\mathfrak{F}))<a_{1}(G(\mathfrak{F}))=a_{1}(F(\mathfrak{F}))
$$

Portanto

$$
\operatorname{reg} F(\mathfrak{F})=\max \left\{a_{i}(F(\mathfrak{F}))+i: i \geq 1\right\}=\max \left\{a_{i}(G(\mathfrak{F}))+i: i \geq 1\right\}=\operatorname{reg} G(\mathfrak{F})
$$

Proposição 3.18. Sejam $(R, \mathfrak{m})$ um anel local Noetheriano e $\mathfrak{F}$ uma boa filtração tal que $s:=$ $s(\mathfrak{F})$. Suponhamos que $\mathfrak{m} G(\mathfrak{F})$ seja um $R(\mathfrak{F})$-módulo de Cohen-Macaulay de dimensão s. Então

(1) a filtração $\mathfrak{F}$ é na verdade de Hilbert;

(2) $\operatorname{reg} F(\mathfrak{F}) \leq \operatorname{reg} R(\mathfrak{F})$;

(3) se $a_{s}(R(\mathscr{F}))-1<a_{s}(R(\mathfrak{F}))$ então $\operatorname{reg} F(\mathfrak{F})=\operatorname{reg} R(\mathfrak{F})$;

(4) se $a_{s}(R(\mathscr{F}))-1=a_{s}(R(\mathfrak{F}))$ então $\operatorname{reg} \mathfrak{m} G(\mathfrak{F}) \leq \operatorname{reg} R(\mathfrak{F})$ e $\operatorname{reg} F(\mathfrak{F}) \leq \operatorname{reg} R(\mathfrak{F})$. Além disso, se $\operatorname{reg} \mathfrak{m} G(\mathfrak{F})<\operatorname{reg} G(\mathfrak{F})$ então $\operatorname{reg} F(\mathfrak{F})=\operatorname{reg} R(\mathfrak{F})$.

Demonstração. Por [RS1, Proposição 4.2], temos que $\operatorname{dim}_{R / I_{1}} \mathfrak{m} G(\mathfrak{F})=0$. Além disso, por [RS2, Corolário 1.2.2], podemos concluir que $\operatorname{dim} R /\left(I_{1}: \mathfrak{m}\right)=0$, de modo que $I_{1}$ é então $\mathfrak{m}$ primário.

Desde que $\mathfrak{m} G(\mathfrak{F})$ é também um módulo de Cohen-Macaulay sobre $G(\mathfrak{F})$ e fazendo uso da 
sequência de Mayer-Vietoris graduada (ver Comentário A.21), obtemos

$$
H^{i}(\mathfrak{m} G(\mathfrak{F}))=0 \text { para } i \neq s
$$

Pela sequência exata (3.1), temos

$$
\ldots \rightarrow H^{i}(R(\mathfrak{F})) \rightarrow H^{i}(R(\mathscr{F})) \rightarrow H^{i}(\mathfrak{m} G(\mathfrak{F}))(-1) \rightarrow H^{i+1}(R(\mathfrak{F})) \rightarrow \ldots
$$

Então $H^{i}(R(\mathfrak{F})) \cong H^{i}(R(\mathscr{F}))$ para $i \neq s$, de modo que $a_{i}(R(\mathscr{F}))=a_{i}(R(\mathfrak{F}))$ para $i \neq s$.

Primeiro provemos (3). Por hipótese, $a_{s}(R(\mathscr{F})) \leq a_{s}(R(\mathfrak{F}))$. Assim $a_{i}(R(\mathscr{F})) \leq a_{i}(R(\mathfrak{F}))$ para todo $i$, de modo que $\operatorname{reg} R(\mathscr{F}) \leq \operatorname{reg} R(\mathfrak{F})$. Pela Proposição 3.15, $\operatorname{reg} F(\mathfrak{F})=\operatorname{reg} R(\mathfrak{F})$.

Agora provemos (4). Se $a_{s}(R(\mathscr{F}))-1=a_{s}(R(\mathfrak{F}))$, temos

$$
\operatorname{reg} R(\mathscr{F}) \leq \operatorname{reg} R(\mathfrak{F})+1
$$

Pela sequência exata (3.1), temos

$$
\begin{aligned}
\operatorname{reg}(\mathfrak{m} G(\mathfrak{F})(-1)) & =\operatorname{reg} \mathfrak{m} G(\mathfrak{F})+1 \\
& \leq \max \{\operatorname{reg} R(\mathfrak{F})-1, \operatorname{reg} R(\mathscr{F})\} \\
& \leq \operatorname{reg} R(\mathfrak{F})+1 .
\end{aligned}
$$

Pela sequência exata (3.2),

$$
\operatorname{reg} F(\mathfrak{F}) \leq \max \{\operatorname{reg} \mathfrak{m} G(\mathfrak{F})-1, \operatorname{reg} G(\mathfrak{F})\} \leq \operatorname{reg} R(\mathfrak{F})
$$

Assim (4) está provada.

Pelo Lema 3.1, $a_{s}(R(\mathscr{F}))-1 \leq a_{s}(R(\mathfrak{F}))$. Então por (3) e (4), temos (2).

Finalmente, assumamos que $\operatorname{reg} \mathfrak{m} G(\mathfrak{F})<\operatorname{reg} G(\mathfrak{F})$. Pela sequência exata (3.2), obtemos

$$
\operatorname{reg} G(\mathfrak{F}) \leq \max \{\operatorname{reg} \mathfrak{m} G(\mathfrak{F}), \operatorname{reg} F(\mathfrak{F})\}=\operatorname{reg} F(\mathfrak{F})
$$

Assim $\operatorname{reg} R(\mathfrak{F}) \leq \operatorname{reg} F(\mathfrak{F})$. A outra desigualdade é dada pela afirmação (2). 



\section{Capítulo}

\section{A Propriedade da fibra especial ser Gorenstein para o caso de filtrações}

A propriedade do anel graduado associado e da álgebra de Rees serem Gorenstein tem sido bastante estudada, como pode ser visto nos artigos [HRZ], [GN], [HZ], [TVZ], [I], [HKU1] e [HKU2]. Goto e Nishida, em [GN], investigaram a propriedade da álgebra de Rees simbólica ser Cohen-Macaulay e Gorenstein, e então eles generalizaram os resultados obtidos para a álgebra de Rees $R(\mathfrak{F})$ e o anel graduado associado $G(\mathfrak{F})$, onde $\mathfrak{F}$ é uma filtração em geral. Além disso, em [HZ], Zarzuela e Hoa dão algumas consequências que generalizam resultados conhecidos no caso $I$-adico. Por exemplo, eles mostram, sob certas condições, que a propriedade de $R(\mathfrak{F})$ ser Gorenstein implica a propriedade de $G(\mathfrak{F})$ ser Gorenstein. No artigo [HKU1], Heinzer, Kim e Ulrich dão um critério, em termos de ideais, para que o anel graduado $G(I)$ associado a um ideal I seja Gorenstein. Depois eles generalizam este resultado para filtrações de Hilbert, como pode ser visto em [HKU2].

Em [JN], Jayanthan e Nanduri obtém, para o caso de filtrações $I$-adico, uma expressão para o módulo canônico da fibra especial. Eles também dão um critério que caracteriza a propriedade da fibra especial ser Gorenstein. Verificamos que estes resultados permanecem válidos para o caso de boas filtrações $\mathfrak{F}$ tais que $I_{1}$ seja m-primário, ou seja, filtrações de Hilbert. Além disso, temos feito uma nova versão para a caracterização citada, como pode ser vista no Teorema 4.13.

Neste capítulo, assumimos que $(R, \mathfrak{m})$ é um anel local Noetheriano de dimensão $d$ com corpo residual infinito e $\mathfrak{F}$ uma boa filtração. Além disso, sempre supomos que a fibra especial $F(\mathfrak{F})$ e o anel graduado associado $G(\mathfrak{F})$ sejam Cohen-Macaulay. Se a dimensão da fibra especial é 
maior que zero, pelo [CZ, Lema 3.1], $I_{n+1} \subseteq \mathfrak{m} I_{n}$ para todo $n \geq 0$ e então $F(\mathfrak{F})=G(\mathfrak{F}) / \mathfrak{m} G(\mathfrak{F})$. Mesmo quando $F(\mathfrak{F})$ tiver dimensão nula, assumiremos que esta inclusão seja verdadeira. Isso é requerido na maior parte dos resultados deste capítulo tais como a proposição a seguir.

\subsection{Descrição do módulo canônico da fibra especial}

Veremos nesta seção algumas decrições do módulo canônico da fibra especial e do anel graduado associado. Além disso, mostramos que os $a$-invariantes da fibra especial e do anel graduado associado são iguais, como pode ser visto na proposição abaixo.

Proposição 4.1. Seja $(R, \mathfrak{m})$ um anel local Noetheriano de dimensão d e $\mathfrak{F}$ uma filtração de Hilbert tal que o anel graduado associado $G(\mathfrak{F})$ é Cohen-Macaulay. Seja $\omega_{G(\mathfrak{F})}=\oplus_{n \in \mathbb{Z}} \omega_{n}$ e $\omega_{F(\mathfrak{F})}$ o módulo canônico de $G(\mathfrak{F})$ e $F(\mathfrak{F})$, respectivamente. Então

(1) $\omega_{F(\mathfrak{F})} \cong \oplus_{n \in \mathbb{Z}}\left(0: \omega_{n} \mathfrak{m}\right)$;

(2) $a(F(\mathfrak{F}))=a(G(\mathfrak{F}))=r_{J}(\mathfrak{F})-d$, onde $r:=r_{J}(\mathfrak{F})$ é o número de redução de $\mathfrak{F}$ com respeito a qualquer redução minimal $J \subseteq I_{1}$;

(3) $a\left(F\left(\mathfrak{F}^{(k)}\right)\right)=\left[\frac{a(F(\mathfrak{F}))}{k}\right]=\left[\frac{r-d}{k}\right]$ (onde $[y]$ denota o maior inteiro que não seja maior que y), para qualquer $k \in \mathbb{N}$.

(4) $\operatorname{Se} G(\mathfrak{F})$ é Gorenstein então

$$
\omega_{F(\mathfrak{F})} \cong \bigoplus_{n \in \mathbb{Z}} \frac{\left(I_{n+r-d+1}: \mathfrak{m}\right) \cap I_{n+r-d}}{I_{n+r-d+1}}
$$

Demonstração. Primeiramente, observe por [HZ, Proposição 3.6 e Teorema 3.8] que os $a$ invariantes de $G(\mathfrak{F})$, definidos com suporte no ideal irrelevante $G(\mathfrak{F})_{+}$e no ideal maximal homogêneo $\mathfrak{M}$ de $G(\mathfrak{F})$, são iguais.

(1) Adaptando a prova de [M, Teorema 15.7], pode-se mostrar que $\operatorname{dim} G(\mathfrak{F})=d$. Desde que $I_{1}$ é um ideal m-primário, $\operatorname{dim} F\left(I_{1}\right)=d$. Por [HZ, Lema 2.8], temos $\operatorname{dim} G(\mathfrak{F})=\operatorname{dim} F(\mathfrak{F})=d$. Por hipótese, $G(\mathfrak{F})$ é Cohen-Macaulay, e assim, usando [HIO, Corolário 36.14], temos

$$
\omega_{F(\mathfrak{F})} \cong \operatorname{Hom}_{G(\mathfrak{F})}\left(F(\mathfrak{F}), \omega_{G(\mathfrak{F})}\right)
$$


É fácil mostrar que

$$
\operatorname{Hom}_{G(\mathfrak{F})}\left(F(\mathfrak{F}), \omega_{G(\mathfrak{F})}\right) \cong\left(0: \omega_{G(\mathfrak{F})} \mathfrak{m} G(\mathfrak{F})\right)=\left(0: \omega_{G(\mathfrak{F})} \mathfrak{m}\right)=\oplus_{n \in \mathbb{Z}}\left(0: \omega_{n} \mathfrak{m}\right)
$$

O resultado segue.

(2) Pela definição $1.20, a(F(\mathfrak{F}))=-\min \left\{n \mid\left[\omega_{F(\mathfrak{F})}\right]_{n} \neq 0\right\}$. Desde que $R / I_{1}$ é Artiniano e $\omega_{n}$ é um $R / I_{1}$-módulo finito, segue que $\omega_{n}$ é um módulo Artiniano. Por [ILL, Teorema A.33], $\omega_{n}$ é uma $\mathfrak{m} / I_{1}$-torsão e por [ILL, Exemplo A.9],

$$
\left(0: \omega_{n} \mathfrak{m}\right)=\left(0: \omega_{n} \mathfrak{m} / I_{1}\right) \subseteq \omega_{n}
$$

é uma extensão essential. Logo

$$
\left(0: \omega_{n} \mathfrak{m}\right) \neq 0 \text { se e somente se } \omega_{n} \neq 0
$$

Portanto $a(F(\mathfrak{F}))=a(G(\mathfrak{F}))$, por (1).

Através de [BH, Teorema 4.5.7], temos que $R$ é Cohen-Macaulay. Note também que $\mathfrak{F}$ é equimúltipla já que $I_{1}$ é m-primário. Desde que $G(\mathfrak{F})$ é Cohen-Macaulay, grade $G(\mathfrak{F})_{+}=$ ht $\left(G(\mathfrak{F})_{+}\right)$. Por [HZ, Lema 2.4], grade $G(\mathfrak{F})_{+}=d$. Agora, as hipótese de [HZ, Proposição 3.6] estão satisfeitas e então a segunda igualdade segue.

(3) Por [HZ, Corolário 4.6], $G\left(\mathfrak{F}^{(k)}\right)$ é Cohen-Macaulay e por [HZ, Teorema 4.4], $a\left(G\left(\mathfrak{F}^{(k)}\right)\right)=$ $\left[\frac{a(G(\mathfrak{F}))}{k}\right]$. Aplicando (2) à filtração Veroneseana $\mathfrak{F}^{(k)}$, temos que

$$
a\left(F\left(\mathfrak{F}^{(k)}\right)\right)=a\left(G\left(\mathfrak{F}^{(k)}\right)\right)=\left[\frac{a(G(\mathfrak{F}))}{k}\right]=\left[\frac{a(F(\mathfrak{F}))}{k}\right]=\left[\frac{r-d}{k}\right]
$$

(4) Suponhamos que $G(\mathfrak{F})$ é Gorenstein. Por [HIO, 36.13], temos

$$
\omega_{G(\mathfrak{F})} \cong G(\mathfrak{F})(a(G(\mathfrak{F})))=G(\mathfrak{F})(r-d)
$$


Ou seja, $\omega_{n}=I_{n+r-d} / I_{n+r-d+1}$ para todo $n$. Então usando (1), temos

$$
\begin{aligned}
\omega_{F(\mathfrak{F})} \cong \oplus_{n \in \mathbb{Z}}\left(0: \omega_{n} \mathfrak{m}\right) & =\bigoplus_{n \in \mathbb{Z}} \frac{\left(I_{n+r-d+1}: \mathfrak{m}\right) \cap I_{n+r-d}}{I_{n+r-d+1}} \\
& =\bigoplus_{n \geq d-r} \frac{\left(I_{n+r-d+1}: \mathfrak{m}\right) \cap I_{n+r-d}}{I_{n+r-d+1}}
\end{aligned}
$$

Corolário 4.2. Suponha que R tenha dimensão maior ou igual a 2. Se R(F) é Gorenstein então

$$
\omega_{F(\mathfrak{F})} \cong \bigoplus_{n \in \mathbb{Z}} \frac{\left(I_{n-1}: \mathfrak{m}\right) \cap I_{n-2}}{I_{n-1}}
$$

Ou seja, o módulo canônico $\omega_{F(\mathfrak{F})}$ não depende da dimensão d do anel base e do número de redução $r$.

Demonstração. Use [HZ, Corolário 3.11].

No capítulo 3, vimos que se o anel graduado associado $G(\mathfrak{F})$ e a fibra especial $F(\mathfrak{F})$ são Cohen-Macaulay, onde $\mathfrak{F}$ é qualquer filtração de Hilbert, então suas regularidades são iguais. Além disso, vemos abaixo que a regularidade de seus módulos canônicos $\omega_{G(\mathfrak{F})}$ e $\omega_{F(\mathfrak{F})}$ são iguais. Se em adição $G(\mathfrak{F})$ ou $F(\mathfrak{F})$ é Gorenstein, então a regularidade de seus módulos canônicos são iguais à dimensão do anel base.

Corolário 4.3. Se os anéis $G(\mathfrak{F})$ e $F(\mathfrak{F})$ são Cohen-Macaulay temos que $\operatorname{reg} \omega_{G(\mathfrak{F})}=\operatorname{reg} \omega_{F(\mathfrak{F})}$. Além disso, se $G(\mathfrak{F})$ ou $F(\mathfrak{F})$ é Gorenstein temos $\operatorname{reg} \omega_{G(\mathfrak{F})}=\operatorname{reg} \omega_{F(\mathfrak{F})}=d$.

Demonstração. O caso $d=0$ segue de alguns argumentos na prova para o caso de dimensão positiva, como pode ser facilmente verificado pelo leitor. Assumimos então que $d>0$. Pela Proposição 3.7, conseguimos uma redução minimal $J$ de $\mathfrak{F}$ minimamente gerada por $x_{1}, \ldots, x_{d}$ e tal que $x_{1}^{*}, \ldots, x_{d}^{*} \in G(\mathfrak{F})$ e $x_{1}^{o}, \ldots, x_{d}^{o} \in F(\mathfrak{F})$ sejam sequências regulares. Então

$$
F(\mathfrak{F}) /\left(x_{1}^{o}, \ldots, x_{d}^{o}\right) \cong F\left(\mathfrak{F} /\left(x_{1}, \ldots, x_{d}\right)\right) \text { e } G(\mathfrak{F}) /\left(x_{1}^{*}, \ldots, x_{d}^{*}\right) \cong G\left(\mathfrak{F} /\left(x_{1}, \ldots, x_{d}\right)\right)
$$


Por [BH, Corolário 3.6.14],

$$
\operatorname{reg} \omega_{F(\mathfrak{F})}=\operatorname{reg}\left(\omega_{F(\mathfrak{F})} / J^{o} \omega_{F(\mathfrak{F})}\right)=\operatorname{reg} \omega_{F(\mathfrak{F} / J)}+d
$$

e analogamente para $G(\mathfrak{F})$.

Desde que $\operatorname{dim} F(\mathfrak{F} / J)=0=\operatorname{dim} G(\mathfrak{F} / J)$, obtemos $\operatorname{reg} \omega_{F(\mathfrak{F} / J)}=a\left(\omega_{F(\mathfrak{F} / J)}\right)$ e $\operatorname{reg} \omega_{G(\mathfrak{F} / J)}=$ $a\left(\omega_{G(\mathfrak{F} / J)}\right)$.

Aplicando a Proposição 4.1(1) à filtração quociente $\mathfrak{F} / J$, temos

$$
\omega_{F(\mathfrak{F} / J)} \cong \oplus_{n \in \mathbb{Z}}\left(0:\left[\omega_{G(\mathfrak{F} / J)}\right]_{n} \mathfrak{m}\right)
$$

Então por (4.1), temos que $\left[\omega_{F(\mathfrak{F} / J)}\right]_{n} \neq 0 \Leftrightarrow\left[\omega_{G(\mathfrak{F} / J)}\right]_{n} \neq 0$ para todo $n$. Assim $a\left(\omega_{F(\mathfrak{F} / J)}\right)=$ $a\left(\omega_{G(\mathfrak{F} / J)}\right)$, de forma que $\operatorname{reg} \omega_{G(\mathfrak{F})}=\operatorname{reg} \omega_{F(\mathfrak{F})}$.

Além disso, se $G(\mathfrak{F})$ é Gorenstein, por [BH, Proposição 3.6.11], $\omega_{G(\mathfrak{F})} \cong G(\mathfrak{F})(r-d)$. Então

$$
\operatorname{reg} \omega_{G(\mathfrak{F})}=\operatorname{reg} G(\mathfrak{F})(r-d)=\operatorname{reg} G(\mathfrak{F})-r+d
$$

Desde que $G(\mathfrak{F})$ é Cohen-Macaulay, por [HZ, Proposição 3.6], reg $G(\mathfrak{F})=r$, e assim conseguimos que reg $\omega_{G(\mathfrak{F})}=d$. Fazendo o mesmo procedimento para o caso em que $F(\mathfrak{F})$ seja Gorenstein, o resultado segue.

No corolário abaixo, vemos outras descrições (em termos de ideais quocientes) do módulo canônico da fibra especial e do módulo canônico do anel graduado associado.

Corolário 4.4. Sejam $(R, \mathfrak{m})$ um anel local Noetheriano de Gorenstein com dimensão $d>0$ e $\mathfrak{F}$ uma filtração de Hilbert. Suponha que $G(\mathfrak{F})$ é Cohen-Macaulay. Então

$$
\omega_{G(\mathfrak{F})}=\bigoplus_{n \in \mathbb{Z}} \frac{\left(J^{n+r-d}: I_{r}\right)}{\left(J^{n+r-d+1}: I_{r}\right)} \text { e } \omega_{F(\mathfrak{F})}=\bigoplus_{n \in \mathbb{Z}} \frac{\left(J^{n+r-d+1}: \mathfrak{m} I_{r}\right) \cap\left(J^{n+r-d}: I_{r}\right)}{\left(J^{n+r-d+1}: I_{r}\right)}
$$

Além disso, $F(\mathfrak{F})$ é Gorenstein se e somente se

$$
\frac{\left(J^{n+1}: \mathfrak{m} I_{r}\right) \cap\left(J^{n}: I_{r}\right)}{\left(J^{n+1}: I_{r}\right)} \cong \frac{I_{n}}{\mathfrak{m} I_{n}}
$$


para qualquer $n \geq 0$.

Demonstração. Devido as hipóteses deste Corolário, as hipóteses de [HKU2, Teorema 6.1] são também satisfeitas, e então

$$
\omega_{R^{\prime}(\mathfrak{F})}=\bigoplus_{n \in \mathbb{Z}}\left(J^{n+r}: I_{r}\right) t^{n+d-1}
$$

onde $R^{\prime}(\mathfrak{F})$ é a álgebra de Rees estendida. Por [BH, Corolário 3.6.14], têm-se que

$$
\omega_{G(\mathfrak{F})}=\omega_{R^{\prime}(\mathfrak{F}) /\left(t^{-1}\right) R^{\prime}(\mathfrak{F})} \cong\left(\omega_{R^{\prime}(\mathfrak{F})} / t^{-1} \omega_{R^{\prime}(\mathfrak{F})}\right)(-1)
$$

Assim, verifica-se que $\omega_{G(\mathfrak{F})}=\bigoplus_{n \in \mathbb{Z}} \frac{\left(J^{n+r-d}: I_{r}\right)}{\left(J^{n+r-d+1}: I_{r}\right)}$. Depois usamos a Proposição 4.1(1) para obter

$$
\omega_{F(\mathfrak{F})}=\bigoplus_{n \in \mathbb{Z}}\left(0:\left[\omega_{G(\mathfrak{F})}\right]_{n} \mathfrak{m}\right)=\bigoplus_{n \in \mathbb{Z}} \frac{\left(J^{n+r-d+1}: \mathfrak{m} I_{r}\right) \cap\left(J^{n+r-d}: I_{r}\right)}{\left(J^{n+r-d+1}: I_{r}\right)}
$$

Agora supomos que $G(\mathfrak{F})$ seja Gorenstein. Então

$$
\omega_{F(\mathfrak{F})} \cong F(\mathfrak{F})(r-d)=\bigoplus_{n \in \mathbb{Z}} \frac{I_{n+r-d}}{\mathfrak{m} I_{n+r-d}}
$$

Comparando ambos os isomorfismos graduados acima, temos

$$
\frac{\left(J^{n+1}: \mathfrak{m} I_{r}\right) \cap\left(J^{n}: I_{r}\right)}{\left(J^{n+1}: I_{r}\right)} \cong \frac{I_{n}}{\mathfrak{m} I_{n}}
$$

para $n \geq 0$. A recíproca segue facilmente.

\subsection{A propriedade da fibra especial de dimensão um ser Cohen- Macaulay}

Daremos nesta seção, alguns resultados sobre a propriedade da fibra especial ser um anel de Cohen-Macaulay para o caso de boas filtrações. Eles serão necessários na próxima seção, onde daremos uma nova versão para a caracterização da propriedade da fibra especial ser Gorenstein. Além disso, veremos que sob certas condições, a regularidade da fibra especial $F(\mathfrak{F})$ é o número de redução de uma boa filtração equimúltipla $\mathfrak{F}$. 
Lema 4.5. Suponha que $s=\operatorname{dim} F(\mathfrak{F})=1$ e seja $J=(a)$ uma redução minimal de uma boa filtração $\mathfrak{F}$. Assuma que $I_{1}$ contenha um elemento regular. Então para todo n e também $0 \leq i \leq$ $n-1$, temos que

$$
\ell\left(I_{n} /\left(\mathfrak{m} I_{n}+a^{n-i} I_{i}\right)\right)=\mu\left(I_{n}\right)-\mu\left(I_{i}\right)+\ell\left(\left(a^{n-i} I_{i} \cap \mathfrak{m} I_{n}\right) / a^{n-i} \mathfrak{m} I_{i}\right)
$$

Em particular,

$$
\ell\left(I_{n} /\left(\mathfrak{m} I_{n}+a I_{n-1}\right)\right)=\mu\left(I_{n}\right)-\mu\left(I_{n-1}\right)+\ell\left(\left(a I_{n-1} \cap \mathfrak{m} I_{n}\right) / a \mathfrak{m} I_{n-1}\right) .
$$

Demonstração. A hipótese sobre $I_{1}$ dá que $a$ é regular. A sequência exata

$$
0 \rightarrow\left(a^{n}\right) /\left(\mathfrak{m} I_{n} \cap\left(a^{n}\right)\right) \rightarrow I_{n} / \mathfrak{m} I_{n} \rightarrow I_{n} /\left(\mathfrak{m} I_{n}+\left(a^{n}\right)\right) \rightarrow 0
$$

e a igualdade $\mathfrak{m} I_{n} \cap\left(a^{n}\right)=a^{n} \mathfrak{m}$ (onde $J$ é um ideal analiticamente independente sobre $\mathfrak{F}$ ) dão que $\ell\left(I_{n} /\left(\mathfrak{m} I_{n}+\left(a^{n}\right)\right)\right)=\mu\left(I_{n}\right)-\mu\left(J^{n}\right)=\mu\left(I_{n}\right)-1$. Logo o resultado é verdadeiro para $i=0$. Agora seja $1 \leq i \leq n-1$. Então temos as seguintes sequências exatas

$$
\begin{gathered}
0 \rightarrow a^{n-i} I_{i} /\left(a^{n-i} I_{i} \cap \mathfrak{m} I_{n}\right) \rightarrow I_{n} / \mathfrak{m} I_{n} \rightarrow I_{n} /\left(\mathfrak{m} I_{n}+a^{n-i} I_{i}\right) \rightarrow 0 \\
0 \rightarrow\left(a^{n-i} I_{i} \cap \mathfrak{m} I_{n}\right) / a^{n-i} \mathfrak{m} I_{i} \rightarrow a^{n-i} I_{i} / a^{n-i} \mathfrak{m} I_{i} \rightarrow a^{n-i} I_{i} /\left(a^{n-i} I_{i} \cap \mathfrak{m} I_{n}\right) \rightarrow 0 .
\end{gathered}
$$

Também temos o isomorfismo

$$
a^{n-i} I_{i} / a^{n-i} \mathfrak{m} I_{i} \cong I_{i} / \mathfrak{m} I_{i}
$$

desde que $a$ é um elemento regular. O resultado segue fazendo uso da aditividade de $\ell$.

Notação 4.6. Se $a_{1}, \ldots, a_{k} \in R$ e $\mathfrak{F}$ é uma boa filtração, denotamos por

$$
I_{n}^{(k)}=\left(I_{n}+\left(a_{1}, \ldots, a_{k}\right)\right) /\left(a_{1}, \ldots, a_{k}\right)
$$

o n-ésimo termo da filtração $\mathfrak{F} /\left(a_{1}, \ldots, a_{k}\right)$, e o anel quotiente $R /\left(a_{1}, \ldots, a_{k-1}\right)$ por $R_{k-1}$.

O próximo lema mostra como obter o número minimal de geradores de $I_{n}^{(k)}$ em termos do 
número minimal de geradores dos termos da filtração $\mathfrak{F}$.

Lema 4.7. Se $a_{1}, \ldots, a_{k} \in I_{1}$ são tais que $a_{1}^{*}, \ldots, a_{k}^{*} \in G(\mathfrak{F})$ e $a_{1}^{o}, \ldots, a_{k}^{o} \in F(\mathfrak{F})$ sejam sequências regulares, então

$$
\mu\left(I_{n}^{(k)}\right)=\sum_{i=0}^{k}(-1)^{i}\left(\begin{array}{l}
k \\
i
\end{array}\right) \mu\left(I_{n-i}\right) .
$$

Demonstração. A prova é feita por indução sobre $k$. É fácil obter que $\left(a_{1}\right) \cap I_{n}=a_{1} I_{n-1}$ para todo $n \geq 0$. Se $k=1$, pelo teorema dos isomorfimos,

$$
\mu\left(I_{n}^{(1)}\right)=\ell\left(\left(I_{n}+\left(a_{1}\right)\right) /\left(\mathfrak{m} I_{n}+\left(a_{1}\right)\right)=\ell\left(I_{n} /\left(\mathfrak{m} I_{n}+a_{1} I_{n-1}\right)\right) .\right.
$$

Consideremos a seguinte sequência exata natural

$$
0 \rightarrow a_{1} I_{n-1} /\left(a_{1} I_{n-1} \cap \mathfrak{m} I_{n}\right) \rightarrow I_{n} / \mathfrak{m} I_{n} \rightarrow I_{n} /\left(\mathfrak{m} I_{n}+a_{1} I_{n-1}\right) \rightarrow 0
$$

Por [CZ, Proposição 2.3(iii) e Teorema 2.8], temos $a_{1} I_{n-1} \cap \mathfrak{m} I_{n}=a_{1} \mathfrak{m} I_{n-1}$. Além disso, por [HM, Proposição 3.5], $a_{1}$ é um elemento regular. Então $a_{1} I_{n-1} / a_{1} \mathfrak{m} I_{n-1} \cong I_{n-1} / \mathfrak{m} I_{n-1}$ e através da sequência exata acima, temos $\mu\left(I_{n}^{(1)}\right)=\mu\left(I_{n}\right)-\mu\left(I_{n-1}\right)$.

Agora supomos $k>1$. Assim $\bar{a}_{k} \in R_{k-1},\left(\bar{a}_{k}\right)^{*} \in G\left(\mathfrak{F} /\left(a_{1}, \ldots, a_{k-1}\right)\right)$ e $\left(\bar{a}_{k}\right)^{o} \in F\left(\mathfrak{F} /\left(a_{1}, \ldots, a_{k-1}\right)\right)$ são elementos regulares. Já sabemos que a igualdade $\mu\left(I_{n}^{(k)}\right)=\mu\left(I_{n}^{(k-1)}\right)-\mu\left(I_{n-1}^{(k-1)}\right)$ é verdadeira para $k=1$. Então por indução sobre $k$,

$$
\begin{aligned}
\mu\left(I_{n}^{(k)}\right) & =\sum_{i=0}^{k-1}(-1)^{i}\left(\begin{array}{c}
k-1 \\
i
\end{array}\right) \mu\left(I_{n-i}\right)-\sum_{i=0}^{k-1}(-1)^{i}\left(\begin{array}{c}
k-1 \\
i
\end{array}\right) \mu\left(I_{n-1-i}\right) \\
& =\sum_{i=0}^{k}(-1)^{i}\left(\left(\begin{array}{c}
k-1 \\
i
\end{array}\right)+\left(\begin{array}{c}
k-1 \\
i-1
\end{array}\right)\right) \mu\left(I_{n-i}\right) \\
& =\sum_{i=0}^{k}(-1)^{i}\left(\begin{array}{c}
k \\
i
\end{array}\right) \mu\left(I_{n-i}\right)
\end{aligned}
$$

O próximo lema dá, para o caso de boas filtrações, várias caracterizações sobre a propriedade da fibra especial 1-dimensional ser um anel de Cohen-Macaulay. Este lema será essencial na demonstração da Proposição 4.10, onde será usado indução sobre a extensão analítica da boa filtração fixada. 
Lema 4.8. Sejam $(R, \mathfrak{m})$ um anel local Noetheriano, $\mathfrak{F}$ uma boa filtração tal que $I_{1}$ contenha um elemento regular, onde $\operatorname{dim} F(\mathfrak{F})=1$ e ré seu número de redução. Então temos as seguintes condições equivalentes:

(1) $F(\mathfrak{F})$ é Cohen-Macaulay;

(2) $\left(0:_{F(\mathfrak{F})} a^{o}\right)=0$, para qualquer $J=(a) \subseteq I_{1}$ redução minimal de $\mathfrak{F}$;

(3) Para qualquer redução minimal (a) de $\mathfrak{F}, I_{n} \cap\left(\mathfrak{m} I_{n+1}: a\right)=\mathfrak{m} I_{n}, 1 \leq n \leq r-1$;

(4) Para qualquer redução minimal (a) de $\mathfrak{F}, a I_{n} \cap \mathfrak{m} I_{n+1}=a \mathfrak{m} I_{n}$, onde $1 \leq n \leq r-1$;

(5) Para qualquer redução minimal (a) de $\mathfrak{F}, \ell\left(I_{n} /\left(\mathfrak{m} I_{n}+a I_{n-1}\right)\right)=\mu\left(I_{n}\right)-\mu\left(I_{n-1}\right)$, onde $1 \leq n \leq r$.

Demonstração. As equivalências (1) $\Leftrightarrow(2),(2) \Leftrightarrow(3)$ são fáceis de serem encontradas. As sentenças (3) e (4) são equivalentes pelo fato de $a$ ser regular em $R$, enquanto que a equivalência (4) $\Leftrightarrow$ (5) é dada pelo Lema 4.5.

Lema 4.9. Seja $(R, \mathfrak{m})$ um anel local Noetheriano e $\mathfrak{F}$ uma boa filtração. Suponha que $J=$ $\left(a_{1} \ldots, a_{d}\right) R$ é tal que $a_{1}, \ldots, a_{d}$ são partes de um conjunto minimal de geradores para $I_{1} e$ $a_{1}^{*}, \ldots, a_{d-1}^{*}$ seja uma sequência $G(\mathfrak{F})$-regular. Se $J I_{n}+\left(a_{1}, \ldots a_{i}\right) R=I_{n+1}+\left(a_{1}, \ldots a_{i}\right) R$ para $1 \leq i \leq d-1$, então $J I_{n}=I_{n+1}$.

Demonstração. Usando [HZ, Lema 3.4] e indução sobre $i$, é suficiente provar o caso $i=1$. Se $J I_{n}+a_{1} R=I_{n+1}+a_{1} R$ e $z \in I_{n+1}$, então $z=w+a_{1} x$, onde $w \in J I_{n}$ e $x \in R$. Logo $a_{1} x=$ $z-w \in I_{n+1}$, de modo que $x \in\left(I_{n+1}: a_{1}\right)$. Desde que $a_{1}^{*} \in G(\mathfrak{F})$ é regular, temos $\left(I_{n+1}: a_{1}\right)=I_{n}$. Portanto $x \in I_{n}$, e então $z \in J I_{n}$.

Em [CZ3], Cortadellas e Zarzuela mostram sob algumas hipóteses, que a regularidade da fibra especial $F(I)$ é igual ao número de redução de uma redução minimal $J$ do ideal $I$. Aqui verificamos o mesmo resultado para o caso de boas filtrações equimúltiplas. Ressaltamos que a afirmação (1) pode ser facilmente obtida através do Teorema 3.10. 
Proposição 4.10. Seja $(R, \mathfrak{m})$ um anel local Noetheriano e $\mathfrak{F}$ uma boa filtração equimúltipla. Denote $s=\operatorname{dim} F(\mathfrak{F})$ e assuma $\operatorname{grade} I_{1}=s, \operatorname{grade} G(\mathfrak{F})_{+} \geq s-1 e \operatorname{depth} F(\mathfrak{F}) \geq s-1$. Seja $J \subseteq I_{1}$ uma redução minimal de $\mathfrak{F}$ e denote $r=r_{J}(\mathfrak{F})$, o número de redução de $\mathfrak{F}$ com respeito a J. Então obtemos as seguintes:

(1) $\operatorname{reg} F(\mathfrak{F})=r$;

(2) $F(\mathfrak{F})$ é um anel de Cohen-Macaulay se e somente se

$$
\ell\left(I_{n} /\left(\mathfrak{m} I_{n}+J I_{n-1}\right)\right)=\sum_{i=0}^{s}(-1)^{i}\left(\begin{array}{l}
s \\
i
\end{array}\right) \mu\left(I_{n-i}\right),
$$

para $1 \leq n \leq r(\mathfrak{F})$

Demonstração. Se $s=1$, os resultados seguem pelos Lemas 3.2 e 4.8. Suponha que $s>1$. Desde que grade $I_{1}=s$, podemos encontrar uma redução minimal $J=\left(a_{1}, \ldots, a_{s}\right)$ de $\mathfrak{F}$ tal que $a_{1}, \ldots, a_{s} \in R, a_{1}^{*}, \ldots, a_{s-1}^{*} \in G(\mathfrak{F})$ e $a_{1}^{o}, \ldots, a_{s-1}^{o} \in F(\mathfrak{F})$ sejam uma sequência regular em seus respectivos anéis. Desde que $I_{1} /\left(a_{1}, \ldots, a_{s-1}\right) \subset R /\left(a_{1}, \ldots, a_{s-1}\right)$ contém somente um elemento regular, $\operatorname{dim} F\left(\mathfrak{F} /\left(a_{1}, \ldots, a_{\ell-1}\right)\right)=1$ (ver Lema 3.8) e $r_{J}(\mathfrak{F})=r_{J / J_{s-1}}\left(\mathfrak{F} / J_{s-1}\right)$ (ver Lema acima), podemos concluir que

$$
\operatorname{reg} F(\mathfrak{F})=\operatorname{reg} F(\mathfrak{F}) /\left(a_{1}^{o}, \ldots, a_{s-1}^{o}\right)=\operatorname{reg} F\left(\mathfrak{F} / J_{s-1}\right)=r
$$

Temos feito (1).

Por [BH, Exercício 2.1.28] e o fato que

$$
F\left(\mathfrak{F} /\left(a_{1}, \ldots, a_{s-1}\right)\right) \cong F(\mathfrak{F}) /\left(a_{1}^{o}, \ldots, a_{s-1}^{o}\right),
$$

temos que $F(\mathfrak{F})$ é Cohen-Macaulay se e somente se $F\left(\mathfrak{F} /\left(a_{1}, \ldots, a_{\ell-1}\right)\right)$ é Cohen-Macaulay. Se denotarmos o ideal $\left(a_{1}, \ldots, a_{s-1}\right)$ de $R$ por $J_{s-1}$ e o ideal $\mathfrak{m} / J_{s-1} \subset R / J_{s-1}$ por $\mathfrak{m}_{s-1}$, o Lema 4.8 dá que isto é equivalente a

$$
\ell\left(I_{n}^{(s-1)} /\left(\mathfrak{m}_{s-1} I_{n}^{(s-1)}+a_{s} I_{n-1}^{(s-1)}\right)\right)=\mu\left(I_{n}^{(s-1)}\right)-\mu\left(I_{n-1}^{(s-1)}\right)
$$


para $1 \leq n \leq r$. Pelo teorema dos isomorfismo e [HM, Proposição 3.5], temos

$$
\begin{aligned}
I_{n}^{(s-1)} / \mathfrak{m}_{s-1} I_{n}^{(s-1)}+a_{s} I_{n-1}^{(s-1)} & \cong I_{n} /\left(\mathfrak{m} I_{n}+a_{s} I_{n-1}+I_{n} \cap\left(a_{1}, \ldots, a_{s}\right)\right) \\
& \cong I_{n} /\left(\mathfrak{m} I_{n}+\left(a_{1}, \ldots, a_{s}\right) I_{n-1}\right)
\end{aligned}
$$

Pelo Lema 4.7, $\mu\left(I_{n}^{(s-1)}\right)-\mu\left(I_{n-1}^{(s-1)}\right)=\sum_{i=0}^{s}(-1)^{i}\left(\begin{array}{c}s \\ i\end{array}\right) \mu\left(I_{n-i}\right)$. Então (2) segue.

\subsection{A propriedade da fibra especial ser Gorenstein}

Damos nesta seção um critério para que a fibra especial (com respeito a filtrações de Hilbert) seja um anel de Gorenstein. Além disso, apresentamos uma nova versão desse critério, que foi inicialmente apresentado no caso $I$-adico por Jayanthan e Nanduri em [JN].

Notação 4.11. Pela Proposição 3.7, conseguimos uma redução minimal $J=\left(x_{1}, \ldots, x_{d}\right)$ de $\mathfrak{F}$ tal que $x_{1}^{*}, \ldots, x_{d}^{*} \in G(\mathfrak{F})$ e $x_{1}^{o}, \ldots, x_{d}^{o} \in F(\mathfrak{F})$ sejam sequências superficiais para os seus respectivos anéis. Denotamos $J_{i}=\left(x_{1}, \ldots, x_{i}\right), J_{0}=0$ e $J^{*}=\left(x_{1}^{*}, \ldots, x_{d}^{*}\right), J^{o}=\left(x_{1}^{o}, \ldots, x_{d}^{o}\right)$.

Lema 4.12. Suponha que $G(\mathfrak{F})$ e $F(\mathfrak{F})$ sejam anéis de Cohen-Macaulay. Então $G(\mathfrak{F})$ é Gorenstein se e somente se $G\left(\mathfrak{F} / J_{i}\right)$ é Gorenstein, e $F(\mathfrak{F})$ é Gorenstein se e somente se $F\left(\mathfrak{F} / J_{i}\right)$ é Gorenstein.

Demonstração. Seja $J=\left(x_{1}, \ldots, x_{d}\right)$ uma redução minimal de $\mathfrak{F}$ tal que $x_{1}^{*}, \ldots, x_{d}^{*} \in G(\mathfrak{F})$ e $x_{1}^{o}, \ldots, x_{d}^{o} \in F(\mathfrak{F})$ sejam sequências superficiais. Desde que $G(\mathfrak{F})$ e $F(\mathfrak{F})$ são anéis de CohenMacaulay, essas sequências são também regulares (ver Proposição 1.13). Então para qualquer $i$ tal que $1 \leq i \leq d, G(\mathfrak{F}) /\left(x_{1}^{*}, \ldots, x_{i}^{*}\right) \cong G\left(\mathfrak{F} /\left(x_{1}, \ldots, x_{i}\right)\right)$ e $F(\mathfrak{F}) /\left(x_{1}^{*}, \ldots, x_{i}^{*}\right) \cong F\left(\mathfrak{F} /\left(x_{1}, \ldots, x_{i}\right)\right)$ (ver [HZ, Lema 3.4] e Lema 3.8). Por [BH, Proposição 3.1.19 e Exercício 2.1.28], o resultado segue.

O próximo teorema, que se encontra generalizado para o caso de filtrações de Hilbert, dá uma nova versão do Teorema 3.5 no artigo [JN], onde é dado uma caracterização para a propriedade da fibra especial ser Gorenstein, uma vez que se tenha sob hipótese que o anel graduado associado seja Gorenstein. 
Teorema 4.13. Sejam $(R, \mathfrak{m})$ um anel local Noetheriano e $J$ uma redução minimal de uma filtração de Hilbert $\mathfrak{F}$ com número de redução r e extensão analítica s. Suponha que $G(\mathfrak{F})$ seja Gorenstein e $F(\mathfrak{F})$ seja Cohen-Macaulay. Então $F(\mathfrak{F})$ é Gorenstein se e somente se

$$
\ell\left(\frac{\left(\left(I_{n+1}+J\right): \mathfrak{m}\right) \cap I_{n}}{I_{n+1}+J I_{n-1}}\right)=\sum_{i=0}^{s}(-1)^{i}\left(\begin{array}{l}
s \\
i
\end{array}\right) \mu\left(I_{n-i}\right) \text { e } \ell\left(\frac{\left(I_{1}: \mathfrak{m}\right)}{I_{1}}\right)=1
$$

para $1 \leq n \leq r$. Se em adição, supomos que $R / I_{1}$ seja Gorenstein, a segunda igualdade pode ser retirada.

Demonstração. Desde que $G(\mathfrak{F})$ e $F(\mathfrak{F})$ são Cohen-Macaulay, pelas Proposições 3.7 e 1.13, conseguimos uma redução minimal $J$ gerada por elementos cujas imagens correspondentes formam uma sequência regular no anel $G(\mathfrak{F})$ e na fibra especial $F(\mathfrak{F})$. Aplicando a Proposição 4.1 à filtração quociente $\mathfrak{F} / J$ e usando o fato que $\operatorname{dim} F(\mathfrak{F} / J)=0$, pode-se provar que

$$
\omega_{F(\mathfrak{F} / J)}=\bigoplus_{n \in \mathbb{Z}}\left[\frac{\left(\left(I_{n+r+1}+J\right): \mathfrak{m}\right) \cap I_{n+r}+J}{I_{n+r+1}+J}\right]
$$

Pelo teorema dos isomorfimos e o fato que $J \cap I_{n}=J I_{n-1}$ para todo $n$, temos

$$
\left[\omega_{F(\mathfrak{F} / J)}\right]_{n-r} \cong \frac{\left(\left(I_{n+1}+J\right): \mathfrak{m}\right) \cap I_{n}}{I_{n+1}+J I_{n-1}}
$$

Se $F(\mathfrak{F})$ é Gorenstein, pelo Lema 4.12, então $F(\mathfrak{F} / J)$ também é Gorenstein, e dessa forma $\omega_{F(\mathfrak{F} / J)}=F(\mathfrak{F} / J)(r)$. É fácil checar que $[F(\mathfrak{F} / J)(r)]_{n-r}=I_{n} /\left(\mathfrak{m} I_{n}+J I_{n-1}\right)$. Então aplicando $\ell$ em (4.3), teremos

$$
\ell\left(\frac{\left(\left(I_{n+1}+J\right): \mathfrak{m}\right) \cap I_{n}}{I_{n+1}+J I_{n-1}}\right)=\ell\left(\frac{I_{n}}{\mathfrak{m} I_{n}+J I_{n-1}}\right)
$$

para todo $n$. Pela Proposição 4.10, temos o resultado desejado.

Para a recíproca, novamente usamos a Proposição 4.10, obtendo então que a igualdade de comprimentos acima é válida para $0 \leq n \leq r$, e portanto para todo $n$, já que $J$ é uma redução de F. Assim, temos por $(4.3), \ell\left(\left[\omega_{F(\mathfrak{F} / J)}\right]_{n}\right)=\ell\left([F(\mathfrak{F} / J)(r)]_{n}\right)$ para todo $n$, e então $\ell\left(\omega_{F(\mathfrak{F} / J)}\right)=$ $\ell(F(\mathfrak{F} / J)(r))=\ell(F(\mathfrak{F} / J))$. Seja $\mu=\mu\left(\omega_{F(\mathfrak{F} / J)}\right)$ o número minimal de geradores de $\omega_{F(\mathfrak{F} / J)}$. Podemos construir de uma forma natural uma aplicação sobrejetiva $\psi: H \rightarrow \omega_{F(\mathfrak{F} / J)}$ tal que $H$ seja um $F(\mathfrak{F} / J)$-módulo graduado livre de posto $\mu$. Por [HIO, Corolário 36.11], o módulo 
canônico $\omega_{F(\mathfrak{F} / J)}$ tem dimensão injetiva finita e igual a depth $F(\mathfrak{F} / J)=\operatorname{dim} F(\mathfrak{F} / J)=0$. Dessa forma, $\omega_{F(\mathfrak{F} / J)}$ é um módulo injetivo e consequentemente, o funtor $\operatorname{Hom}_{F(\mathfrak{F} / J)}\left(-, \omega_{F(\mathfrak{F} / J)}\right)$ é exato. Então também temos uma aplicação sobrejetiva

$$
\psi^{*}: \operatorname{Hom}_{F(\mathfrak{F} / J)}\left(\omega_{F(\mathfrak{F} / J)}, \omega_{F(\mathfrak{F} / J)}\right) \rightarrow \operatorname{Hom}_{F(\mathfrak{F} / J)}\left(H, \omega_{F(\mathfrak{F} / J)}\right)
$$

Por [BS, Teorema 13.3.4(ii)], temos

$$
\operatorname{Hom}_{F(\mathfrak{F} / J)}\left(\omega_{F(\mathfrak{F} / J)}, \omega_{F(\mathfrak{F} / J)}\right) \cong F(\mathfrak{F} / J)
$$

Naturalmente,

$$
\operatorname{Hom}_{F(\mathfrak{F} / J)}\left(H, \omega_{F(\mathfrak{F} / J)}\right) \cong \bigoplus_{\mu} \omega_{F(\mathfrak{F} / J)}
$$

Temos conseguido uma aplicação sobrejetiva $F(\mathfrak{F} / J) \rightarrow \bigoplus_{\mu} \omega_{F(\mathfrak{F} / J)}$. Assim

$$
\mu \cdot \ell\left(\omega_{F(\mathfrak{F} / J)}\right)=\ell\left(\bigoplus_{\mu} \omega_{F(\mathfrak{F} / J)}\right) \leq \ell(F(\mathfrak{F} / J))
$$

Isto implica que $\mu=1$. Por [BH, Proposição 3.6.11], $F(\mathfrak{F} / J)$ é Gorenstein, e então pelo Lema 4.12 o resultado segue.

Em [BH, Teorema 4.5.7], é provado que se $G(\mathfrak{F})$ é Gorenstein, $R$ também o é. Mas em geral, isso não ocorre para fibras especiais: Em [JPV, Exemplo 6.3], é mostrado que se $R=$ $k\left[\left[t^{4}, t^{5}, t^{6}, t^{7}\right]\right], I=\left(t^{4}, t^{5}, t^{6}\right)$ e $J=\left(t^{4}\right)$ (redução minimal de $I$ ), então $F(I)$ é Gorenstein.

O Corolário abaixo obtém condições suficientes para que $R / I_{1}$ seja Gorenstein.

Corolário 4.14. Seja $(R, \mathfrak{m})$ um anel local Noetheriano e $\mathfrak{F}$ uma filtração de Hilbert. Se ambos o anel $G(\mathfrak{F})$ e a fibra especial $F(\mathfrak{F})$ forem Gorenstein, então também $R / I_{1}$ o é.

Demonstração. Pelo Teorema 4.13, para $n=0$, temos $\ell\left(\left(I_{1}: \mathfrak{m}\right) / I_{1}\right)=1$. Isso implica que $\left(I_{1}: \mathfrak{m}\right) / I_{1} \cong R / \mathfrak{m}$. O resultado está então concluído devido a [ILL, Teorema 11.12].

O próximo resultado mostra que o módulo canônico da fibra especial pode sob certas hipóteses ser um submódulo da mesma. 
Corolário 4.15. Seja $(R, \mathfrak{m})$ um anel local Noetheriano e $\mathfrak{F}$ uma filtração de Hilbert. Assuma que $G(\mathfrak{F})$ seja Gorenstein, $F(\mathfrak{F})$ seja Cohen-Macaulay e $R / I_{1}$ seja Gorenstein. Se supomos que a igualdade $\left(I_{n+r-d+1}: \mathfrak{m}\right) \cap \mathfrak{m} I_{n+r-d}=I_{n+r-d+1}$ é verdadeira para todo $n \geq d-r+1$, obtemos que $\omega_{F(\mathfrak{F})}$ é um submódulo de $F(\mathfrak{F})$.

Demonstração. Desde que $I_{n+1} \subseteq \mathfrak{m} I_{n}$ para todo $n$, existe uma aplicação natural

$$
\psi_{n}: \frac{\left(I_{n+r-d+1}: \mathfrak{m}\right) \cap I_{n+r-d}}{I_{n+r-d+1}} \rightarrow \frac{I_{n+r-d}}{\mathfrak{m} I_{n+r-d}}
$$

para $n \geq d-r$, onde

$$
\operatorname{Ker} \psi_{n}=\frac{\left(I_{n+r-d+1}: \mathfrak{m}\right) \cap \mathfrak{m} I_{n+r-d}}{I_{n+r-d+1}} .
$$

Por (4.2), temos uma aplicação natural $F(\mathfrak{F})$-linear

$$
\psi: \omega_{F(\mathfrak{F})} \rightarrow F(\mathfrak{F})
$$

Desde que $R / I_{1}$ é Gorenstein, e fazendo uso de [ILL, Teorema 11.12], obtemos que $\left(I_{1}: \mathfrak{m}\right) / I_{1} \cong$ $R / \mathfrak{m}$, donde segue que Ker $\psi_{d-r}=0$. Então pela última hipótese,

$$
\operatorname{ker} \psi=\bigoplus_{n \geq d-r} \frac{\left(I_{n+r-d+1}: \mathfrak{m}\right) \cap \mathfrak{m} I_{n+r-d}}{I_{n+r-d+1}}=0
$$

O resultado está então concluído.

Agora veremos a relação entre a multiplicidade do módulo canônico da fibra especial e do anel graduado associado. Em [JN], é mostrado sob algumas hipóteses, que $e_{0}\left(\omega_{F(I)}\right)<e_{0}\left(\omega_{G(I)}\right)$ a menos que $I=\mathfrak{m}$ (onde $e_{0}$ é a multiplicidade de Hilbert de um módulo graduado). Para o caso de filtrações de Hilbert o resultado é similar, como pode ser visto abaixo.

Proposição 4.16. Seja $(R, \mathfrak{m})$ um anel local Noetheriano e $\mathfrak{F}$ uma filtração de Hilbert tal que o anel graduado associado $G(\mathfrak{F})$ e a fibra especial $F(\mathfrak{F})$ sejam anéis de Cohen-Macaulay. Então

$$
e_{0}\left(\omega_{F(\mathfrak{F})}\right) \leq e_{0}\left(\omega_{G(\mathfrak{F})}\right)
$$

Em adição, se $G(\mathfrak{F})$ é Gorenstein, temos a igualdade se e somente se $\mathfrak{F}$ é uma filtração $\mathfrak{m}$-adica. 
Demonstração. A Proposição 4.1 dá que $\omega_{F(\mathfrak{F})} \subseteq \omega_{G(\mathfrak{F})}$. Pela aditividade de $e_{0}$, temos

$$
e_{0}\left(\omega_{F(\mathfrak{F})}\right) \leq e_{0}\left(\omega_{G(\mathfrak{F})}\right)
$$

Devido as propriedades do módulo canônico, podemos encontrar uma redução minimal $J=$ $x_{1}, \ldots, x_{d}$ de $\mathfrak{F}$ tal que $x_{1}^{*}, \ldots, x_{d}^{*} \in G(\mathfrak{F})$ sejam sequências regulares com respeito a $G(\mathfrak{F})$ e $\omega_{G(\mathfrak{F})}$, e também que $x_{1}^{o}, \ldots, x_{d}^{o} \in F(\mathfrak{F})$ sejam sequências regulares com respeito a $F(\mathfrak{F})$ e $\omega_{F(\mathfrak{F})}$ (ver [ILL, Exercício 11.36]). Por [BH, Comentário 4.1.11] e fazendo uso do fato que $\operatorname{dim} F(\mathfrak{F} / J)=0$ e $\operatorname{dim} G(\mathfrak{F} / J)=0$, temos que

$$
e_{0}\left(\omega_{G(\mathfrak{F})}\right)=e_{0}\left(\omega_{G(\mathfrak{F} / J)}\right)=\ell\left(\omega_{G(\mathfrak{F} / J)}\right)
$$

e

$$
e_{0}\left(\omega_{F(\mathfrak{F})}\right)=e_{0}\left(\omega_{F(\mathfrak{F} / J)}\right)=\ell\left(\omega_{F(\mathfrak{F} / J)}\right)
$$

Se $e_{0}\left(\omega_{G(\mathfrak{F})}\right)=e_{0}\left(\omega_{F(\mathfrak{F})}\right)$ então $\ell\left(\omega_{F(\mathfrak{F} / J)}\right)=\ell\left(\omega_{G(\mathfrak{F} / J)}\right)$. Pela Proposição 4.1 , temos $\omega_{F(\mathfrak{F} / J)} \subseteq$ $\omega_{G(\mathfrak{F} / J)}$, e assim $\omega_{F(\mathfrak{F} / J)}=\omega_{G(\mathfrak{F} / J)}$. Desde que $G(\mathfrak{F} / J)$ é Gorenstein e usando o isomorfismo (4.3), obtemos $\left(I_{1}: \mathfrak{m}\right) / I_{1}=R / I_{1}$; donde segue que $I_{1}=\mathfrak{m}$.

A recíproca segue trivialmente, desde que por hipótese, $I_{n+1} \subseteq \mathfrak{m} I_{n}$, e portanto $\mathfrak{F}=\left(\mathfrak{m}^{n}\right)_{n \geq 0}$. 



\section{Capítulo \\ 5 \\ O Teorema de Risler-Teissier para boas \\ filtrações}

As multiplicidades mistas tiveram início em 1957 com o artigo de Bhattacharya [Ba], onde ele descreveu polinômios de Hilbert para dois ideais e os seus coeficientes de maior grau. No entanto, o nome multiplicidades mistas apareceu primeiramente no artigo de Teissier [Te], publicado em 1973, no qual, ele e Risler estudaram, usando elementos superficiais, os polinômios de Hilbert e seus coeficientes, agora para um número finito qualquer de ideais. Teissier mostra em [Te], por exemplo, que em um anel Noetheriano local $(R, \mathfrak{m})$ com ideais $I_{1}, \ldots, I_{k} \mathfrak{m}$-primários, as multiplicidades mistas com respeito a esses ideais são um inteiro positivo. Além disso, ele mostra que essas multiplicidades são iguais a multiplicidade de um ideal gerado por uma sequência superficial com respeito aos ideais citados. Este resultado foi então generalizado para o caso de módulos por Pérez e Roberto Bedregal em [BP], através do estudo da multiplicidade de Buchsbaum-Rim. Outro resultado clássico envolvendo multiplicidades mistas, é o teorema de multiplicidade de Rees, que mostra que o ideal gerado por uma redução conjunta tem a mesma multiplicidade que a multiplicidade mista correspondente, como pode ser visto em [R3]. Além disso, Swanson mostra em [Sw], que se o anel base é formalmente equidimensional, a recíproca do teorema de Rees é também verdadeira. No artigo [JPV], Jayanthan, Puthenpurakal e Verma encontram vários resultados sobre a propriedade da fibra especial ser Gorenstein, nos quais foram usados os teoremas mencionados. Inicialmente pretendiamos generalizar os resultados do artigo [JPV] para o caso de filtrações de ideais mais gerais, no entanto, percebemos que ainda se fazia necessário a generalização (para o caso de filtrações de Hilbert) de resultados clássicos, 
como o Teorema de Rees e de Risler-Teissier citados acima.

O objetivo deste capítulo é definir, para o caso de filtrações de Hilbert, os conceitos básicos e necessários a respeito da teoria envolvendo o teorema de Risler-Teissier descrito acima, de modo que possamos mostrar que ele ainda é verdadeiro neste caso mais geral.

Começamos com o conceito fundamental de elementos superficiais, agora com respeito a filtrações mais gerais.

Definição 5.1. Sejam $(R, \mathfrak{m})$ um anel local e $\mathfrak{F}_{1}=\left(I_{n}^{(1)}\right)_{n \geq 0}, \ldots, \mathfrak{F}_{r}=\left(I_{n}^{(r)}\right)_{n \geq 0}$ filtrações de Hilbert. Um elemento $a \in I_{1}^{(1)}$ é chamado superficial para as filtrações $\mathfrak{F}_{1}, \ldots, \mathfrak{F}_{r} \operatorname{se} \operatorname{dim} R /(a)=$ $\operatorname{dim} R-1$ e para algum inteiro não-negativo $c$ e $n_{1}>c, n_{2} \geq 0, \ldots, n_{r} \geq 0$, tivermos

$$
\left(I_{n_{1}}^{(1)} I_{n_{2}}^{(2)} \cdots I_{n_{r}}^{(r)}: a\right) \cap I_{c}^{(1)} I_{n_{2}}^{(2)} \cdots I_{n_{r}}^{(r)}=I_{n_{1}-1}^{(1)} I_{n_{2}}^{(2)} \cdots I_{n_{r}}^{(r)}
$$

Tendo definido elemento superficial, podemos definir de forma natural uma sequência de elementos superficiais.

Definição 5.2. Uma sequência $a_{1}, \ldots, a_{r} \in R$ é chamada uma sequência superficial para as filtrações $\mathfrak{F}_{1}, \ldots, \mathfrak{F}_{r}$ se $a_{i} \in I_{1}^{(i)}$ para cada $1 \leq i \leq r$ e $\overline{a_{i}} \in R_{i-1}=R /\left(a_{1}, \ldots, a_{i-1}\right)$ é superficial para as filtrações $\mathfrak{F}_{i} /\left(a_{1}, \ldots, a_{i-1}\right), \ldots, \mathfrak{F}_{r} /\left(a_{1}, \ldots, a_{i-1}\right)$.

A existência de elementos superficiais com respeito a uma filtração já foi discutida por Rossi e Valla no artigo [RV].

Teorema 5.3. Se $(R, \mathfrak{m})$ é um anel local tal que o corpo residual $R / \mathfrak{m}$ seja infinito, então elementos superficiais existem.

Demonstração. Segue similarmente a [RV, Teorema 1.2.3(2)].

Agora definiremos as multiplicidades mistas com respeito a várias filtrações de Hilbert.

Seja $(R, \mathfrak{m})$ um anel local de dimensão $d$ e sejam $\mathfrak{F}_{1}, \mathfrak{F}_{2}, \ldots, \mathfrak{F}_{r}$ boas filtrações, onde $\mathfrak{F}_{1}$ é uma filtração de Hilbert. Note que

$$
\mathscr{G}:=G\left(\mathfrak{F}_{1}, \ldots, \mathfrak{F}_{r} ; \mathfrak{F}_{1}\right):=\bigoplus_{n_{1}, \ldots, n_{r} \geq 0} \frac{I_{n_{1}}^{(1)} I_{n_{2}}^{(2)} \cdots I_{n_{r}}^{(r)}}{I_{n_{1}+1}^{(1)} I_{n_{2}}^{(2)} \cdots I_{n_{r}}^{(r)}}
$$


é um $G:=G\left(I_{1}^{(1)}, I_{1}^{(2)}, \ldots, I_{1}^{(r)} ; I_{1}^{(1)}\right)$-módulo finito. Veja que neste caso, $G_{0, \ldots, 0}=R / I_{1}^{(1)}$ é Artiniano. Além disso, $\ell_{G_{0, \ldots, 0}}\left(\mathscr{G}_{n_{1}, \ldots, n_{r}}\right)$ é um polinômio de variáveis $n_{1}, \ldots, n_{r}$ de grau $\operatorname{dim} \mathscr{G}-r=$ $(d+r-1)-r=d-1$ para $n_{1}, \ldots, n_{r}$ suficientemente grandes; ver por exemplo [Ci, (1.8)] . Desde que

$$
\ell\left(\frac{I_{n_{1}}^{(1)} I_{n_{2}}^{(2)} \cdots I_{n_{r}}^{(r)}}{I_{n_{1}+1}^{(1)} I_{n_{2}}^{(2)} \cdots I_{n_{r}}^{(r)}}\right)=\ell\left(R / I_{n_{1}+1}^{(1)} I_{n_{2}}^{(2)} \cdots I_{n_{r}}^{(r)}\right)-\ell\left(R / I_{n_{1}}^{(1)} I_{n_{2}}^{(2)} \cdots I_{n_{r}}^{(r)}\right)
$$

resulta, para $n_{1}, \ldots, n_{r}$ suficientemente grandes, que $\ell\left(R / I_{n_{1}}^{(1)} I_{n_{2}}^{(2)} \cdots I_{n_{r}}^{(r)}\right)$ é um polinômio de $n_{1}, \ldots, n_{r}$ variáveis de grau $d$. O escrevemos na forma

$$
\sum_{|\alpha| \leq d} e_{\alpha}\left(\begin{array}{c}
n_{1}+\alpha_{1} \\
\alpha_{1}
\end{array}\right) \cdots\left(\begin{array}{c}
n_{r}+\alpha_{r} \\
\alpha_{r}
\end{array}\right)
$$

onde $\alpha=\left(\alpha_{1}, \ldots, \alpha_{r}\right)$ e $|\alpha|=\alpha_{1}+\ldots+\alpha_{r}$. Além disso, podemos escrever a sua parte homogênea de grau $d$ na forma

$$
\sum_{\alpha_{1}+\ldots+\alpha_{r}=d} \frac{1}{\alpha_{1} ! \cdots \alpha_{r} !} e\left(\mathfrak{F}_{1}^{\left[\alpha_{1}\right]}, \cdots, \mathfrak{F}_{r}^{\left[\alpha_{r}\right]}\right) n_{1}^{\alpha_{1}} \cdots n_{r}^{\alpha_{r}}
$$

onde $e_{\alpha}=e\left(\mathfrak{F}_{1}^{\left[\alpha_{1}\right]}, \cdots, \mathfrak{F}_{r}^{\left[\alpha_{r}\right]}\right) \in \mathbb{Q}$ e é chamado multiplicidade mista (de filtrações) com respeito $a \mathfrak{F}_{1}, \ldots, \mathfrak{F}_{r}$. O símbolo $e\left(\mathfrak{F}_{1}^{\left[\alpha_{1}\right]}, \cdots, \mathfrak{F}_{r}^{\left[\alpha_{r}\right]}\right)$ é uma abreviação, significando que cada $\mathfrak{F}_{i}$ esta sendo listada $\alpha_{i}$ vezes.

Lema 5.4. (Lema de Artin-Rees para multi filtrações) Sejam $R$ um anel Noetheriano e $\mathfrak{F}_{1}, \ldots, \mathfrak{F}_{k}$ boas filtrações. Seja $I \subset R$ um ideal. Então existem $c_{1}, \ldots, c_{k}$ tais que para $n_{i} \geq c_{i}$, onde $i=$ $1, \ldots, k$, têm-se que

$$
I_{n_{1}}^{(1)} \cdots I_{n_{k}}^{(k)} \cap I=I_{n_{1}-c_{1}}^{(1)} \cdots I_{n_{k}-c_{k}}^{(k)}\left(I_{c_{1}}^{(1)} \cdots I_{c_{k}}^{(k)} \cap I\right) .
$$

Demonstração. Consideremos a multi-álgebra de Rees

$$
\mathscr{R}=R\left[\mathfrak{F}_{1} t_{1}, \ldots, \mathfrak{F}_{k} t_{k}\right]=\bigoplus_{n_{1}, \ldots, n_{k} \geq 0} I_{n_{1}}^{(1)} \cdots I_{n_{k}}^{(k)} t_{1}^{n_{1}} \cdots t_{k}^{n_{k}}
$$

que é um anel Noetheriano, já que por hipótese, as $\mathfrak{F}_{i}$ são boas filtrações (Noetherianas). Agora 
consideramos o ideal

$$
\mathscr{I}=\bigoplus_{n_{1}, \ldots, n_{k} \geq 0}\left(\left(I_{n_{1}}^{(1)} \cdots I_{n_{k}}^{(k)}\right) \cap I\right) t_{1}^{n_{1}} \cdots t_{k}^{n_{k}}
$$

de $\mathscr{R}$, o qual é então finitamente gerado. Podemos escrever o ideal $\mathscr{I}$ como sendo finitamente gerado por elementos homogêneos, ou seja,

$$
\mathscr{I}=\left(a_{\alpha_{1}} t^{\alpha_{1}}, \ldots, a_{\alpha_{s}} t^{\alpha_{s}}\right)
$$

para algum inteiro $s \geq 0$, onde os $\alpha_{i}=\left(\alpha_{1}^{i}, \ldots, \alpha_{k}^{i}\right)$ são multi-índices e $t^{\alpha_{i}}=t_{1}^{\alpha_{1}^{i}} \cdots t_{k}^{\alpha_{k}^{i}}$. Seja $d_{j}:=\max \left\{\alpha_{j}^{i} \mid i=1, . ., s\right\}$ para $1 \leq j \leq k$. Seja $q$ tal que para cada $i$, temos $I_{1}^{(i)} I_{n}^{(i)}=I_{n+1}^{(i)}$ para $n \geq q$. Depois tome $c_{j}:=d_{j}+q$. Os $c_{j}$ são os elementos desejados para a solução do problema.

Lema 5.5. Sejam $(R, \mathfrak{m})$ um anel Noetheriano local e $\mathfrak{F}_{1}, \ldots, \mathfrak{F}_{k}$ filtrações de Hilbert. Sejam $i \in \mathbb{Z}$ e $x \in I_{1}^{(1)}$ tais que para algum $c \in \mathbb{N}$ e quaisquer que sejam $n_{1}, \ldots, n_{k} \in \mathbb{Z}$ suficientemente grandes, tenhamos

$$
\left(I_{n_{1}}^{(1)} \cdots I_{n_{k}}^{(k)}: x^{i}\right) \cap I_{c}^{(1)} I_{n_{2}}^{(2)} \cdots I_{n_{k}}^{(k)}=I_{n_{1}-i}^{(1)} I_{n_{2}}^{(2)} \cdots I_{n_{k}}^{(k)}
$$

Então para $n_{1}, \ldots, n_{k} \gg 0$, temos que

$$
\left(I_{n_{1}}^{(1)} \cdots I_{n_{k}}^{(k)}: x^{i}\right)=\left(0: x^{i}\right)+I_{n_{1}-i}^{(1)} I_{n_{2}}^{(2)} \cdots I_{n_{k}}^{(k)} e\left(0: x^{i}\right) \cap I_{n_{1}}^{(1)} I_{n_{2}}^{(2)} \cdots I_{n_{k}}^{(k)}=0
$$

Demonstração. Pelo lema de Artin-Rees, existem $e_{1}, \ldots, e_{k} \in \mathbb{N}$ tais que para $n_{j} \geq e_{j}$, com $j=$ $1, \ldots, k$, temos

$$
I_{n_{1}}^{(1)} \cdots I_{n_{k}}^{(k)} \cap\left(x^{i}\right) \subseteq x^{i} I_{n_{1}-e_{1}}^{(1)} \cdots I_{n_{k}-e_{k}}^{(k)}
$$

Dessa forma, $\left(I_{n_{1}}^{(1)} \cdots I_{n_{k}}^{(k)}: x^{i}\right) \subseteq\left(0: x^{i}\right)+I_{n_{1}-e_{1}}^{(1)} \cdots I_{n_{k}-e_{k}}^{(k)}$. Desde que $I_{1}^{(1)} \subseteq \sqrt{I_{c}^{(1)} I_{e_{2}}^{(2)} \cdots I_{e_{k}}^{(k)}}=\mathfrak{m}$, existe $m \in \mathbb{Z}^{+}$tal que $\left(I_{1}^{(1)}\right)^{m} \subseteq I_{c}^{(1)} I_{e_{2}}^{(2)} \cdots I_{e_{k}}^{(k)}$. Desde que $\mathfrak{F}_{1}$ é um boa filtração, existe um inteiro $d$ tal que $I_{n}^{(1)} \subseteq\left(I_{1}^{(1)}\right)^{n-d}$ para todo $n$. Tomemos $n^{\prime}$ de forma que $n^{\prime}-d \geq m$. Logo, $I_{n^{\prime}}^{(1)} \subseteq$ 
$\left(I_{1}^{(1)}\right)^{n^{\prime}-d} \subseteq\left(I_{1}^{(1)}\right)^{m}$. Ou seja, $I_{n^{\prime}}^{(1)} \subseteq I_{c}^{(1)} I_{e_{2}}^{(2)} \cdots I_{e_{k}}^{(k)}$. Logo, para $n_{1} \geq n^{\prime}+e_{1}, n_{2} \geq e_{2}, \ldots, n_{k} \geq e_{k}$,

$$
\begin{aligned}
\left(I_{n_{1}}^{(1)} \cdots I_{n_{k}}^{(k)}: x^{i}\right) & \subseteq\left(0: x^{i}\right)+I_{n_{1}-e_{1}}^{(1)} \cdots I_{n_{k}-e_{k}}^{(k)} \\
& \subseteq\left(0: x^{i}\right)+I_{n^{\prime}}^{(1)} I_{n_{2}-e_{2}}^{(2)} \cdots I_{n_{k}-e_{k}}^{(k)} \\
& \subseteq\left(0: x^{i}\right)+I_{c}^{(1)} I_{e_{2}}^{(2)} \cdots I_{e_{k}}^{(k)} I_{n_{2}-e_{2}}^{(2)} \cdots I_{n_{k}-e_{k}}^{(k)} \\
& \subseteq\left(0: x^{i}\right)+I_{c}^{(1)} I_{n_{2}}^{(2)} \cdots I_{n_{k}}^{(k)}
\end{aligned}
$$

Dessa forma, para $n_{1}, \ldots, n_{k} \gg 0$,

$$
\begin{aligned}
\left(I_{n_{1}}^{(1)} \cdots I_{n_{k}}^{(k)}: x^{i}\right) & =\left(I_{n_{1}}^{(1)} \cdots I_{n_{k}}^{(k)}: x^{i}\right) \cap\left(\left(0: x^{i}\right)+I_{c}^{(1)} I_{n_{2}}^{(2)} \cdots I_{n_{k}}^{(k)}\right) \\
& =\left(0: x^{i}\right)+\left(I_{n_{1}}^{(1)} \cdots I_{n_{k}}^{(k)}: x^{i}\right) \cap I_{c}^{(1)} I_{n_{2}}^{(2)} \cdots I_{n_{k}}^{(k)} \\
& =\left(0: x^{i}\right)+I_{n_{1}-i}^{(1)} I_{n_{2}}^{(2)} \cdots I_{n_{k}}^{(k)}
\end{aligned}
$$

Em particular, para $n_{1}, \ldots, n_{k} \gg 0$

$$
\begin{aligned}
\left(0: x^{i}\right) \cap I_{n_{1}}^{(1)} \cdots I_{n_{k}}^{(k)} & \subseteq \bigcap_{n_{1} \gg 0}\left(I_{n_{1}}^{(1)} \cdots I_{n_{k}}^{(k)}: x^{i}\right) \cap I_{c}^{(1)} I_{n_{2}}^{(2)} \cdots I_{n_{k}}^{(k)} \\
& \subseteq \bigcap_{n_{1} \gg 0} I_{n_{1}-i}^{(1)} I_{n_{2}}^{(2)} \cdots I_{n_{k}}^{(k)} \\
& \subseteq \bigcap_{n_{1} \gg 0} I_{n_{1}-i}^{(1)}=0 .
\end{aligned}
$$

Observe no lema acima que as hipóteses são satisfeitas se $i=1$ e $x \in I_{1}^{(1)}$ for um elemento superficial com respeito as filtrações em questão.

Em analogia a teoria envolvendo multiplicidades mistas para vários ideais, a saber, relativo à filtrações adicas, desenvolvemos esta mesma teoria, agora com respeito a filtrações de Hilbert.

Se $a \in I_{1}^{(1)}$ então temos a seguinte sequência exata

$$
\begin{aligned}
& 0 \quad \longrightarrow\left(I_{n_{1}}^{(1)} \cdots I_{n_{r}}^{(r)}: a\right) / I_{n_{1}-1}^{(1)} I_{n_{2}}^{(2)} \cdots I_{n_{r}}^{(r)} \quad \longrightarrow \quad R / I_{n_{1}-1}^{(1)} I_{n_{2}}^{(2)} \cdots I_{n_{r}}^{(r)} \quad \stackrel{\cdot a}{\longrightarrow} \\
& \longrightarrow \quad R / I_{n_{1}}^{(1)} \cdots I_{n_{r}}^{(r)} \quad \longrightarrow R /(a)+I_{n_{1}}^{(1)} I_{n_{2}}^{(2)} \cdots I_{n_{r}}^{(r)} \longrightarrow 0 .
\end{aligned}
$$

Desde que as filtrações são de Hilbert, usando a aditividade do comprimento $\ell$, obtemos

$$
\begin{gathered}
\ell\left(R / I_{n_{1}}^{(1)} \cdots I_{n_{r}}^{(r)}\right)-\ell\left(R / I_{n_{1}-1}^{(1)} \cdots I_{n_{r}}^{(r)}\right)= \\
\ell\left(R /(a)+I_{n_{1}}^{(1)} \cdots I_{n_{r}}^{(r)}\right)-\ell\left(\left(I_{n_{1}}^{(1)} \cdots I_{n_{r}}^{(r)}: a\right) / I_{n_{1}-1}^{(1)} I_{n_{2}}^{(2)} \cdots I_{n_{r}}^{(r)}\right) .
\end{gathered}
$$


O teorema abaixo foi mostrado primeiramente por Teissier em [Te]. Temos então verificado, que este teorema permanece válido quando trabalhamos no caso de filtrações de Hilbert.

Teorema 5.6. (Risler e Teissier) Seja $(R, \mathfrak{m})$ um anel Noetheriano de dimensão d e sejam $\mathfrak{F}_{1}, \ldots, \mathfrak{F}_{k}$ filtrações de Hilbert. Suponhamos que $x \in I_{1}^{(1)}$ não pertença a qualquer ideal primo minimal de R. Supomos que para $n_{1}, \ldots, n_{k} \gg 0$, temos

$$
\left(I_{n_{1}}^{(1)} \cdots I_{n_{k}}^{(k)}: x\right) \cap I_{c}^{(1)} I_{n_{2}}^{(2)} \cdots I_{n_{k}}^{(k)}=I_{n_{1}-1}^{(1)} I_{n_{2}}^{(2)} \cdots I_{n_{k}}^{(k)}
$$

Seja $R^{\prime}=R / x R$. Então para quaisquer $d_{1}, \ldots, d_{k} \in \mathbb{N}$ com $d_{1}+\ldots+d_{k}=d$ e $d_{1}>0$, temos

$$
e_{R}\left(\mathfrak{F}_{1}^{\left[d_{1}\right]}, \ldots, \mathfrak{F}_{k}^{\left[d_{k}\right]}\right)=\left\{\begin{array}{cl}
e_{R^{\prime}}\left(\mathfrak{F}_{1}^{\left[d_{1}-1\right]} R^{\prime}, \ldots, \mathfrak{F}_{k}^{\left[d_{k}\right]} R^{\prime}\right), & \text { se } d>1 \\
\ell(R / x R)-\ell(0: x), & \text { se } d=1
\end{array}\right.
$$

Demonstração. Primeiramente, denotamos $\mathfrak{F}_{i} R^{\prime}=\left(\frac{I_{n_{i}}^{(i)}+(x)}{(x)}\right)_{n_{i} \geq 0}$.

A equação (5.1) nos diz que

$$
\begin{gathered}
\ell\left(R / I_{n_{1}}^{(1)} \cdots I_{n_{r}}^{(r)}\right)-\ell\left(R / I_{n_{1}-1}^{(1)} \cdots I_{n_{r}}^{(r)}\right)= \\
\ell\left(R /(a)+I_{n_{1}}^{(1)} \cdots I_{n_{r}}^{(r)}\right)-\ell\left(\left(I_{n_{1}}^{(1)} \cdots I_{n_{r}}^{(r)}: a\right) / I_{n_{1}-1}^{(1)} I_{n_{2}}^{(2)} \cdots I_{n_{r}}^{(r)}\right) .
\end{gathered}
$$

$\operatorname{Mas}\left(I_{n_{1}}^{(1)} \cdots I_{n_{r}}^{(r)}: a\right)=(0: a)+I_{n_{1}-1}^{(1)} I_{n_{2}}^{(2)} \cdots I_{n_{r}}^{(r)}$ e $(0: a) \cap I_{n_{1}}^{(1)} I_{n_{2}}^{(2)} \cdots I_{n_{r}}^{(r)}=0$ para $n_{1}, \ldots, n_{r} \gg 0$ devido ao Lema 5.5. Portanto, fazendo uso da aditividade de $\ell$ sobre sequências exatas, obtemos

$$
\ell\left(\frac{\left(I_{n_{1}}^{(1)} \cdots I_{n_{r}}^{(r)}: a\right)}{I_{n_{1}-1}^{(1)} I_{n_{2}}^{(2)} \cdots I_{n_{r}}^{(r)}}\right)=\ell(0: a)
$$

Assim, para $n_{1}, \ldots, n_{r} \gg 0$, a parte de maior grau do lado esquerdo da equação resultante de (5.1) é

$$
\sum_{d_{1}+\ldots+d_{k}=d} \frac{1}{d_{1} ! \cdots d_{r} !} e\left(\mathfrak{F}_{1}^{\left[d_{1}\right]}, \cdots, \mathfrak{F}_{k}^{\left[d_{k}\right]}\right)\left(n_{1}^{d_{1}}-\left(n_{1}-1\right)^{d_{1}}\right) n_{2}^{d_{2}} \cdots n_{k}^{d_{k}}
$$

A parte de maior grau na soma acima é

$$
\sum_{d_{1}+\ldots+d_{k}=d} \frac{1}{\left(d_{1}-1\right) ! \cdots d_{r} !} e\left(\mathfrak{F}_{1}^{\left[d_{1}\right]}, \cdots, \mathfrak{F}_{k}^{\left[d_{k}\right]}\right) n_{1}^{d_{1}-1} n_{2}^{d_{2}} \cdots n_{k}^{d_{k}}
$$


Agora sobre o lado direito, se $d>1$, a parte de maior grau é

$$
\sum_{d_{1}+\ldots+d_{k}=d-1} \frac{1}{d_{1} ! \cdots d_{r} !} e_{R^{\prime}}\left(\mathfrak{F}_{1}^{\left[d_{1}\right]} R^{\prime}, \cdots, \mathfrak{F}_{k}^{\left[d_{k}\right]} R^{\prime}\right) n_{1}^{d_{1}} \cdots n_{k}^{d_{k}}
$$

e se $d=1$, o maior grau do lado direito é a constante $e_{R^{\prime}}\left(\mathfrak{F}_{1}^{[0]} R^{\prime}, \cdots, \mathfrak{F}_{k}^{[0]} R^{\prime}\right)-\ell(0: a)=\ell\left(R^{\prime}\right)-$ $\ell(0: a)$. Logo, se $d_{1}+\ldots+d_{k}=d$ então $\left(d_{1}-1\right)+d_{2} \ldots+d_{k}=d-1$, e portanto o resultado segue.

O lema abaixo é bastante similar ao Lema 5.5, havendo apenas uma diferença na superficialidade do elemento $x$, como pode ser visto pelo leitor.

Lema 5.7. Sejam $(R, \mathfrak{m})$ um anel Noetheriano local e I um ideal $\mathfrak{m}$-primário de $R$. Suponhamos que x seja um não-divisor de zero em $R$ ou que para alguma filtração de Hilbert $\mathscr{F}=\left(J_{n}\right)_{n \geq 0}$, exista um elemento $x \in R$ que seja superficial para $\mathscr{F}$. Então existe $e \in \mathbb{N}$ tal que para todo $n \geq e$,

$$
\left(I^{n}: x\right) \subseteq(0: x)+I^{n-e} \quad e(0: x) \cap I^{e}=0 .
$$

Além disso, se x é superficial para I, então para $n \gg 0$,

$$
\left(I^{n}: x\right)=(0: x)+I^{n-1} .
$$

Demonstração. Pelo Lema de Artin-Rees, existe um inteiro $k \geq 0$ tal que para $n \geq k, I^{n} \cap(x) \subseteq$ $x I^{n-k}$. Assim, $\left(I^{n}: x\right) \subseteq(0: x)+I^{n-k}$. Agora, analisemos os seguintes casos. Se $x$ é um nãodivisor de zero, então $(0: x)=0$. Agora suponhamos que $x$ seja superficial para $\mathscr{F}$. Por hipótese, existe $c$ tal que $\left(J_{n}: x\right) \cap J_{c}=J_{n-1}$ para $n \geq c$. Tome $e=c m+k$. Pela inclusão acima, obtemos que

$$
\left(I^{n}: x\right) \subseteq(0: x)+I^{n-e} .
$$

Além disso, seja $m \in \mathbb{N}$ tal que $I^{m} \subseteq J_{1}$. Então temos

$$
(0: x) \cap I^{e} \subseteq \bigcap_{n \geq c}\left(J_{n}: x\right) \cap J_{c} \subseteq \bigcap_{n \geq c} J_{n-1}=0,
$$

desde que $I^{m c} \subseteq\left(J_{1}\right)^{c} \subseteq J_{c}$. Finalmente, suponhamos agora que $x$ seja superficial para $I$, ou seja, 
que tenhamos $\mathfrak{F}=\left(J_{n}\right)_{n \geq 0}=\left(I^{n}\right)_{n \geq 0}$. Então para $n \geq e+c$,

$$
\begin{aligned}
\left(I^{n}: x\right) & =\left((0: x)+I^{c}\right) \cap\left(I^{n}: x\right) \\
& =(0: x)+I^{c} \cap\left(I^{n}: x\right) \\
& =(0: x)+I^{n-1} .
\end{aligned}
$$

Lema 5.8. Seja $(R, \mathfrak{m})$ um anel Noetheriano local de dimensão d. Suponhamos que I seja um ideal $\mathfrak{m}$-primário de $R$ e $x \in I$. Denotemos $R^{\prime}=R / x R$ e $I^{\prime}=I R^{\prime}$. Então temos que:

(1) Se $\operatorname{dim} R^{\prime}=d-1$, então para $n \gg 0$, temos que $\ell\left(\left(I^{n}: x\right) / I^{n-1}\right)$ é um polinômio em $n$ de grau no máximo d-1 com coeficientes racionais.

(2) Se $\operatorname{dim} R^{\prime}=d-1$, então $e_{R^{\prime}}\left(I^{\prime}\right) \geq e_{R}(I)$, e a igualdade ocorre se e somente se para $n \gg 0$, tivermos que $\ell\left(\left(I^{n}: x\right) / I^{n-1}\right)$ seja um polinômio em $n$ de grau no máximo $d-2$.

Demonstração. Seja $g(n)=\ell\left(\left(I^{n}: x\right) / I^{n-1}\right)$. Através da sequência exata

$$
0 \rightarrow \frac{\left(I^{n}: x\right)}{I^{n-1}} \rightarrow \frac{R}{I^{n-1}} \stackrel{x}{\rightarrow} \frac{R}{I^{n}} \rightarrow \frac{R}{(x)+I^{n}} \rightarrow 0
$$

podemos concluir que

$$
g(n)=\ell\left(\frac{R}{(x)+I^{n}}\right)+\ell\left(\frac{R}{I^{n-1}}\right)-\ell\left(\frac{R}{I^{n}}\right)
$$

Assim, desde que $\operatorname{dim} R^{\prime}=d-1$, para $n$ suficientemente grande, $g(n)$ é um polinômio de grau no máximo $d-1$, visto que além disso as partes de grau $d$ acima se cancelam. Isso também significa em termos de multiplicidade que $e_{R^{\prime}}\left(I^{\prime}, R^{\prime}\right) \geq e_{R}(I, R)$; ocorrendo a igualdade se e somente se $g(n)$ é um polinômio em $n$ de grau no máximo $d-2$.

Lema 5.9. Sejam $R$ um anel Noetheriano e $x_{1}, \ldots, x_{s}$ uma sequência superficial para $\mathfrak{F}$. Então

$$
I_{n} \cap\left(x_{1}, \ldots, x_{s}\right)=\left(x_{1}, \ldots, x_{s}\right) I_{n-1},
$$

para n suficientemente grande. 
Demonstração. Faremos apenas o caso em que $s=1$. O lema seguirá então por indução. Por hipótese, existe um inteiro $c$ tal que $\left(I_{n+1}: x\right) \cap I_{c}=I_{n}$ para $n \geq c$. Pelo Lema 5.4, existe um inteiro $l$ tal que para $n \geq l, I_{n} \cap(x) \subseteq x I_{n-l}$. Tomemos $n>c+l$. Se $y \in I_{n} \cap(x)$, então $y=$ $a x=b x$, onde $a \in\left(I_{n}: x\right)$ e $b \in I_{n-l} \subseteq I_{c}$. Donde temos que $a-b \in(0: x) \subseteq\left(I_{n}: x\right)$ e portanto $b=a-(a-b) \in\left(I_{n}: x\right)$. Provamos então que $I_{n} \cap(x) \subseteq x\left(\left(I_{n}: x\right) \cap I_{c}\right)$. Desde que por hipótese $x$ é um elemento superficial, segue que

$$
I_{n} \cap(x) \subseteq x I_{n-1}
$$

para $n$ suficientemente grande.

Proposição 5.10. Sejam $R$ um anel Noetheriano e $\mathfrak{F}=\left(I_{n}\right)_{n \geq 0}$ uma filtração de Hilbert. Sejam $J \subseteq I_{1}$ um ideal e $x_{1}, \ldots, x_{s} \in J$ uma sequência superficial para $\mathfrak{F}$. Denotemos $L=\left(x_{1}, \ldots, x_{S}\right)$. Assim, se $J(R / L)$ é uma redução da filtração $\left(\left(I_{n}+L\right) / L\right)_{n \geq 0}$, então $J$ é uma redução de $\mathfrak{F}$.

Demonstração. Por hipótese, existe um inteiro $c$ tal que para $n \geq c$,

$$
J\left(\frac{R}{L}\right)\left(\frac{I_{n}+L}{L}\right)=\frac{I_{n+1}+L}{L},
$$

ou seja, $\left(J I_{n}+L\right) / L=\left(I_{n+1}+L\right) / L$. Logo,

$$
I_{n+1} \subseteq\left(J I_{n}+L\right) \cap I_{n+1}=J I_{n}+\left(L \cap I_{n+1}\right) .
$$

Pelo Lema 5.9, para $n$ suficientemente grande, temos que

$$
J I_{n}+\left(L \cap I_{n+1}\right) \subseteq J I_{n}+L I_{n}=J I_{n},
$$

de modo que a prova está assim concluída.

A próxima proposição mostra que quando a dimensão do anel base é maior que um e $x$ é um elemento superficial com respeito a uma filtração, então a multiplicidade do ideal $I$ e do ideal $\frac{I+(x)}{(x)}$ de $R / x R$ são iguais. 
Proposição 5.11. Sejam $(R, \mathfrak{m})$ um anel local Noetheriano de dimensão positiva e I um ideal m-primário. Sejam $x \in I, R^{\prime}=R / x R$ e $I^{\prime}=(I+(x)) /(x)$. Suponhamos que $\operatorname{dim} R^{\prime}=d-1$. Se o ideal I é gerado por d elementos, $x \in I \backslash \mathfrak{m} I$ e além disso, x é um não-divisor de zero ou $x$ é um elemento superficial para alguma filtração $\mathfrak{F}$ de Hilbert, então

$$
e_{R}(I)= \begin{cases}e_{R^{\prime}}\left(I^{\prime}\right) & \text { se } d>1 \\ \ell\left(R^{\prime}\right)-\ell(0: x) & \text { se } d=1\end{cases}
$$

Demonstração. Suponhamos que $x$ seja um elemento superficial para alguma filtração $\mathfrak{F}$ de Hilbert.

Primeiramente, suponhamos que $d=1$. Temos então que $I=(x)$. Desde que $\operatorname{dim} R^{\prime}=0$, a filtração quociente $\left(\left(I_{1}+(x)\right) /(x)\right)^{n}$ é nula para $n \gg 0$. Como $\mathfrak{F}$ é uma boa fitração, existe um inteiro $k$, tal que $I_{n} \subseteq\left(I_{1}\right)^{n-k}$ para todo $n$. Dessa forma, concluímos que $\left(I_{n}+(x)\right) /(x)=0$ para $n \gg 0$. Pelo Lema 5.10, obtemos que $I$ é uma redução de $\mathfrak{F}$. Assim, $e_{R}(I)=e_{R}(\mathfrak{F})$. Desde que por [RV, Proposição 1.3.2(3)], $e(\mathfrak{F})=\ell\left(R^{\prime}\right)-\ell((0: x))$, o resultado segue.

Agora, provemos o caso em que $d>1$. Escrevamos $I=\left(x, x_{2}, \ldots, x_{d}\right)$ e $J=\left(x_{2}, \ldots, x_{d}\right)$. Pelo Lema 5.7, existe $e \in \mathbb{N}$ tal que $(0: x) \cap I^{e}=0$. Como $I=(x)+J$, segue que $\left(I^{n}: x\right)=\left(x I^{n-1}+J^{n}:\right.$ $x)=I^{n-1}+\left(J^{n}: x\right)$. Pelo lema de Artin-Rees, existe $c \in \mathbb{N}$, tal que para $n \geq c, J^{n} \cap(x) \subseteq x J^{n-c}$. Logo, para todo $n \geq c,\left(J^{n}: x\right) \subseteq J^{n-c}+(0: x)$ e assim, $\left(I^{n}: x\right) \subseteq I^{n-1}+J^{n-c}+(0: x)$ de forma que

$$
\frac{\left(I^{n}: x\right)}{I^{n-1}} \subseteq \frac{I^{n-1}+J^{n-c}}{I^{n-1}}+\frac{I^{n-1}+(0: x)}{I^{n-1}}
$$

Pelo Lema 5.7, para $n \gg 0,(0: x) \cap I^{n-1}=0$. Logo, usando a sequência exata

$$
0 \rightarrow(0: x) \cap I^{n-1} \rightarrow(0: x) \rightarrow \frac{(0: x)+I^{n-1}}{I^{n-1}} \rightarrow 0
$$

temos para $n \gg 0$ que

$$
(0: x) \cong \frac{(0: x)+I^{n-1}}{I^{n-1}}
$$

Note agora que $\left(I^{n-1}+J^{n-c}\right) / I^{n-1}$ é um $R / I^{c}$-módulo e assim, é fácil mostrar, que seu comprimento é limitado superiormente por $\mu\left(J^{n-c}\right) \ell\left(R / I^{c}\right)$, o qual é limitado superiormente por $\left(\begin{array}{c}n-c+d-2 \\ d-2\end{array}\right) \ell\left(R / I^{c}\right)$. Este último, é um polinômio sobre a variável $n$ de grau no máximo $d-2$. 
Logo, para o caso $d>1, \ell\left(\left(I^{n}: x\right) / I^{n-1}\right)$ é para $n$ suficientemente grande, um polinômio em $n$ de grau no máximo $d-2$. Pelo Lema 5.8, segue o resultado.

O próximo resultado é uma consequência imediata do Teorema 5.6. É mostrado abaixo que as multiplicidades mistas com respeito a filtrações de Hilbert também são inteiros positivos.

Teorema 5.12. (Risler e Teissier) Sejam $(R, \mathfrak{m})$ um anel local Noetheriano não nulo e $\mathfrak{F}_{1}, \ldots, \mathfrak{F}_{k}$ filtrações de Hilbert. Sejam $d_{1}, . ., d_{k} \in \mathbb{N}$ com $d_{1}+\ldots+d_{k}=d$. Seja $x_{1}, \ldots, x_{d}$ uma sequência superficial para $\mathfrak{F}_{1}, \ldots, \mathfrak{F}_{1}, \ldots, \mathfrak{F}_{k}, \ldots, \mathfrak{F}_{k}\left(\right.$ com cada $\mathfrak{F}_{i}$ listando $d_{i}$ vezes $)$, onde cada $x_{i}$ não pertença a qualquer primo minimal de $\left(x_{1}, \ldots, x_{i-1}\right)$. Então,

$$
e\left(\mathfrak{F}_{1}^{\left[d_{1}\right]}, \ldots, \mathfrak{F}_{k}^{\left[d_{k}\right]}\right)=\ell\left(R /\left(x_{1}, \ldots, x_{d}\right)\right)-\ell\left(\left(x_{1}, \ldots, x_{d-1}: x_{d}\right)\right)
$$

e também igual à multiplicidade do ideal $\left(x_{1}, \ldots, x_{d}\right)$.

Demonstração. Seja $R_{i}=R /\left(x_{1}, \ldots, x_{i}\right)$. Pelo Teorema 5.6,

$$
\begin{aligned}
e_{R}\left(\mathfrak{F}_{1}^{\left[d_{1}\right]}, \ldots, \mathfrak{F}_{k}^{\left[d_{k}\right]}\right) & =e_{R_{1}}\left(\mathfrak{F}_{1}^{\left[d_{1}-1\right]} R_{1}, \ldots, \mathfrak{F}_{k}^{\left[d_{k}\right]} R_{1}\right) \\
& =\ldots \\
& =e_{R_{d-1}}\left(\mathfrak{F}_{1}^{[0]} R_{d-1}, \ldots, \mathfrak{F}_{k-1}^{[0]} R_{d-1}, \mathfrak{F}_{k}^{[1]} R_{d-1}\right) \\
& =e_{R_{d}}\left(\mathfrak{F}_{1}^{[0]} R_{d}, \ldots, \mathfrak{F}_{k}^{[0]} R_{d}\right)-\ell\left(\left(x_{1}, \ldots, x_{d-1}\right): x_{d}\right) \\
& =\ell\left(R /\left(x_{1}, \ldots, x_{d}\right)\right)-\ell\left(\left(x_{1}, \ldots, x_{d-1}\right): x_{d}\right) .
\end{aligned}
$$

Se $d=0$, este número é $\ell(R)$ (que é positivo desde que $R \neq 0$ ). Para o caso $d>0$, basta usarmos a Proposição 5.11 repetidas vezes, para obter que

$$
e_{R}\left(\left(x_{1}, \ldots, x_{d}\right)\right)=\ell\left(R /\left(x_{1}, \ldots, x_{d}\right)\right)-\ell\left(\left(x_{1}, \ldots, x_{d}\right): x_{d}\right)
$$

de forma que temos concluído a demonstração do teorema. 



\section{Apêndice}

\section{Extensão analítica e cohomologia local}

\section{A.1 Extensão analítica}

O objetivo desta tese é estudar a propriedade da fibra especial ser Gorenstein e a sua regularidade de Castelnuovo-Mumford. Dessa forma, é importante introduzir ao leitor algumas informações básicas a respeito da dimensão dessa fibra, chamada de extensão analítica. Começamos por definir a noção de elementos analiticamente independentes sobre uma boa filtração.

Definição A.1. Seja $(R, \mathfrak{m})$ um anel local e $\mathfrak{F}$ uma boa filtração. Então $v_{1}, \ldots, v_{t} \in I_{1}$ são analiticamente independentes para $\mathfrak{F}$ se e somente se, para todo $h \in \mathbb{N}$ e $f \in R\left[X_{1}, \ldots, X_{t}\right]$ (o anel dos polinômios sobre $R$ em $t$ indeterminadas) um polinômio homogêneo de grau $h$ tal que $f\left(v_{1}, \ldots, v_{t}\right) \in I_{h} \mathfrak{m}$, tivermos que todos os coeficientes de $f$ encontram-se no ideal maximal m. Além disso, note que se $v_{1}, \ldots, v_{t} \in I_{1}$ são analiticamente independentes em $\mathfrak{F}$ e também $J=\left(v_{1}, \ldots, v_{t}\right)$, então $J^{h} \cap I_{h} \mathfrak{m}=J^{h} \mathfrak{m}$, para todo $h \in \mathbb{N}$, como é fácil de observar. Quando $\mathfrak{F}=\left(I^{n}\right)_{n \geq 0}$, dizemos que $v_{1}, \ldots, v_{t} \in I$ são analiticamente independentes em $I$.

Lema A.2. Se $(R, \mathfrak{m})$ é um anel local e $J \subseteq I+J \mathfrak{m}$, então $J \subseteq I$.

Demonstração. Use o lema de Nakayama.

Proposição A.3. Seja $(R, \mathfrak{m})$ um anel local tal que $k:=R / \mathfrak{m}$ seja infinito. Se J é uma redução minimal de $\mathfrak{F}$ tal que $I_{1}$ seja um ideal próprio e $v_{1}, \ldots, v_{t}$ formem um conjunto minimal de geradores de J, então $v_{1}, \ldots, v_{t}$ são analiticamente independentes para $\mathfrak{F}$.

Demonstração. Sejam $h \in \mathbb{N}$ e $f \in R\left[X_{1}, \ldots, X_{t}\right]$ um polinômio homogêneo de grau $h$ tal que $f\left(v_{1}, \ldots, v_{t}\right) \in I_{h} \mathfrak{m}$. Mostremos agora que o coeficiente $u$ de $X_{1}$ é uma não unidade de $R$. De fato, 
suponha que $u$ é uma unidade. Então $v_{1}^{h} \in I_{h} \mathfrak{m}+v_{2} J^{h-1}+\ldots+v_{t} J^{h-1}$, de modo que

$$
J^{h}=R v_{1}^{h}+\left(R v_{2}+\ldots+R v_{t}\right) J^{h-1} \subseteq I_{h} \mathfrak{m}+\left(R v_{1}+\ldots+R v_{t}\right) J^{h-1}
$$

Para simplicar, denotamos $J^{\prime}$ por $R v_{2}+\ldots+R v_{t}$. Por hipótese, $J I_{n}=I_{n+1}$ para algum $n$ e então

$$
I_{h+n}=J^{h} I_{n} \subseteq\left(I_{h} \mathfrak{m}+J^{\prime} J^{h-1}\right) I_{n} \subseteq I_{h+n} \mathfrak{m}+J^{\prime} J^{h-1} I_{n}
$$

Pelo Lema acima, $I_{h+n} \subseteq J^{\prime} I_{h+n-1}$, e assim $I_{h+n}=J^{\prime} I_{h+n-1}$. Então $J^{\prime}$ é uma redução de $\mathfrak{F}$, o que é uma contradição, desde que $J^{\prime} \subset J$. Portanto $u \in \mathfrak{m}$.

Agora seja $\left\{r_{i j}\right\}$ uma matrix $t \times t$ sobre $R$, tal que $r_{11}, r_{21}, \ldots, r_{t 1}$ e $\operatorname{det}\left\{r_{i j}\right\}$ sejam unidades de $R$. Dessa forma, existe um conjunto minimal de geradores $\left\{w_{1}, \ldots, w_{t}\right\}$ para $J$ tal que $v_{i}=$ $\sum_{j=1}^{t} r_{i j} w_{j}$, para todo $i=1, \ldots, t$. Desde que

$$
f\left(\sum_{j=1}^{t} r_{1 j} w_{j}, \ldots, \sum_{j=1}^{t} r_{t j} w_{j}\right) \in I_{h} \mathfrak{m}
$$

e $f\left(r_{11}, \ldots, r_{t 1}\right)$ é o coeficiente de $X_{1}^{h}$ na forma $f\left(\sum_{j=1}^{t} r_{1 j} w_{j}, \ldots, \sum_{j=1}^{t} r_{t j} w_{j}\right)$, teremos que $f\left(r_{11}, \ldots, r_{t 1}\right) \in \mathfrak{m}$.

Seja $\bar{r}$ a imagem natural no corpo $k:=R / \mathfrak{m}$ de $r \in R$ e $\bar{q}$ a imagem natural no anel $k\left[X_{1}, \ldots, X_{t}\right]$ de $q \in R\left[X_{1}, \ldots, X_{t}\right]$. Então $\bar{f}\left(\bar{r}_{11}, \ldots \bar{r}_{t 1}\right)=0$. Donde concluímos que $\bar{f}\left(a_{1}, . ., a_{t}\right)=0$, para $\left(a_{1}, . ., a_{t}\right) \in(k \backslash\{0\})^{t}$, desde que $\bar{r}_{11}, \ldots, \bar{r}_{t 1}$ são não nulos e arbitrários em $k:=R / \mathfrak{m}$. Como $k$ é infinito, $\bar{f}=0$, ou seja, todos os coeficientes encontram-se no ideal maximal $\mathfrak{m}$.

Agora iremos definir a extensão analítica de uma filtração $\mathfrak{F}$. Primeiramente definimos a álgebra

$$
F(\mathfrak{F})=\bigoplus_{n \geq 0} \frac{I_{n}}{\mathfrak{m} I_{n}}=R / \mathfrak{m} \oplus I_{1} / I_{2} \oplus \cdots
$$

que é chamada de Fibra Especial de $\mathfrak{F}$. O número $s=s(\mathfrak{F})=\operatorname{dim} F(\mathfrak{F})=\operatorname{dim} R(\mathfrak{F}) / \mathfrak{m} R(\mathfrak{F})($ ver [HZ]) é dito ser a extensão analítica de $\mathfrak{F}$. Quando $\mathfrak{F}$ é a filtração I-adica, a extensão analítica é denotada por $s(I)$. Rees introduziu a noção de reduções básicas de filtrações Noetherianas e mostrou em [R, Teorema 6.12] que $s(\mathfrak{F})$ é igual ao número minimal de geradores de qualquer 
redução minimal. Por [HZ, Lema 2.7], $s(\mathfrak{F})=\operatorname{dim} G(\mathfrak{F}) / \mathfrak{m} G(\mathfrak{F})$ e por [HZ, Lema 2.8], $s(\mathfrak{F})=$ $s\left(I_{1}\right)$.

Comentário A.4. Seja $(R, \mathfrak{m})$ um anel local, $k:=R / \mathfrak{m}$ e $J=\left(v_{1}, \ldots v_{t}\right) \subseteq I \subset R$ ideais tais que $v_{1}, \ldots v_{t}$ sejam analiticamente independentes em $I$. É claro que eles são analiticamente independentes em $J$ e pela Definição A.1, existe um isomorfismo $K\left[X_{1}, \ldots, X_{t}\right] \stackrel{\phi}{\rightarrow} \oplus_{i \geq 0} J^{i} / \mathfrak{m} J^{i} d e$ $k$-álgebras graduadas tal que $\phi\left(X_{i}\right)=v_{i}+\mathfrak{m} J$, para todo $i=1, . ., t$. Logo, $s(J)=t$.

Teorema A.5. Sejam $(R, \mathfrak{m})$ um anel local tal que $k:=R / \mathfrak{m}$ é infinito e seja $\mathfrak{F}$ uma boa filtração tal que $I_{1}$ seja próprio. Se J é uma redução de $\mathfrak{F} e\left\{v_{1}, \ldots, v_{t}\right\}$ é um conjunto minimal de geradores de J, então

(1) $s(\mathfrak{F})=t$;

(2) $J$ é uma redução minimal de $\mathfrak{F}$ se e somente se $s(\mathfrak{F})=t$;

(3) J é uma redução minimal de $\mathfrak{F}$ se e somente se $v_{1}, \ldots, v_{t}$ são analiticamente independentes sobre $\mathfrak{F}$. Em particular, um conjunto de elementos analiticamente independentes sobre $\mathfrak{F}$ é analiticamente independentes em $I_{1}$.

Demonstração. Use o fato que $s(\mathfrak{F})=s\left(I_{1}\right)$, a Proposição A.3 e [BS, Teorema 18.2.4].

\section{A.2 Cohomologia Local}

Nesta seção introduzimos algumas propriedades a respeito de cohomologia local. Alguns resultados serão apenas enunciados. Para um conhecimento mais profundo a respeito desta teoria, referenciamos por exemplo, $[\mathrm{BH}],[\mathrm{BS}]$ ou $[\mathrm{HIO}]$.

Definição A.6. Se $M$ é um $R$-módulo e $I$ um ideal de $R$, tome $\Gamma_{I}(M)=\bigcup_{n \in \mathbb{N}}\left(0: M I^{n}\right)$ como sendo o conjunto de elementos de $M$ aos quais são aniquilados por alguma potência de $I$. É fácil verificar que ele é um submódulo de $M$. Além disso, se $f: M \rightarrow N$ é um homomorfismo de $R$-módulos, existe uma aplicação induzida $\Gamma_{I}(f): \Gamma_{I}(M) \rightarrow \Gamma_{I}(N)$ que está bem definida, já que $f\left(\Gamma_{I}(M)\right) \subseteq \Gamma_{I}(N)$. Se $g: M \rightarrow N$ e $f: N \rightarrow L$ são homomorfismos de $R$-módulos e $r \in R$, então $\Gamma_{I}(h \circ f)=\Gamma_{I}(h) \circ \Gamma_{I}(f), \Gamma_{I}(f+g)=\Gamma_{I}(f)+\Gamma_{I}(g), \Gamma_{I}(r f)=r \Gamma_{I}(f)$ e $\Gamma_{I}\left(I d_{M}\right)=I d_{\Gamma_{I}}(M)$. Logo, $\Gamma_{I}$ é um funtor covariante, chamado funtor I-torsão. 
Sejam $I, J$ ideais de $R$.

Proposição A.7. $\Gamma_{I}\left(\Gamma_{J}(M)\right)=\Gamma_{I+J}(M)$ para qualquer $R$-módulo $M$.

Proposição A.8. $\Gamma_{I}=\Gamma_{J}$ se e somente se $\sqrt{I}=\sqrt{J}$. Logo, se J é uma redução de I, então $\Gamma_{I}=\Gamma_{J}$

Lema A.9. O funtor I-torsão $\Gamma_{I}$ é exato a esquerda.

Demonstração. Se $0 \rightarrow L \stackrel{f}{\rightarrow} M \stackrel{g}{\rightarrow} N \rightarrow 0$ é uma sequência exata de $R$-módulos, precisamos mostrar que

$$
0 \rightarrow \Gamma_{I}(L) \stackrel{\Gamma_{I}(f)}{\rightarrow} \Gamma_{I}(M) \stackrel{\Gamma_{I}(g)}{\rightarrow} \Gamma_{I}(N)
$$

é também exata. Pelos argumentos da Definição A.6 vemos que $\Gamma_{I}(f)$ é um monomorfismo e $\Gamma_{I}(g) \circ \Gamma_{I}(f)=0$. Agora, seja $m \in M$ tal que $I^{n} m=0$ para algum $n \in \mathbb{N}$ e $g(m)=0$. Então, existe $l \in L$ tal que $f(l)=m$. Agora precisamos somente mostrar que $l \in \Gamma_{I}(L)$. Para cada $r \in I^{n}$, temos $f(r l)=r f(l)=r m=0$. Desde que $f$ é um monomorfismo, $r l=0$. Então $I^{n} l=0$.

Exemplo A.10. Considere a sequência exata de $\mathbb{Z}$-módulos $0 \rightarrow 2 \mathbb{Z} \rightarrow \mathbb{Z} \rightarrow \mathbb{Z} / 2 \mathbb{Z} \rightarrow 0$ e mostre que o funtor $2 \mathbb{Z}$-torsão $\Gamma_{2 \mathbb{Z}}$ não é um funtor exato.

Dado um $R$-módulo $M$, seja

$$
I^{\bullet}: 0 \stackrel{d^{-1}}{\rightarrow} I^{0} \stackrel{d^{0}}{\rightarrow} I^{1} \rightarrow \cdots \rightarrow I^{i} \stackrel{d^{i}}{\rightarrow} I^{i+1} \rightarrow \cdots
$$

uma resolução injetiva de $M$. Aplicando o funtor $\Gamma_{I}$ a $I^{\bullet}$, obtemos um novo complexo

$$
0 \stackrel{\Gamma_{I}\left(d^{-1}\right)}{\rightarrow} \Gamma_{I}\left(I^{0}\right) \stackrel{\Gamma_{I}\left(d^{0}\right)}{\rightarrow} \Gamma_{I}\left(I^{1}\right) \rightarrow \cdots \rightarrow \Gamma_{I}\left(I^{i}\right) \stackrel{\Gamma_{I}\left(d^{i}\right)}{\rightarrow} \Gamma_{I}\left(I^{i+1}\right) \rightarrow \cdots
$$

Agora, estamos prontos para definir os funtores (ou módulo) de cohomologia local de $M$ com respeito a um ideal $I$ do anel $R$.

Definição A.11. Para $i \geq 0$, a i-ésima cohomologia local (ou módulo de cohomologia local) com respeito a um ideal $I$, denotada por $H_{I}^{i}(M)$, é definida como sendo

$$
\operatorname{Ker}\left(\Gamma_{I}\left(d^{i}\right)\right) / \operatorname{Im}\left(\Gamma_{I}\left(d^{i-1}\right)\right)
$$


Note que ela é o $i$-ésimo funtor derivado a direta de $\Gamma_{I}$ e que assim, por um argumento de álgebra homológica, isso independe da escolha da resolução injetiva $I^{\bullet}$ do módulo $M$.

Definição A.12. Para um $R$-módulo $M$, chamamos o conjunto $\Gamma_{I}(M)$ de submódulo I-torsão de $M$. Se $\Gamma_{I}(M)=0$, dizemos que $M$ é um $I$-módulo livre de torsão, e se $\Gamma_{I}(M)=M$, dizemos que $M$ é uma I-torsão.

Para ver com mais detalhes a construção da sequência exata longa que é dada abaixo, referenciamos o livro de Brodmann e Sharp [BS].

Comentário A.13. Seja $0 \rightarrow L \rightarrow M \rightarrow N \rightarrow 0$ uma sequência exata de R-módulos. Existe um homomorfismo $H_{I}^{i}(N) \rightarrow H_{I}^{i+1}(L)$, chamado de homomorfismo conectante que gera uma sequência exata longa

$$
\begin{gathered}
0 \longrightarrow H_{I}^{0}(L) \longrightarrow H_{I}^{0}(M) \longrightarrow H_{I}^{0}(N) \longrightarrow H_{I}^{1}(L) \longrightarrow H_{I}^{1}(L) \longrightarrow H_{I}^{1}(N) \\
\longrightarrow \quad \longrightarrow H_{I}^{i}(L) \longrightarrow H_{I}^{i}(M) \longrightarrow H_{I}^{i}(N) \longrightarrow H_{I}^{i+1}(L) \longrightarrow
\end{gathered}
$$

Proposição A.14. Sejam $I, J$ ideais de R tais que $\sqrt{I}=\sqrt{J}$. Então $H_{I}^{i}(M)=H_{J}^{i}(M)$ para cada $R$-módulo $M$ e todo $i \geq 0$.

Demonstração. Mostre que $\Gamma_{I}(M)=\Gamma_{J}(M)$ se e somente se $\sqrt{I}=\sqrt{J}$.

Exemplo A.15. [BS, Exercício 1.2.4] Para todo grupo Abeliano (ou seja, um $\mathbb{Z}$-módulo $G$ ) e para todo $a \in \mathbb{Z}$, temos $H_{a \mathbb{Z}}(G)=0$ para $i \geq 2$.

Exemplo A.16. [BS, Exercício 1.2.5] Suponha que $R$ seja um anel local regular de dimensão $d$. Então $H_{I}^{i}(M)=0$ para qualquer $R$-módulo $M$ e para todo $i>d$.

Outro conceito que será usado neste trabalho é o posto aritmético de um ideal. Isso permite relacionar o número de elementos requeridos para gerar um ideal $I$ com o anulamento da cohomologia local $H_{I}^{i}(M)$ de um módulo $M$.

Definição A.17. O posto aritmético de I, denotado por ara(I), é definido como sendo

$$
\operatorname{ara}(I)=\min \left\{n \geq 0: \text { existam } b_{1}, \ldots, b_{n} \in R \operatorname{com} \sqrt{R b_{1}+\cdots+R b_{n}}=\sqrt{I}\right\} .
$$

Note que o posto aritmético do ideal nulo é zero. 
Definição A.18. Seja $M$ um $R$-módulo não-nulo. A dimensão (de Krull) de $M$, denotada por $\operatorname{dim} M$, é definida como sendo $\operatorname{dim} R / \operatorname{Ann} M$, ou seja, a dimensão de Krull do anel quociente $R /$ Ann $M$, onde Ann $M:=\{x \in R \mid x M=0\}$ é o ideal anulador de $M$.

Assim como no Exemplo A.16, existem diversos e importantes resultados a respeito do anulamento dos módulos de cohomologia local. Por exemplo, se $M$ é um $R$-módulo e $I$ é um ideal de $R$ tal que $I$ contenha uma $M$-sequência de comprimento $n$, então $H_{I}^{i}(M)=0$ para todo $i<n$; ver [BS, Exercício 1.3.9(iv)]. E mais, se $I$ é gerado por $t$ elementos, então para todo $R$-módulo $M$, temos que $H_{I}^{i}(M)=0$ para todo $i>t$; ver [BS, Teorema 3.3.1]. Como consequência, temos que $H_{I}^{i}(M)=0$ para todo $i>$ ara $(I)$, como pode ser visto em [BS, Corolário 3.3.3]. Abaixo, enunciamos o Teorema do Anulamento de Grothendieck que relaciona a dimensão de um módulo e o anulamento de seu módulo de cohomologia local. Para ver a demonstração sugerimos [BS, Teorema 6.1.2].

Teorema A.19. (Teorema do Anulamento de Grothendieck) Se M é um R-módulo e I é um ideal arbitrário do anel $R$, então $H_{I}^{i}(M)=0$, para todo $i>\operatorname{dim} M$.

Existe também um resultado que relaciona o anulamento de módulos de cohomologia local e o comprimento de sequências regulares maximais. Para ver a prova, referenciamos [BS, Teorema 6.2.7]. Para ver o mesmo resultado no caso local sugerimos [BH, Teorema 3.5.7].

Teorema A.20. Se $M$ é um $R$-módulo finitamente gerado tal que IM $\neq M$, então

$$
\operatorname{grade}(I, M)=\inf \left\{i: H_{I}^{i}(M) \neq 0\right\}
$$

A sequência de Mayer-Vietoris, bastante conhecida em topologia algébrica, também possui uma correspondente análoga na teoria de cohomologia local, como pode ser visto no comentário abaixo. Lembrando que a mesma possui sua correspondente no caso de módulos graduados. A contrução desta sequência pode ser encontrada por exemplo, no livro de Brodmann e Sharp [BS], Capítulo 3. Essa sequência é bastante utilizada no presente trabalho, afim de obter resultados sobre a regularidade de Castelnuovo-Mumford da álgebra Rees, do anel graduado associado e da fibra especial. 
Comentário A.21. Seja $R$ um anel (graduado) e $M$ um R-módulo (graduado). Se I e J são ideais (graduados) de R, então existe uma sequência exata longa de módulos de cohomologia local (graduados)

$$
\begin{aligned}
& 0 \longrightarrow H_{I+J}^{0}(M) \longrightarrow H_{I}^{0}(M) \oplus H_{J}^{0}(M) \longrightarrow H_{I \cap J}^{0}(M) \\
& \longrightarrow H_{I+J}^{1}(M) \longrightarrow H_{I}^{1}(M) \oplus H_{J}^{1}(M) \longrightarrow H_{I \cap J}^{1}(M) \\
& \text {... ... } \\
& \longrightarrow H_{I+J}^{i}(M) \longrightarrow H_{I}^{i}(M) \oplus H_{J}^{i}(M) \longrightarrow H_{I \cap J}^{i}(M) \\
& \longrightarrow H_{I+J}^{i+1}(M) \quad \longrightarrow \quad \ldots
\end{aligned}
$$

O próximo resultado é conhecido como o Teorema Básico de Finitude e Anulamento. Este fato generaliza o [BS, Teorema 14.1.4(ii)(iii)], que dá aplicações à geometria projetiva através da cohomologia local de módulos graduados.

Proposição A.22. Se $R=\oplus_{n \geq 0} R_{n}$ é um anel positivamente graduado e $M$ é um $R$-módulo graduado finito, então

(1) para todo $i \geq 0$ e todo $n \in \mathbb{Z}, H_{R_{+}}^{i}(M)_{n}$ é um $R_{0}$-módulo finito;

(2) existe $r \in \mathbb{Z}$ tal que $H_{R_{+}}^{i}(M)_{n}=0$ para todo $i \geq 0$ e todo $n \geq r$.

Para ver a prova do teorema acima, sugerimos ao leitor que veja [BS, Teorema 15.1.5]. 



\section{Referências Bibliográficas}

[A] M. F. Atiyah e I. G. Macdonald, Introduction to commutative algebra, Addison-Wesley Publishing Co., Reading, Mass.-London-Don Mills, Ont. 1969.

[B] N. Bourbaki, Commutative algebra, Addison Wesley, Reading, 1972.

[Ba] P. B. Bhattacharya, The Hilbert function of two ideals, Proc. Cambridge Philos. Soc, 53, $568-575,1957$.

[BH] W. Bruns, J. Herzog, Cohen-Macaulay rings, Revised Edition, Cambridge Studies in Advanced Mathematics, 39. Cambridge University Press, Cambridge, 1998.

[BP] R. C. Bedregal, V. H. Jorge Pérez, Mixed multiplicities and the minimal number of generator of modules, J. Pure Appl. Algebra 214, no. 9, 1642-1653, 2010.

[BS] M. P. Brodmann, R. Y. Sharp, Local cohomology: an algebraic introduction with geometric applications, Cambridge Studies in Advanced Mathematics, 60. Cambridge University Press, Cambridge, 1998.

[C] T. Cortadellas, Fiber cones with almost maximal depth, Comm. Algebra, 33, no. 3, 953$963,2005$.

[Ci] C. Ciupercă, A numerical characterization of the $S_{2}$-ification of a Rees algebra, J. Pure Appl. Algebra 178, no. 1, 25-48, 2003.

[CZ] T. Cortadellas, S. Zarzuela, On the depth of the fiber cone of filtrations, J. Algebra 198, no 2, 428-445, 1997.

[CZ2] T. Cortadellas, S. Zarzuela, Depth formulas for certain graded modules associated to a filtration: a survey, Geometric and combinatorial aspects of commutative algebra (Mes- 
sina, 1999), 145-157, Lecture Notes in Pure and Appl. Math., 217, Dekker, New york, 2001 .

[CZ3] T.Cortadellas, S. Zarzuela, On the scruture of the fiber cone of ideals with analytic spread one, J. Algebra 317, no. 2, 759-785, 2007.

[EG] D. Eisenbud and S. Goto, Linear free resolutions and minimal multiplicity, J. Algebra 88, 1984, 89-133.

[GN] S. Goto, K. Nishida, The Cohen-Macaulay and Gorenstein Rees algebras associated to filtrations, Mem. Amer. Math. Soc. 110, 1994, no. 22, 5635-5656.

[GP] Gert-Martin Greuel, G. Pfister, A Singular introduction to commutative algebra, Second extended edition, Springer, Berlin, 2008.

[H] Hartshorne, Robin, Algebraic geometry, Graduate Texts inMathematics, Springer-Verlag 52, New York-Heidelberg, 1977.

[Hy] E. Hyry, On the Gorenstein property of the associated graded ring of power of a an ideal, Manuscripta Math. 80, 13-20, 1993.

[HIO] M. Herrmann, S. Ikeda, U. Orbanz, Equimultiplicity and blowing up, An algebraic study. With an appendix by B. Moonen. Springer-Verlag, Berlin, 1988.

[HK] W. J. Heinzer, M-K. Kim, Properties of the fiber cone of ideals in local rings, Comm. Algebra 31 (7), 3529-3546, 2003.

[HKU1] W. Heinzer, M. Kim, B. Ulrich, The Gorenstein and complete intersection properties of associated graded rings, J. Pure Appl. Algebra 201, no. 1-3, 264-283, 2005.

[HKU2] W. Heinzer, M. Kim, B. Ulrich, The Cohen-Macaulay and Gorenstein Properties of Rings Associated to Filtrations, Volume 39, Issue 10, 2011.

[HM] S. Huckaba, T. Marley, Hilbert coefficients and the depths of associated graded rings, J. London Math. Soc. (2) 56, no. 1, 64-76, 1997. 
[HRZ] M. Herrmann, J. Ribbe, S. Zarzuela, On the Gorenstein property of Rees and form rings of power of ideals, Trans. Amer. Math. Soc. 342, no. 2, 631-643, 1994.

[HZ] L. T. Hoa and S. Zarzuela, Reduction number and a-invariant of good filtrations, Comm. Algebra 14, no. 22, 5635-5656, 1994.

[I] S. Ikeda, On the Gorensteinness of Rees Algebras over local rings, Nagoya Math. J. 102, 135-154, 1986.

[ILL] S. Iyengar, G. Leuschke, A. Leykin, C. Miller, E. Miller, A. Singh, U. Walther, Twentyfour hours of local cohomology, Graduate Studies in Mathematics, 87. American Mathematical Society, Providence, RI, 2007.

[JN] A. V. Jayanthan, R. Nanduri, Castelnuovo-Mumford regularity and gorensteinness of fiber cone, Comm. Algebra 40, no. 4, 1338-1351, 2012.

[JU] M. Johnson and B. Ulrich, Artin-Nagata properties and Cohen-Macaulay associated graded rings, Compositio Math. 108, 7-29, 1996.

[JPV] A. V. Jayanthan, T. J. Puthenpurakal, J. K. Verma, On fiber cones of $\mathfrak{m}$-primary ideals, Canad. J. Math. 59, no 1, 109-126, 2007.

[JV] A. V. Jayanthan, J. K. Verma, Hilbert coefficients and depth of fiber cones, Journal of Pure and Applied Algebra 201, 97-115, 2005.

[LP] P. H. Lima, V. H. Pérez, Castelnuovo-Mumford regularity of the fiber Cone for good filtrations, Institute of Mathematics and Computer Science, University of São Paulo, 2011.

[M] H. Matsumura, Commutative ring theory, Cambridge University Press, 1980.

[NR] D. G. Northcott e D. Rees, Reductions of ideals in local rings, Proc. Cambridge Philos Soc. 50, 145-158, 1954.

[NV] S. Noh and W. Vasconcelos, The $S_{2}$-closure of a Rees algebra, Results. Math. 23, 149-162, 1993. 
[O] A. Ooishi, Castelnuovo's regularity of graded rings and modules, Hiroshima Math. J. 12, 627-644, 1982.

[P] T. Puthenpurakal, Hilbert-coefficients of a Cohen-Macaulay module, J. Algebra 264, no. 1, 82-97, 2003.

[R] D. Rees, Lectures on the asymptotic theory of ideals, London Math. Soc. Lecture Notes Series 113, Cambridge Univ. Press 1988.

[R2] D. Rees, A-transforms of local rings and a theorem on multiplicities of ideals, Proc. Cambridge Philos. Soc., 57, 8-17, 1961.

[R3] D. Rees, Generalizations of reductions and mixed multiplicities, J. London Math. Soc. 29, 397-414, 1984.

[Ro] M. E. Rossi, A bound on the reduction number of a primary ideal, Proc. Amer. Math. Soc. 128 , no. 5, 1325-1332, 2000.

[RS1] C. Rotthaus, L. M. Sega, Some properties of graded local cohomology modules, J. Algebra 283, no. 1, 232-247, 2005.

[RS2] C. Rotthaus, L. M. Sega, Open loci of graded modules, Trans. Amer. Math. Soc. 358, no. 11, 4959-4980, 2006.

[RR] L. J. Ratliff, Jr., David E. Rush, Two notes on reductions of ideals, Indiana Univ. Math. J. 27, no. 6, 929-934, 1978.

[RV] Maria E. Rossi e Giuseppe Valla, Hilbert Functions of Filtered Modules, Lecture Notes of the Unione Matematica Italiana, Vol. 9., Primeira Ediçao., , XVIII, 100 p, 2010.

[S] Kishor Shah, Coefficient ideals, Trans. Amer. Math. Soc. 327, no. 1, 373-384, 1991.

[Sw] I. Swanson, Mixed multiplicities, Joint reductions, and a theorem of Rees, J. London Math. Soc. 48, 1-14, 1993.

[T] N. V. Trung, The Castelnuovo regularity of the Rees algebra and the associated graded ring, Trans. of Amer. Math. Soc. 350, no. 7, 2813-2832, 1998. 
[Te] B. Teissier, Cycles évanescents, sections planes, et conditions de Whitney, Singularités à Cargèse, Asterisque, Nos. 7 et 8, Paris, Soc. Math. France, p. 285-362, 1973.

[TVZ] N. V. Trung, D. Q. Viêt, S. Zarzuela, When is the Rees algebra Gorenstein ?, J. Algebra 175, 137-156, 1995.

[V] W. V. Vasconcelos, Computational methods in commutative algebra and algebraic geometry, Algorithms and Computation in Mathematics, 2. Springer-Verlag, Berlin, 1998.

[Vi] D. Q. Viêt, On the Cohen-Macaulayness of fiber cones, Proc. Amer. Math. Soc. 136, no. 12, 4185-4195, 2008. 


\section{Índice Remissivo}

*dimensão

de um anel, 8

$a$-invariante, 9

álgebra

de Rees, 4

de Rees simbólica, 35

estendida de Rees, 4

analiticamente independentes

elementos, 63

anel

*local, 7

graduado associado, 4

Bass

número de, 7

boa filtração, 1

Castelnuovo-Mumford

regularidade de, 9

extensão analítica, 64

fecho tight, 4

filter-regular

elemento, 5

sequência, 5

filtração, 1 adica, 2

de Hilbert, 1

Noetheriana, 4

obtida pelo fecho $e_{1}, 3$

funtor

torsão, 65

grade

de um ideal, 68

ideal

irrelevante, 4

maximal, 8

ideal coeficiente, 3

módulo

*canônico, 8

canônico, 7

de cohomologia local, 66

dimensão de Krull de um, 68

livre de torsão, 67

torsão, 67

multi-álgebra

de Rees, 53

multiplicidade

de um módulo graduado, 28

multiplicidades mistas 
de filtrações, 53

posto aritmético, 67

Ratliff-Rush

fecho de, 2

ideal de, 2

redução, 5

minimal, 5

número de, 5

resolução

minimal graduada livre, 13

sequência superficial

para filtrações, 52

superficial

elemento, 5

para filtrações, 52

sequência, 5

Teorema

de Risler-Teissier, 56

Teorema do Anulamento de Grothendieck, 68 